\title{
Length scales in granular matter
}

\author{
Dissertation \\ for the award of the degree \\ "Doctor of Philosophy" Ph.D Division of Mathematics and Natural \\ Sciences \\ of the Georg-August-Universität Göttingen \\ within the doctoral program ProPhys \\ of the Georg-August University School of Science (GAUSS)
}

submitted by

Song-Chuan Zhao

from Shijiazhuang, China

Göttingen, 2013 


\section{Thesis Committee}

Prof. Dr. Stephan Herminghauss, Max-Planck-Institut für Dynamik und Selbstorganisation (MPIDS)

Prof. Dr. Annette Zippelius, Georg-August-Universität

Dr. Matthias Schröter, Max-Planck-Institut für Dynamik und Selbstorganisation (MPIDS)

Members of the Examination Board

Reviewer: Prof. Dr. Stephan Herminghaus

Second Reviewer: Prof. Dr. Annette Zippelius

Further members of the Examination Board:

Dr. Christian Bahr, MPIDS

Prof. Dr. Sara Köster, Georg-August-Universität

Prof. Brian Utter, James Madison University, US

Prof. Dr. Katharina Vollmayr-Lee, Bucknell University, US

Date of the oral examination: 28th February 2013 


\section{Contents}

1 Introduction 1

1.1 Previous studies on length scales . . . . . . . . . . . . . . . . . . . 2

1.2 The existence of correlation between local volumes . . . . . . . . . . . . . . . 3

1.3 Statistical mechanics of granular packings . . . . . . . . . . . . . . . 4

1.4 $\quad$ Dynamic Heterogeneity . . . . . . . . . . . . . . . . . . . . . . . . 5

1.5 Outline of the thesis $\ldots \ldots \ldots \ldots \ldots$. . . . . . . . . . . . . . 7

\begin{tabular}{lll}
\hline I & Packings in 2D & 8
\end{tabular}

\begin{tabular}{|lll}
2 & Experimental Protocol & 9
\end{tabular}

2.1 Two-dimensional bed . . . . . . . . . . . . . . . . . . . . . . . . . . . . 9 9

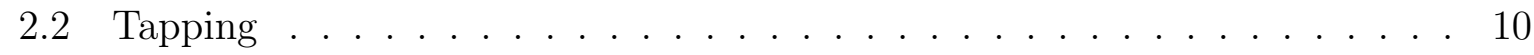

2.3 Illumination and imaging . . . . . . . . . . . . . . . . . . . . . . 11

2.4 Experimental procedure $\ldots \ldots \ldots \ldots \ldots \ldots$. . . . . . . . . . . . . . 12

\begin{tabular}{|lll}
3 & Image Processing & 13
\end{tabular}

3.1 Determining particle centroids . . . . . . . . . . . . . . . . . . . . 13

3.2 Local volume tessellation . . . . . . . . . . . . . . . . . . . . . . . . . . . 15

$3.2 .1 \quad$ Navigation map . . . . . . . . . . . . . . . . . . . . . . . . . . . . . . . . . . . . . . . . . . . . . . . . .

3.2 .2 Radical tessellation . . . . . . . . . . . . . . . . . . . . . 17

\begin{tabular}{|lll}
4 & Correlation between Voronoi volumes in disc packings & 21
\end{tabular}

4.1 Ensuring a steady and homogeneous state . . . . . . . . . . . . . . 21

4.2 The histogram of Voronoi volume and free volume . . . . . . . . . . . . . . 24

4.3 Free volume of a cluster on a grid network . . . . . . . . . . . . . . 26

4.4 Two-point correlation of free Voronoi volume . . . . . . . . . . . . . . . . . 31

4.5 Characteristic lengths of the correlation . . . . . . . . . . . . . . . . 34

4.6 Discussions $\ldots \ldots \ldots \ldots \ldots$. . . . . . . . . . . . . . . . . . . . . . . . 37

4.7 Further results and Outlook $\ldots \ldots \ldots \ldots$

$4.7 .1 \quad$ Radical Tessellation vs. navigation map . . . . . . . . . . . . . . . 39

4.7 .2 Two exponential fit . . . . . . . . . . . . . . . . . . . . 40

$4.7 .3 \quad$ The lengths crossing Random Loose Packing . . . . . . . . . . . . . . 41

5 Configurational temperature $\chi$ in disc packings $\quad 43$

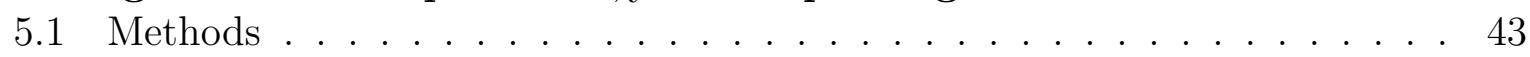

5.2 Measuring the configurational temperature $\chi \ldots \ldots \ldots . \ldots . \ldots . \ldots 45$

$5.2 .1 \chi_{\mathrm{FDT}}$ measured from fluctuation-dissipation theorem . . . . . . . . . 45

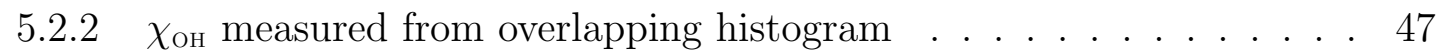

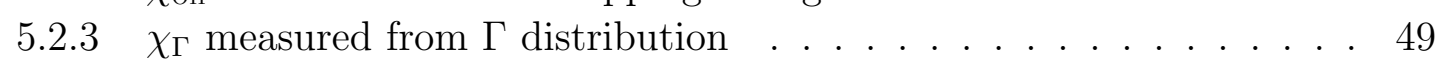

5.3 Discussion . . . . . . . . . . . . . . . . . . . . . . 51

$5.3 .1 \quad$ Entropy $\ldots \ldots \ldots \ldots \ldots \ldots \ldots$

5.3 .2 Information theory and granular statistics . . . . . . . . . . . 53

5.4 Further results . . . . . . . . . . . . . . . . . . 55 
5.4 .1 The finite size effect . . . . . . . . . . . . . . . . . . . . . . . . . 55

5.4 .2 Density of state . . . . . . . . . . . . . . . 58

\begin{tabular}{ll}
\hline Conclusion of Part I & 62
\end{tabular}

$\begin{array}{lll}\text { II } & \text { Packings in 3D } & 63\end{array}$

\begin{tabular}{lll}
\hline 6 & Experimental Protocol & 64
\end{tabular}

6.1 Setup . . . . . . . . . . . . . . . . . . . . . . 64

6.2 Static packings . . . . . . . . . . . . . . . . . . 65

6.3 Dynamic Heterogeneities . . . . . . . . . . . . . . . . 66

\begin{tabular}{lll}
\hline & Image Processing & 68
\end{tabular}

7.1 Binarization . . . . . . . . . . . . . . . . . . . . 68

7.2 Defects removal . . . . . . . . . . . . . . . . . . . . . . . . . . . 71

7.3 Erosion and centroid detection . . . . . . . . . . . . . . . . . . 71

7.4 Convolution correction . . . . . . . . . . . . . . . . . . . . . . . 72

\begin{tabular}{lll}
\hline & Static sphere packings & $\mathbf{7 4}$
\end{tabular}

8.1 Voronoi volume distribution . . . . . . . . . . . . . . . . . . . . . 74

8.2 Correlation between Voronoi volumes . . . . . . . . . . . . . . . . 75

8.3 Compactivity measurement . . . . . . . . . . . . . . . 77

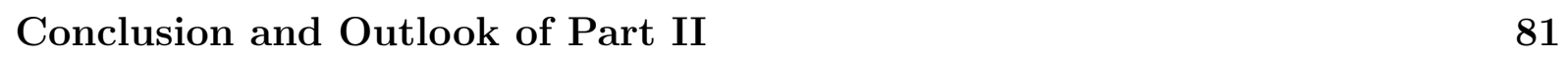

\begin{tabular}{ll}
\hline Bibliography & 82
\end{tabular}

\begin{tabular}{|lr}
\hline Acknowledgements & 87
\end{tabular} 


\section{Chapter 1}

\section{Introduction}

Humans have a long history of dealing with granular materials. Modern industry still devotes a lot of effort to these materials: the construction of highways, levees, and dams depends on the manipulation of large amounts of gravel and sand; bulk commodities from flour and grain to coal are transported processed, and stored. The broad usage of granular materials comes from its various states. At low density sands can fly with wind, making northern China suffer from dust storms every spring in the recent decade; at high density it can be jammed, bearing stress like solid. Even in daily life, e.g., when we fill a salt pot, we can observe the rich behaviours of granular materials - salt powders flow like liquid then settle in a stable pile; shake it and the pile collapses. Fig. 1.1 presents examples of different states of granular matter.
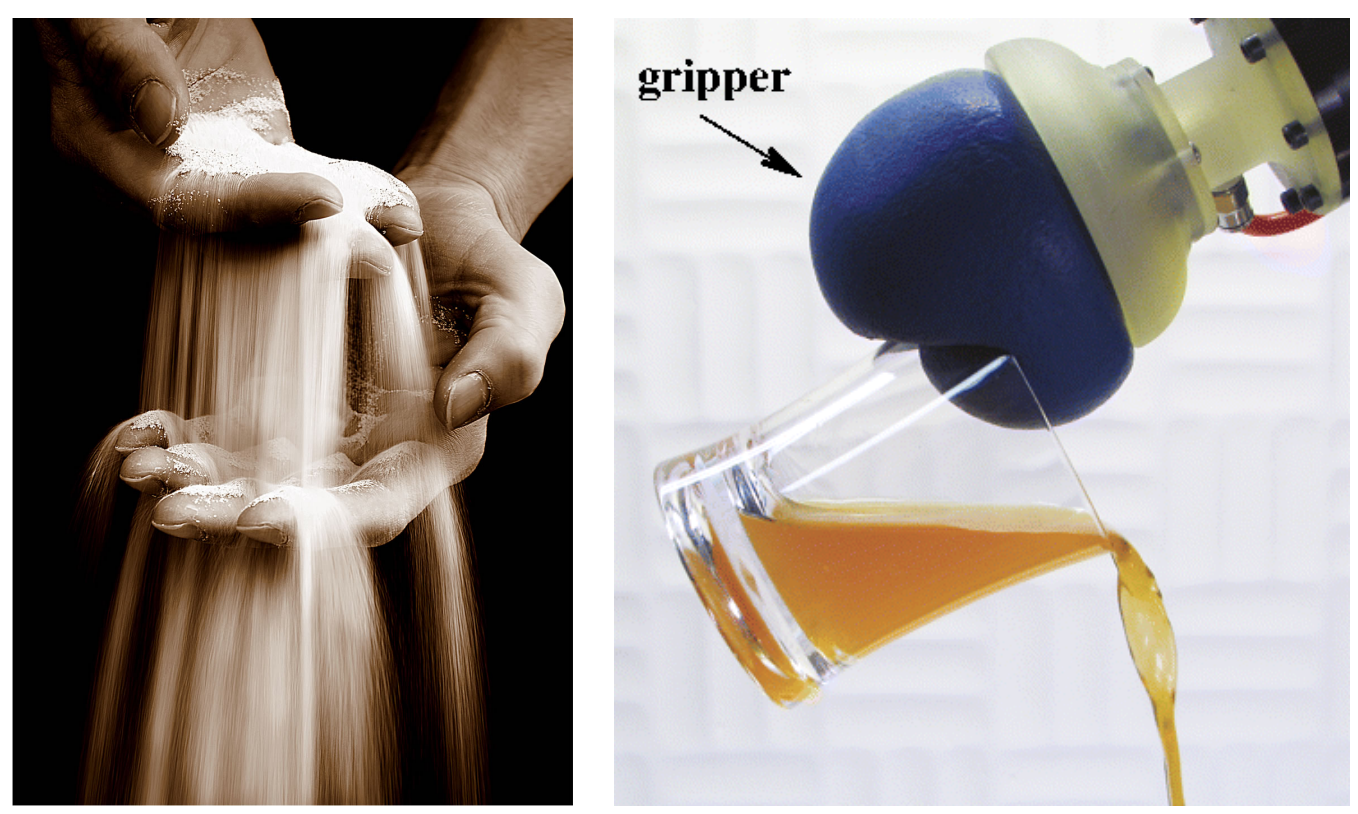

Fig. 1.1: Unjammed and jammed state of granular material. LEFT: Sands flowing through fingers. Picture taken by Thomas Kästner. RIGHT: The gripper consist of jammed ground coffee powder is holding the glass and pouring the water. The unjammed malleable gripper deforms around the edge of the glass, then harden when jamming is initiated by applying a vacuum [Brown et al., 2010.

Granular materials can resemble gases, liquids and solids, but they have essential differences from those materials. Granular materials are composed of particles larger than $100 \mu \mathrm{m}$. Thermal energy is negligible on this scale. Due to the dissipation, energy is not conserved during collisions such that a dense region will dissipate more energy, becoming even denser, resulting in the formation of a cluster of slow particles Goldhirsch and Zanetti, 1993, Mikkelsen et al., 2002, Weele et al., 2007]. In granular materials friction also plays an important role in both dynamic and static states. In some geological processes such as landslides, snow avalanches, granular flows in a non-Newtonian manner: 
the flow region is confined to a finite boundary layer and does not penetrate into the bulk of the material Komatsu et al., 2001. In static packings large force inhomogeneities are present [Liu et al., 1995]. Because of this, the propagation of sound in granular materials is qualitatively different from that in solids [Liu and Nagel, 1992]. This makes sands useful to provide vibration isolation or shock absorption. All above examples suggest that the transition between different states of granular materials can not be simply recognized as gas-liquid or liquid-solid transition. New physical descriptions are necessary for granular materials.

\subsection{Previous studies on length scales}

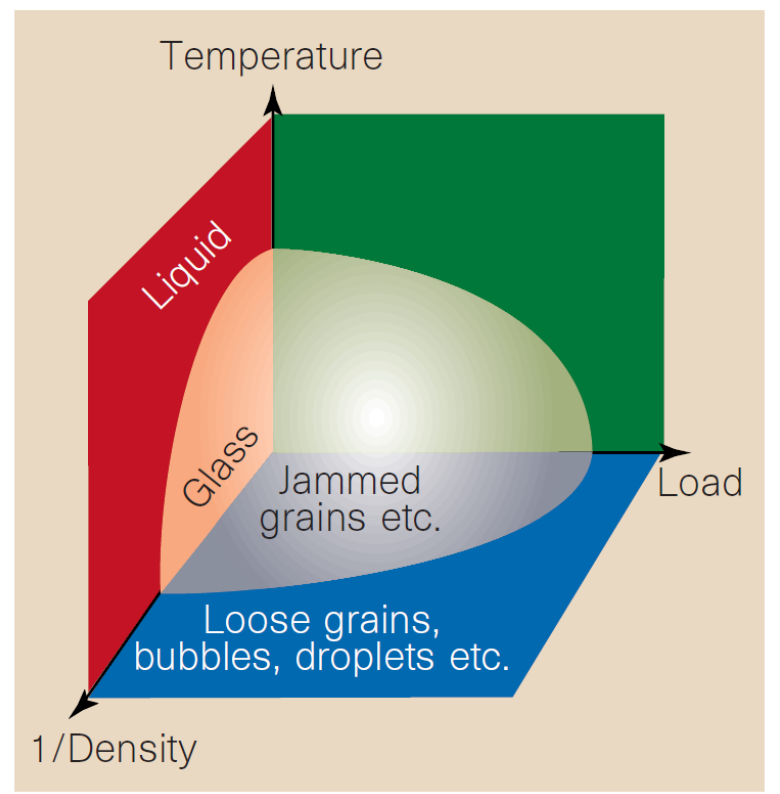

Fig. 1.2: A possible phase diagram for jamming Liu and Nagel, 1998. The jammed region, near the origin, is enclosed by the depicted surface. The line in the temperature-load plane is speculative.

It were Cates et al. [1998 who first proposed that granular material belongs to a new family of materials, the fragile matter. Similar to solids, the disordered system of this family can be driven into a jammed state by an external applied stress. Nevertheless, fundamentally different from ordinary solids, if the direction of the applied stress changes even by a small amount, the jammed state will break up. This may be due to the anisotropic force network [Majmudar and Behringer, 2005]. Liu and Nagel [1998] enlarged this family by including microscopic systems with attractive interaction such as glass, colloids. It is suggested that jamming transition is universal to those materials. This raises the question of whether there is a diverging length scale associated with the loss of rigidity. Although in a disordered sphere packing, no static order is expected to persist to long distance, the study on the structure factor revealed that the pair correlation has a power-law tail which is uncharacteristic of liquid states [Donev et al., 2005]. Such behaviour was found in the density fluctuations in the early Universe [Gabrielli et al., 2002] and in the ground 
state of liquid helium Reatto and Chester, 1967]. Studies of an idealized granular packing show that the jamming-unjamming transition has a mixed first-order-second-order character O'Hern et al., 2002, 2003]. As such a system unjams with decreasing packing fraction, the number of interacting neighbours per particle drops discontinuously to zero, while the power-law scaling is also observed for other quantities ['Hern et al., 2002. Silbert et al. 2005 extracted a corresponding length scale from the spatial variation of the vibration eigenmodes. They found this length scale diverging as a power law when approaching jamming from jammed side. Tewari et al. 2009 studied two-point temporal and spatial correlations of the coarse-grained velocity and stress fields in dense granular flow. The spatial correlations decay as a stretched exponential function. The corresponding length scales grow as a power law when approaching jamming.

According to the speculated jamming phase diagram, the tools used to study glass transition could be introduced in to granular system. There local dynamics are spatially heterogeneous. The spatial correlation length of the local dynamics grows as lowering the temperature. This topic will be discussed later in this chapter.

\subsection{The existence of correlation between local vol- umes}

Granular packing under certain disturbances, such as vibration and fluidization, can exhibits history independent and reversible states. By each disturbance the local configurations are changed, and the local volumes fluctuate around a steady value. One quantity characterizing the local volume is Voronoi volume $\mathrm{I}^{1}$. It has been found that spatial correlations exist between the fluctuation of Voronoi volumes by applying the central limit theorem.

Consider an array of random variable $v_{i}$ denoting the local volume which has some probability distribution $p_{i}$. This distribution has finite mean value $\bar{v}_{i}$ and standard deviation $\sigma_{i}$. The central limit theorem describes the relationship between the distribution of their sum $V_{N}$ and $p_{i}$. If the distributions of each variable in the array, $p_{i}$ are identical and independent of one another, then by the central limit theorem the distribution of $V_{N}$ would have mean value $N \bar{v}_{i}$ and its variance $N \sigma_{i}^{2}$ is extensive with $N$.

In a bidisperse system the dispersity breaks the first premise about the identity. Therefore the free volume, which is defined as the total volume occupied by each particle minus its hexagonal close packing volume, is used instead of the Voronoi volume ${ }^{2}$. In this context, the random variable $v_{i}$ is the free volume occupied by each grain, then $V_{N}$ is the total free volume of a cluster of $N$ grains. According to the central limit theorem, if the distribution of the free volume of individual grains satisfies the two premises above, the average free volume per grain in the cluster $v_{N}^{f}=V_{N} / N$ is constant with $N$ and the variance of that $\left(\sigma_{N}^{f}\right)^{2} \sim 1 / N$ is extensive. Otherwise at least one of the two premises stated above is violated. Lechenault et al. 2006] studied this idea. They computed the mean value and the standard deviation of the free volume of a cluster [cf. Fig. 1.3].

\footnotetext{
${ }^{1}$ Voronoi volume is the volume of a cell containing only one particle. Those cells tessellate the space. The definition of Voronoi cells and its generalization to bidisperse system is explained in Chapter 3 .

${ }^{2}$ The concept of free volume will be explored in detail in Chapter 4
} 


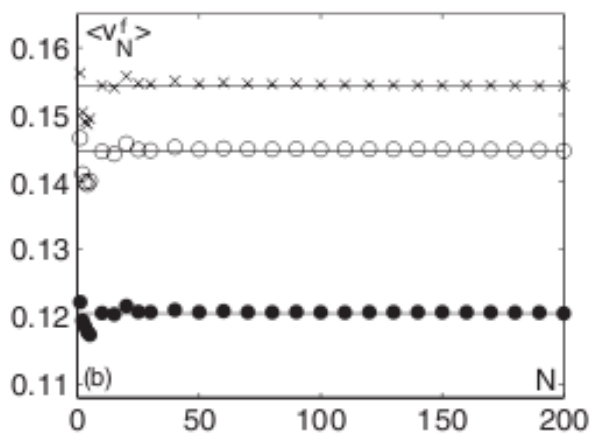

(a)

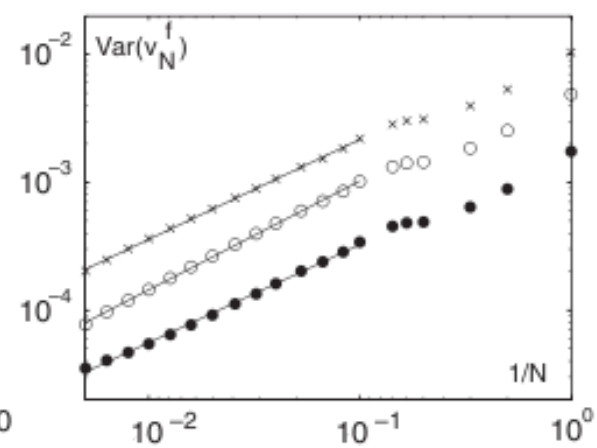

(b)

Fig. 1.3: Dependence on $N$ of mean value of the free volume per grain $\left\langle v_{N}^{f}\right\rangle$ (a) and standard deviation $\operatorname{Var}\left(\mathrm{v}_{\mathrm{N}}^{\mathrm{f}}\right)$ (b) for the three different experimental runs (non-vibrated $\operatorname{smooth}(\mathrm{o})$, and $\operatorname{rough}(\times)$ grains, and non-vibrated smooth grains $(\bullet))$. Curves in (b) are shifted for clarity [Lechenault et al., 2006].

In Fig. 1.3 (a) the mean of the free volume per particle $\left\langle v_{N}^{f}\right\rangle$ is constant with $N$, which indicates not only that the mean value of the free volume distribution of individual grain in the cluster is identical, but also the prediction about the mean value of the free volume of the cluster, $\left\langle V_{N}\right\rangle=N \bar{v}_{i}$. The result of the standard deviation or variance of the free volume of the cluster, however does not agree with central limit theorem. In Fig. 1.3(b) the exponent $\alpha$ in the scaling $\left(\sigma_{N}^{f}\right)^{2} \sim(1 / N)^{\alpha}$ is found to be 0.88 rather than 1 . Obviously the premise about the independence of the distribution of individual grain is violated. In other words, correlation exists. According to Fig. 1.3(b), the non-extensive scaling holds above $N=100$. The corresponding length scale would be larger than 6 diameter of particles.

\subsection{Statistical mechanics of granular packings}

Fig. 1.2 suggests that packing fraction, or the volume of granular systems is a crucial parameter to the jamming transition. Although the energy of granular systems is not conserved due to the intrinsic dissipation of the composing particles, the volume is conserved during the collisions between particles. Similar to the thermal system, granular materials are composed of large number of particles. This raises the interests to study the volume of granular materials in a statistical framework.

More than twenty years ago, Edwards and Oakeshott [1989] proposed a formulation closer to conventional statistical mechanics for static disordered granular assemblies. Following the spirit of statistical mechanics it describes a macroscopic system with a few parameters without having to deal with individual components. More explicitly, the volume plays the role of energy in conventional statistical mechanics, and the Hamiltonian should be replaced with a volume function $W$ that expresses the volume of the system as a function of the positions and orientations 3 of grains $\mathbf{q}$. The partition function is defined

\footnotetext{
${ }^{3}$ The particles are rigid.
} 
as:

$$
Z_{\chi}=\int e^{-W(\mathbf{q}) / \chi} \Theta(\mathbf{q}) \mathrm{d} \mathbf{q}
$$

Where $\Theta(\mathbf{q})$ imposes the mechanically stable condition which is essential to the jammed system; $\chi$ is the configurational temperature or compactivity more specified in granular systems. Equation 1.1 implies an assumption that for a given volume all mechanically stable configurations are equally probable. An 'effective volume' can be defined by $F=$ $-\chi \ln Z_{\chi}$, consequently the entropy $S=\partial F / \partial \chi$.

This suggestion has been studied intensively both in theory and in experiment. The history independent packing fraction sequence [Schröter et al., 2005] and the reversible states Brujić et al. 2005, Nowak et al., 1998] have been observed. These states are stable to perturbation, and have well-defined values of macroscopic quantities such as volume and global packing fraction. This kind of states might be analysed in an appropriate statistical framework. The volume function $W(\mathbf{q})$ has been built on Voronoi cells, and the distribution of the volume of cell was suggested to follow $\Gamma$ function Aste and Di Matteo, 2008, Aste et al. 2007]. Besides these purely geometrical considerations, a generalized ensemble includes the conservation of the force-moment tensor $\Sigma$ [Blumenfeld and Edwards, 2009]. In addition to compactivity $\chi$, there is an intensive variable that is conjugate to $\Sigma$, which is the angoricity tensor $\alpha^{-1}$. This ensemble is so termed as the Angoricity-compacitivity ensemble. Recently a generalization of Angoricity-compacitivity ensemble, the stress ensemble, has captured a lot of attention [Henkes and Chakraborty, 2009, Henkes et al., 2007, Lois et al., 2009]. On the basis of this ensemble Song et al. [2008] incorporated the mechanical stability condition and contact forces into a mesoscopic volume function, and used it to study the equation of state and the entropy Briscoe et al., 2008. Another microscopic building block of $W(\mathbf{q})$, quadrons, has been suggested as well Ball and Blumenfeld, 2002, Blumenfeld and Edwards, 2003]. Blumenfeld et al. [2012] showed that with quadrons the equipartition relation can be established between the volume and both structural and stress-related degrees of freedoms. Nevertheless this partition method may not be exact: the resulting $W(\mathbf{q})$ is smaller than the system volume when 'rattlers' exist [Ciamarra, 2007], which do not support other particles mechanically.

\subsection{Dynamic Heterogeneity}

An long-standing issue about glass transition is the origin of the slow dynamics observed as the critical temperature is approached. A possible picture is that molecules are rearranging in a cooperative manner. In the past two decades experiments have been developed to test this idea. It has been discovered that the dynamics of supercooled liquids and colloids is spatially heterogeneous - the particles in neighbouring areas have very different time scales of relaxation and particle motion. The size and span of those areas build up when approaching the glass transition [Ediger, 2000].

The slowdown of the dynamics in granular material was first reported by D'Anna and Gremaud 2001]. They examined the power spectrum of a torsion oscillator immersed in vibrated granular media. The inverse diffusivity diverges at a vibration intensity. This divergence is accounted for by an expression like the Vogel-Fulcher-Tammann form observed to describe glass transition [Debenedetti and Stillinger, 2001]. More microscopic 
observations were reported by Marty and Dauchot [2005], Pouliquen et al. [2003]. There the typical trajectories of grains display the cage effect and are similar to the ones observed in experiments on colloidal suspension and glass-formers.

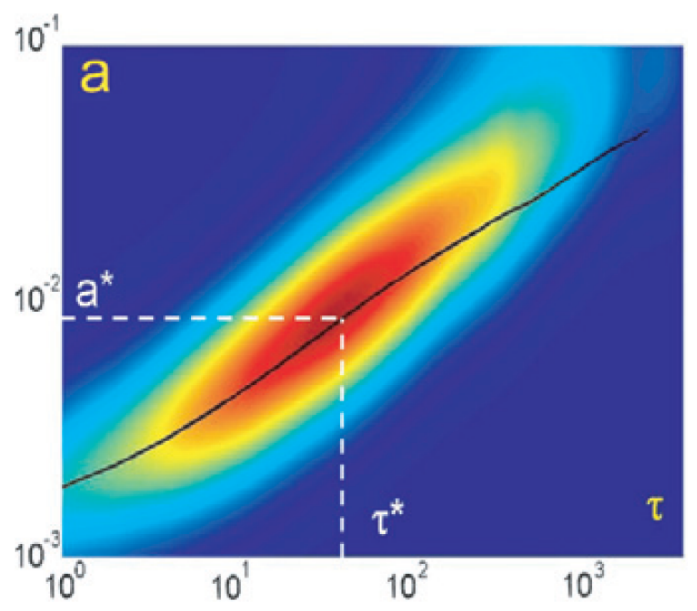

Fig. 1.4: $\chi_{4}(a, t)$ as a function of probing length $a$ and delay time $\tau$ for $\phi=0.8413$. The bold line represents the mean square displacement. Lechenault et al., 2008,

A direct quantitative measurement of dynamical correlations is the four-point correlation function $\chi_{4}$ [Latz, 2000]. Not so strictly speaking, $\chi_{4}$ can be understood as the fluctuation of decoupling time of density profile. This decoupling time itself is measured by density overlap function $Q(a, t)=\rho / N \int \mathrm{d} r q_{a}(r, t)$, where $q_{a}(r, t)=1 / \rho \int \mathrm{d} r^{\prime} \delta \rho(r, t) w_{a}(r-$ $\left.r^{\prime}\right) \delta \rho\left(r^{\prime}, 0\right)$ is the local temporal correlation. Where $\rho$ is the average density; $N$ is the number of particles. The overlapping function $w_{a}(r)$, which determines the locality of $q_{a}(r, t)$, can be chosen as a non-normalized Gaussian $w_{a}(r)=\exp \left(-r^{2} / 2 a^{2}\right)$ or a step function $w_{a}(r)=\Theta(r-2 a)$. Thus $\chi_{4}$ is defined as:

$$
\begin{aligned}
\chi_{4}(a, t) & =N\left\langle(Q(a, t)-\langle Q(a, t)\rangle)^{2}\right\rangle \\
& =\frac{\rho^{2}}{N} \int \mathrm{d} r \mathrm{~d} r^{\prime}\left\langle\left[q_{a}(r, t)-\left\langle q_{a}(r, t)\right\rangle\right]\left[q_{a}\left(r^{\prime}, t\right)-\left\langle q_{a}\left(r^{\prime}, t\right)\right\rangle\right]\right\rangle \\
& =\rho \int G_{4}^{a}(r, t) \mathrm{d} r
\end{aligned}
$$

Where $G_{4}^{a}(r, t)=\left\langle\left[q_{a}(r, t)-\left\langle q_{a}(r, t)\right\rangle\right]\left[q_{a}(0, t)-\left\langle q_{a}(0, t)\right\rangle\right]\right\rangle$ is the spatial correlation of local temporal correlation. Therefore Eq. 1.2 states that large value of $\chi_{4}(a, t)$ has to be related to long range spatial correlations of local temporal correlation, a measurement of the dynamical correlation length both in time and in space. An example of $\chi_{4}(a, t)$ measurement can be seen in Fig. 1.4. The first DH measurement in granular media was performed by Dauchot et al. [2005] at a fixed packing fraction. Keys et al. [2007] and Lechenault et al. 2008] presented the evolution of dynamical heterogeneities as a function of the packing fraction near jamming transition. All these experiments have either been performed in two dimensional systems [Dauchot et al., 2005, Keys et al., 2007] or at the boundaries of three dimensional systems. 


\subsection{Outline of the thesis}

This thesis consists of two parts: 2D disc packings (Chapter 2 to Chapter 5) and 3D sphere packings (Chapter 6 to Chapter 8). In Chapter 4 the idea of the scaling the variance of the volume of a cluster with the cluster size is tested, then extended to measure the correlation between the fluctuation of local volumes in bidsperse disc packings. Anticorrelation is observed above an onset packing fraction. Three lengths are extracted from the correlation, and their evolution with $\phi$ is studied. In Chapter 5 we measure $\chi$ on Voronoi cells in disc packings by three different methods and show that the uniform $\chi$ can only be defined above a cluster size characterized by the correlation length between local volumes.

In Chapter 8 the correlation measurement and the compactivity measurement are performed on sphere packings. We also measured the dynamics of sphere packings in a fluidized bed by fast X-ray tomography and observed some evidence about the dynamic heterogeneity. The limitation of the experiment will be explained together with the experimental protocol in Chapter 6 . 


\section{Part I}

\section{Packings in 2D}




\section{Chapter 2}

\section{Experimental Protocol}

Experiments are performed in a two-dimensional air fluidized bed, of which a photo is shown in Fig. 2.1. The bed consists of a mixture of two sizes of circular discs confined between glass plates separated by a distance slightly larger than the thickness of the discs. The packing is tapped from below by air pulses the duration and strength of which can be varied. Following each tap a image is taken by a camera positioned in front of the bed. A schematic is shown in Fig. 2.2. In this chapter we will explain each part of the experimental protocol in detail.
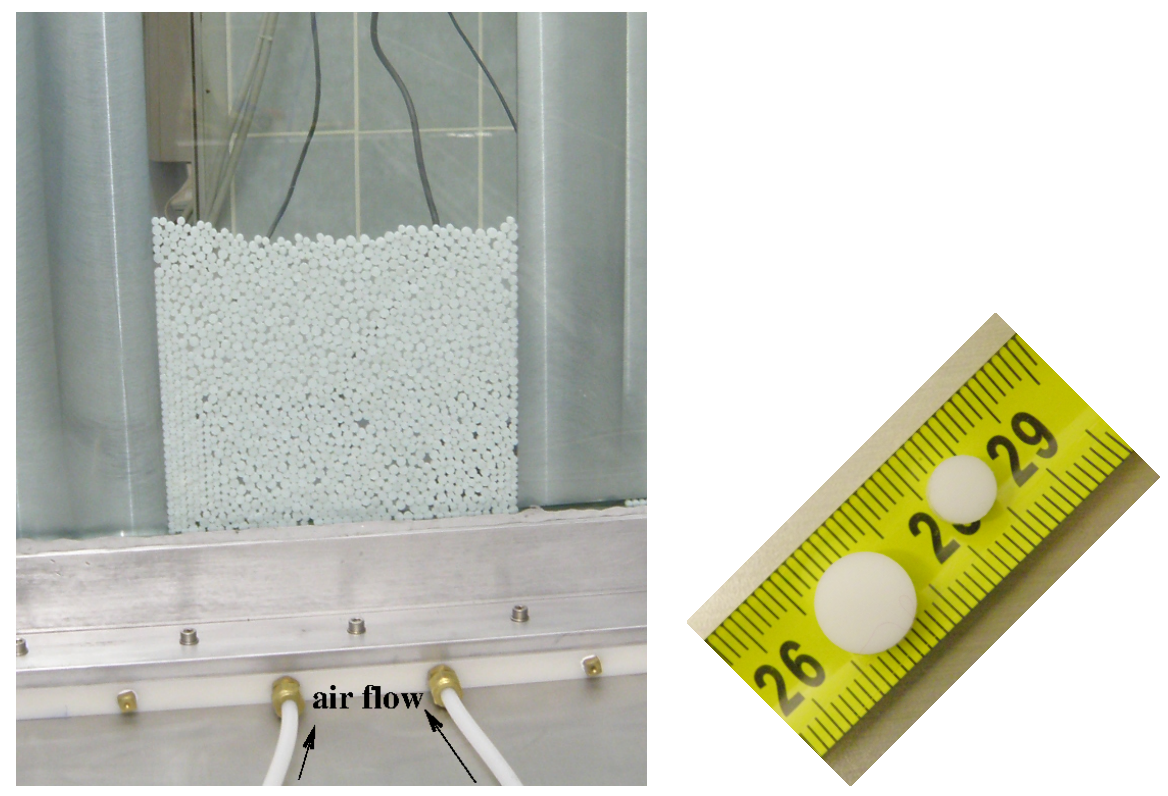

Fig. 2.1: The 2D fluidized bed and the dimension of the discs. The bed confined between two glass plates is put vertically. Air is sent through a distributor to fluidize the bed. The bed consists of a binary mixture of discs. The diameter of large and small discs are $9 \mathrm{~mm}$ and $6 \mathrm{~mm}$.

\subsection{Two-dimensional bed}

The particles are a binary mixture of Teflon discs with diameters of $d_{s}=6 \mathrm{~mm}$ and $d_{l}=9 \mathrm{~mm}$ and $4.15 \mathrm{~mm}$ thick ( Fig. 2.1). Approximately 750 discs of each size comprise the bed. The Teflon discs have very smooth edges resulting in a better quality image and a more homogeneous system.

The bed consists of two pieces of float glasses, each $1.2 \mathrm{~cm}$ thick and measuring 53 $\mathrm{cm}$ wide by $48 \mathrm{~cm}$ high. The glass is mounted vertically with spacers between the plates creating a gap 1.1 times the thickness of the particles (about $4.56 \mathrm{~mm}$ ), ensuring that the particles are able to move freely without tilting in the two-dimensional plane. The edges of the spacers are glued with a layer of Teflon with the same friction coefficient as the discs used in the experiment. 

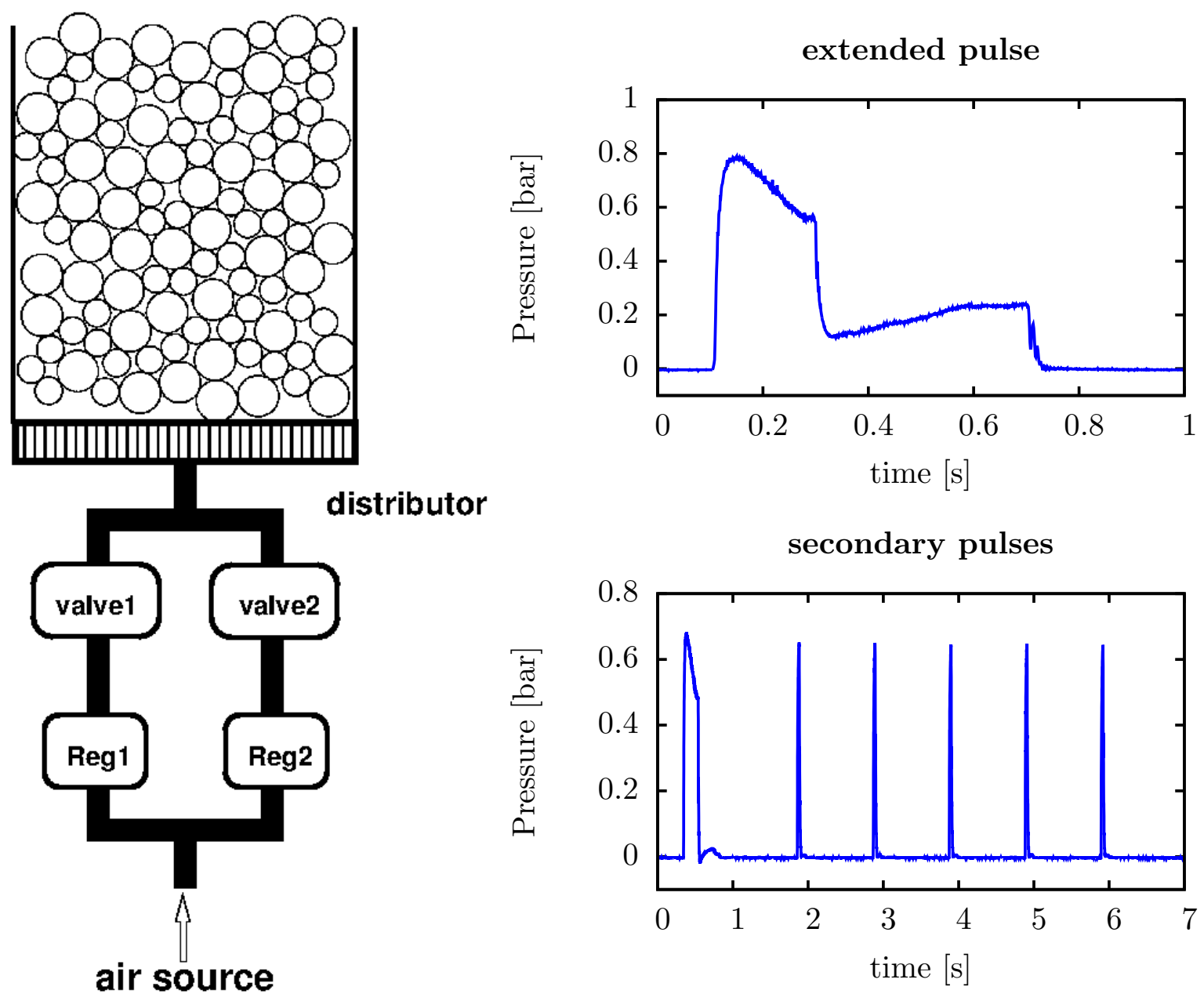

Fig. 2.2: LEFT: the sketch of the $2 \mathrm{D}$ setup. RIGHT: examples of two tapping modes. The pressure is measured by the sensor below the distributor.

Three sensors are installed in the base of the bed, below the distributor to monitor various quantities during the course of the experiment. The pressure sensor is a variable pressure transducer (Validyne DP15-22) with diaphragm number 42, for which the full scale pressure is 20 psi. Relative humidity is measured by a humidity sensor (Honeywell HIH-3610-002), and the temperature is measured by a precision thermistor (YSI 44033). The data from the sensors is transferred to a 16-bit data acquisition card (National Instruments PCI-6036E) and saved. Typical humidity and temperature are $3.8 \pm 0.3 \%$ and $24.8 \pm 0.4^{\circ} \mathrm{C}$.

\subsection{Tapping}

Air is sent through a distributor of open-porous foam (Duocel 40 PPI aluminium foam) to fluidize the bed. In early experiments the signal of the pressure sensor has spikes after several tappings and the spikes disappeared by manually disturbing the bed with a metal rode. It turns out that this is due to the electrostatic charging on the distribution. Grounding the distributor minimize the electrostatic charging and recover the 
Tab. 2.1: Parameters of the air pulses. All the experiments start with the same primary pulse (3.2bar measured at the regulator, $200 \mathrm{~ms}$ ), either followed by several secondary pulses with the same pressure or accompanied by an extended pulse.

\begin{tabular}{|ccc|}
\hline$\phi_{\text {avg }}$ & Extended pulse & Secondary pulses \\
\hline 0.8175 & 0.5 bar $\times 1000 \mathrm{~ms}$ & - \\
0.8183 & 0.45 bar $\times 1000 \mathrm{~ms}$ & - \\
0.8209 & - & 0 \\
0.8218 & - & $5 \times 15 \mathrm{~ms}$ \\
0.8231 & - & $10 \times 15 \mathrm{~ms}$ \\
0.8256 & - & $3 \times 30 \mathrm{~ms}$ \\
0.8277 & - & $7 \times 30 \mathrm{~ms}$ \\
0.8315 & - & $15 \times 30 \mathrm{~ms}$ \\
0.8337 & - & $20 \times 30 \mathrm{~ms}$ \\
0.8356 & - & $15 \times 30 \mathrm{~ms}+15 \times 15 \mathrm{~ms}$ \\
0.8366 & - & $30 \times 30 \mathrm{~ms}+30 \times 15 \mathrm{~ms}$ \\
0.8380 & - & \\
\hline
\end{tabular}

measurement of the sensors.

The duration and strength of air pulses are controlled by two Waston Smith 06B04604 mechanical pressure regulators (Reg1 and Reg2 in the sketch in Fig. 2.2) and two pairs of Jefferson 2026 series electronic valves (valve1 and valve2 in the sketch in Fig. 2.2). The airflow through the two airflow pathways, Reg1 \& valve1 and Reg2 \& valve2, can be controlled independently. The average packing fraction $\phi_{\text {avg }}$ value is controlled by the type and duration of the air pulses.

The two pathways are used to design two tapping modes. The most compact beds are obtained by first driving the bed to a new configuration using a primary pulse, and then following that by shorter secondary pulses to compactify the bed [cf. Fig. 2.2]. The duration of the primary pulse and the strength of air flow are fixed, but the number and duration of the following secondary pulses are adjustable. Looser packings are obtained using two airflow pathways together but with different flow rates. The two pathways are switched on at the same time, then the primary pulse stops first. When the primary pulse stops, the secondary pathway still provides some small flow, which slows down the settling discs [cf. Fig. 2.2].

The use of different tapping modes enables us to vary $\phi_{\text {avg }}$ from 0.8175 to 0.8380 . Parameters are listed as shown in Tab. 2.1.

\subsection{Illumination and imaging}

During the experiment the bed is illuminated from behind by a $28 \times 56$ array of red LEDs diffused through a translucent piece of plastic. The LED array is powered by a DC power supply (VOLTCRAFT VSP 2405HE) set at $24 \mathrm{~V}$ and 5 A.

Images are taken by a Sony XCD-100 digital camera (1.9 megapixel) with a Nikkor 
$50 \mathrm{~mm}$ lens. Only the central region of the packing $\left(252 \times 192 \mathrm{~mm}^{2}\right)$, five small disc diameters away from boundaries, is captured. This setup allows an approximate resolution of 59 pixels/cm. The corresponding diameter of small and large disks are 35.4 pixel and 53.1 pixel.

\subsection{Experimental procedure}

Before each experiment the bed is first put into a random mixed state by stirring the particles manually. A certain tapping protocol is selected and a series of 8000 cycles of taps are done. Following each cycle of taps, the bed is allowed to relax for 4 seconds and an image is taken. The processing of those images are explained in Chapter 3 . 


\section{Chapter 3}

\section{Image Processing}

The positions of particles in a granular packing are essential to 'microstate' analysis. This chapter first explains how to process the image of a bidisperse disc packing to get the centroids and disc types, then exhibits two methods of local volume tessellation for bidisperse systems.

\subsection{Determining particle centroids}

The identification of the centroids of discs in a raw image consists of several steps: binarization, erosion, identification of types and centroids and correlation correction. All the processes are done using the Image Processing toolbox in the Matlab development. The steps are explained below, with the results of each step shown in Fig. 3.1.

Step One: Binarization. Ten images are taken of the bed without particles under the same illumination conditions as during an experiment. These ten images are averaged to form the background image. This is then divided out of the raw image to remove any inhomogeneities resulting from the illumination [cf. Fig. 3.1(a),(b),(c)]. The resulting gray scale image is converted to a binary image with the threshold determined by Otsu's method. The pixels with a gray value greater than this threshold are changed to 0 (black) and the others to 1 (white), resulting in an image of only black and white pixels [cf. Fig. $3.1(\mathrm{~d})]$.

Step Two: Erosion. Following this binarization, a series of erosions are performed on the image. The rule of erosion is as follows: the output pixel value will be the minimum value of all pixels in a predefined neighbourhood. In this case the neighbourhood is chosen to be a disk with three-pixel-radius. In the case of a binary image, if any pixel in the neighbourhood of the input pixel is black (0), then black (0) is set to be the output of that pixel. The erosion is applied to every pixel in the inverted binary image, resulting in a shrinking of the circular particles. Several erosions are performed to completely separate the particles from each other.

Step Three: centroids and types. After the erosions, the image consists of many separated black regions in a white background [cf. Fig. 3.1(e)]. Every black region is recognized as a particle. The centroid and area of each black region can be calculated. The type, small or large, is determined according to the area. From the above three steps we get a list of $x$ and $y$ coordinates as well as the type for every particle in the image. These data is enough to characterize a packing. See Fig. 3.1(f) for packing reconstruct from the processed data and the original image.

Step Four: correlation correction. The resulting centroids computed from the erosion process may contain artefacts. One potential artefact is that the difference of the intensity near contacting point and at a free boundary affects the binarization and the erosions. For instance the 'neck' between contacting particles can be seen in the binary image in Fig. 3.1(d). This makes the result of the erosions for a particle dependant on the number and spatial configuration of the particle's touching neighbours. It is 

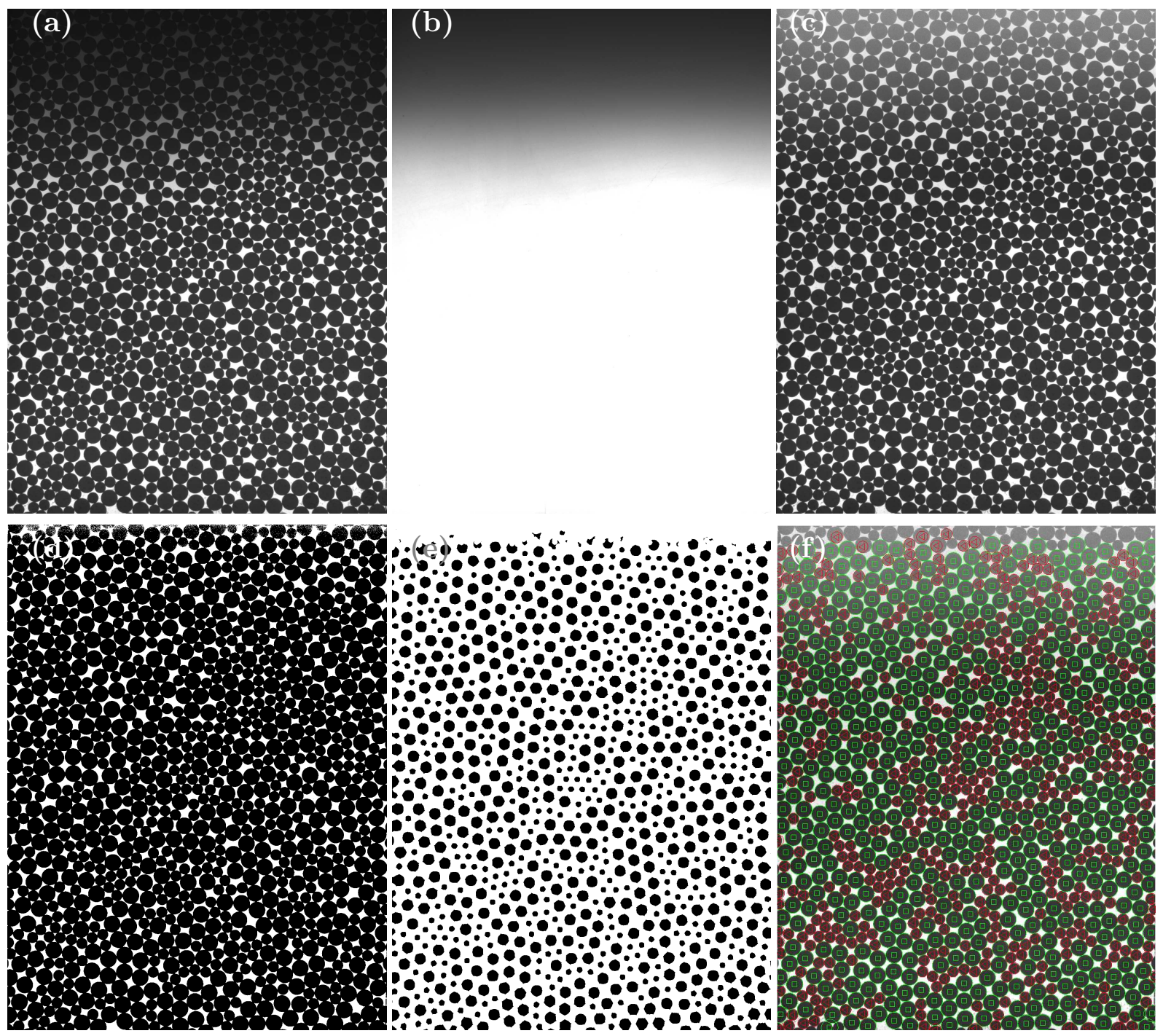

Fig. 3.1: (a): A raw image taken during the experiments. (b): Illumination image without discs which is used as the background for intensity correction. (c): The raw image divided by the background image. (d): The binary image of (c). (e): Apply erosion twice to the binary image. (f): Centroids and types identified from the image after the erosions are labelled by symbols and colors. Large discs are green circle and small ones are red.

therefore necessary to perform a cross-correlation on the original image with a template, which is a perfect filled circle with known size and centroid, and adjust the centroid coordinates accordingly. The cross-correlation is done using a slightly modified version of the Matlab function cpcorr. For each particle found by the erosion process, cpcorr finds the maximum of the convolution map then fits a parabola around the maximum within a $10 \times 10$ pixels region [cf. Fig. 3.2 (c)]. The coordinates of the maximum of the parabola fit is extracted. The centroid coordinates of the particles are adjusted according to the coordinates of the maximum of the fit. 


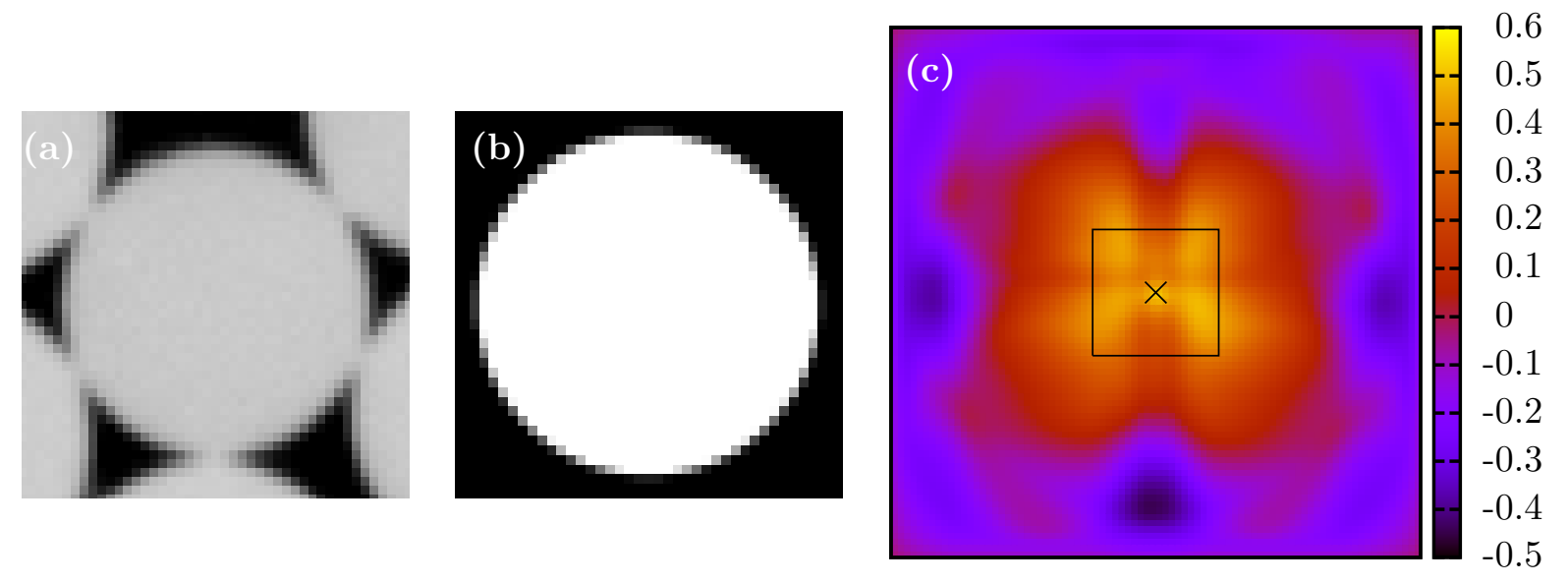

Fig. 3.2: (a): a disc in a raw image. (b): a template disc. (c): the convolution map of the template on the raw image. The cross denotes the maximum of the convolution map. The square denotes the $20 \times 20$ pixels region which is used to do the parabola fit.

\subsection{Local volume tessellation}

To study the properties of local volumes in a packings, one need first tessellate the space into local cells, then the local volumes are defined as the volume of the local cells. Voronoi tessellation is a popular tool and has been used to analyse both static and dynamic behaviours of granular systems Aste and Di Matteo, 2007, 2008, Aste et al., 2007, Briscoe et al., 2008, Kumar and Kumaran, 2005, Lechenault et al., 2006, McNamara et al., 2009, Puckett and Daniels, 2012, Puckett et al., 2011, Slotterback et al., 2008, Song et al., 2008].

Imaging a system of a finite set of sites, $p_{n}$, on a plane. The Voronoi cell corresponding to $p_{n}$ consists of every point whose distance to $p_{n}$ is less than or equal to its distance to any other site. Each such cell is obtained from the intersection of half-planes, and hence it is a convex polygon. The segments of the Voronoi diagram are all the points in the plane that are equidistant to the two nearest sites. This definition can be easily generalized into mono-size particle system using the site $p_{n}$ denote the center of particles. However for the bidisperse systems the definition must be adapted. In this section two modified tessellation methods, navigation map and radical tessellation, are introduced. Both methods would be reduced to the normal Voronoi tessellation when the bidisperisty vanishes.

\subsubsection{Navigation map}

One way to tessellate a bi-disperse disc packing is the navigation map. This method takes a Voronoi cell as the collection of points closer to the surface of that occupying particle rather than to the centroid. According to this definition, any points on the edge of a Voronoi cell must satisfy the following equation: 


\section{navigation map}

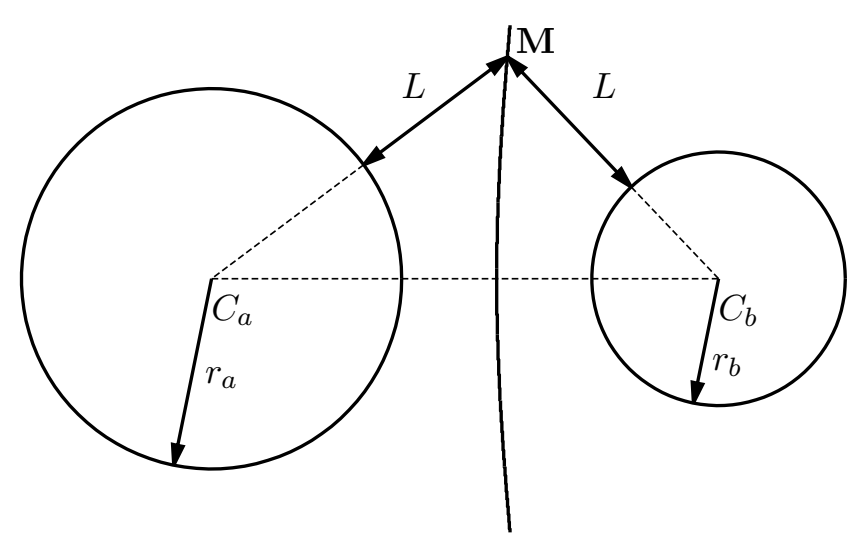

Fig. 3.3: The definition of navigation map. $C_{a}, C_{b}$ are the centroids and $r_{a}, r_{b}$ are the radius of particle $a, b$ respectively. The solid curve between particle $a$ and $b$ is the edge of Voronoi cell separating them. $\mathrm{M}$ is a point on the edge. The double headed arrows indicate the distance from $\mathrm{M}$ to the surfaces of particle $a$ or $b$. According to the definition of Voronoi cell in Eq. 3.1 these two distances should equal $L$.

$$
\sqrt{\left(x-x_{a}\right)^{2}+\left(y-y_{a}\right)^{2}}-r_{a}=\sqrt{\left(x-x_{b}\right)^{2}+\left(y-y_{b}\right)^{2}}-r_{b}
$$

$x, y$ are the coordinates of the point on the edge, $x_{a}, y_{a}, x_{b}, y_{b}$ are the coordinates of the centroids and $r_{a}, r_{b}$ are the radii of two neighbouring particles, $a$ and $b$, respectively [cf. Fig. 3.3.

The solution of Eq. 3.1 is hyperbolic. Because of this, analytic calculation of the vertices and faces of the cells, as well as the areas becomes very difficult. Instead, a numerical determination of the cells was implemented.

The straightforward method for computing the navigation map is to calculate the distance from every pixel to every particle surface in the system.

$$
d_{i}=\sqrt{\left(x-x_{i}\right)^{2}+\left(y-y_{i}\right)^{2}}-r_{i}
$$

$d_{i}$ is the distance from the pixel $(x, y)$ to the surface of the particle at $\left(x_{i}, y_{i}\right)$ with radius $r_{i}$. The pixel $(x, y)$ belongs to the particle $i$ which gives the shortest $d_{i}$. The computation is very time consuming and only gives the cell volume in pixel resolution. Two methods are applied to save the computation time while getting a sub-pixel resolution map.

For a given particle any pixels of the particle must be assigned to the particle. In practise a circle with a radius 1.2 times larger than that of the particles is used instead. The 'grown' particles help assign inter-particle pixels but the neighbouring particles overlap resulting in conflicting assignments. When this happens, the particles to consider are reduced to the two overlapping neighbouring particles, and Eq. 3.2 has to be applied only to these two particles. Now, only pixels in inter-particle space have to be assigned according to the distances, $d_{i}$, to the particles in their immediate vicinity. Fig. 3.4(a) shows a demonstration at a one pixel resolution.

The one-pixel navigation map has all pixels assigned. Next, we want to get higher resolution result. There are two kinds of pixels in the one-pixel navigation map. One kind 

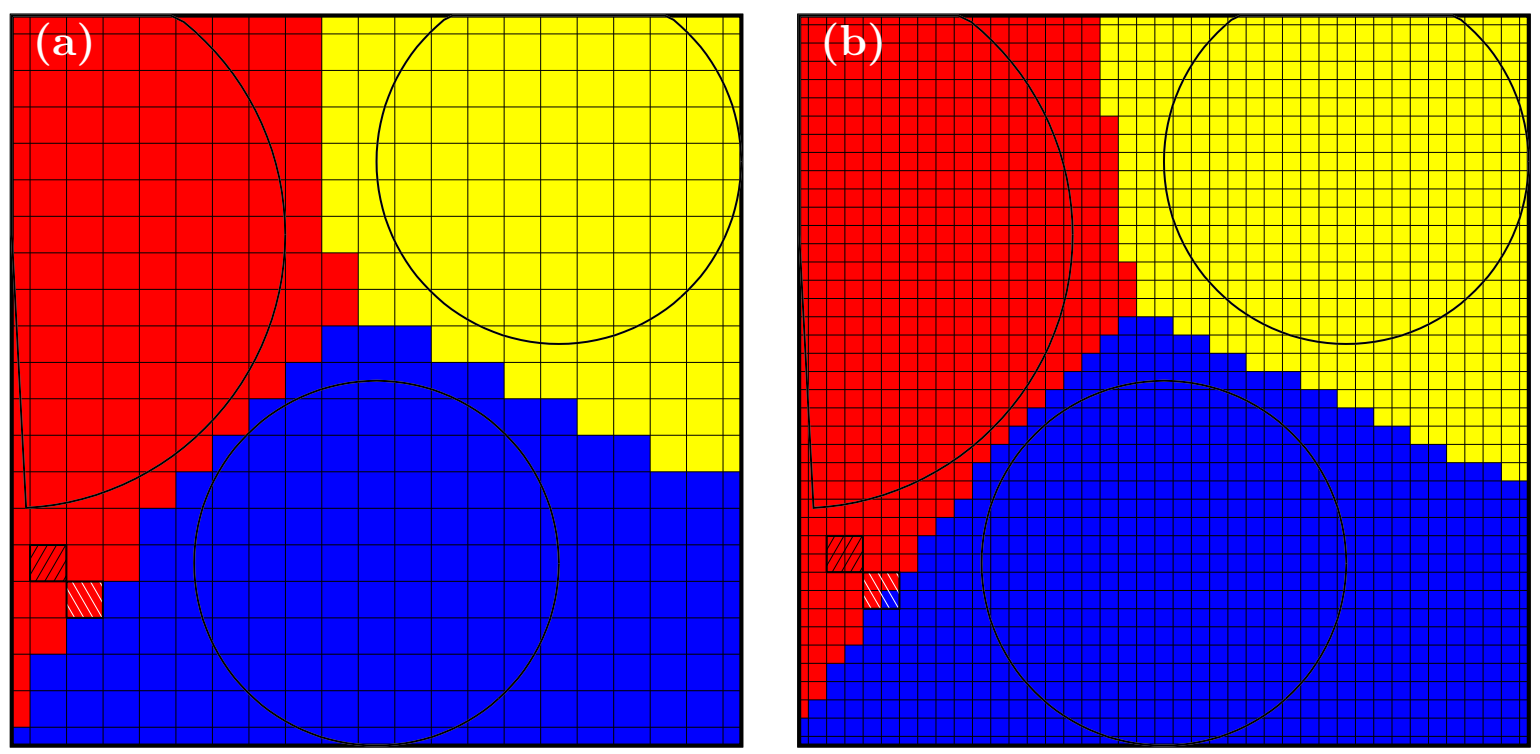

Fig. 3.4: Navigation map computed with (a) pixel resolution and (b) $\frac{1}{4}$-pixel resolution (one pixel divided into four sub-pixels). The discs are not plotted in scale. In (a) the pixel filled with black pattern has all its neighbours belonging to the same cell (red), so the corresponding sub-pixels in (b) are assigned directly to the red cell. The pixel filled with white pattern has two neighbours belonging to the blue cell, so in (b) one of its sub-pixel is assigned to the blue cell.

are the pixels in a given cell which do not have neighbouring pixels of another cell. The other kind is the pixels on the edges of the cells which have at least one neighbour assigned to other cells. When computing a higher resolution navigation map, the sub-pixel of the first kind are assigned directly to the cell of its parent pixel. The sub-pixel of the second kind is assigned by according to the shortest distance computed in Eq. 3.2. A demo is plotted in Fig. 3.4(b). In practise every pixel is divided into 16 sub-pixel, i.e. $\frac{1}{16}$-pixel resolution.

\subsubsection{Radical tessellation}

The other tessellation method considered was radical tessellation. Radical tessellation takes the edge of the cell as the collection of points whose tangents are equal relative to neighbouring particles [cf. Fig. 3.5]. The cells computed by this method are referred to as the radical cells. The calculation of the radical cells involves merely solving the following equation:

$$
\left(x-x_{a}\right)^{2}+\left(y-y_{a}\right)^{2}-r_{a}^{2}=\left(x-x_{b}\right)^{2}+\left(y-y_{b}\right)^{2}-r_{b}^{2},(a \neq b)
$$

$x, y$ are the coordinates of the point on the edge, $x_{a}, y_{a}, x_{b}, y_{b}$ are the coordinates of the centroids and $r_{a}, r_{b}$ are the radii of two neighbouring particles, $a$ and $b$, respectively [cf. Fig. 3.5. In a mono-size packings, $r_{a}=r_{b}$ can be eliminated in Eq. 3.3, so that radical tessellation goes back to the normal Voronoi tessellation where the points $(x, y)$ on the edge are equidistant to the centroids of the two particles. 
radical tessellation

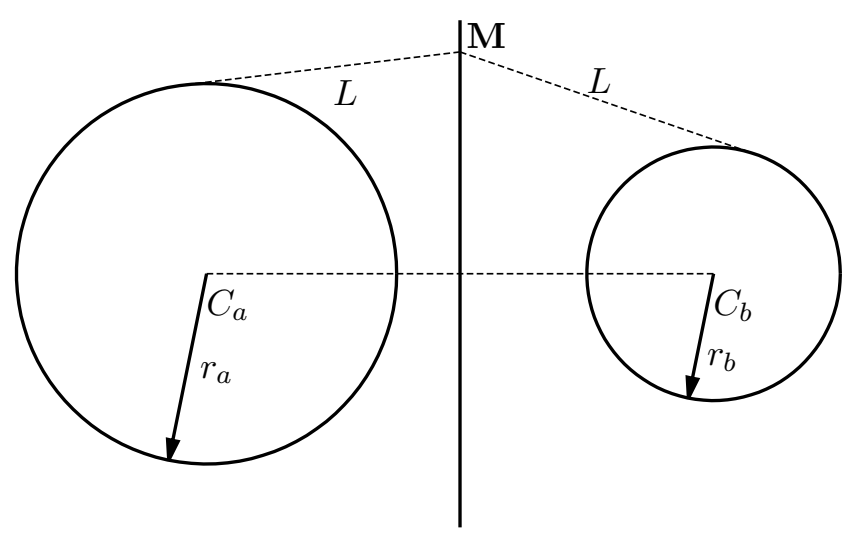

Fig. 3.5: The definition of radical tessellation. $C_{a}, C_{b}$ are the centers and $r_{a}, r_{b}$ are the radius of particle $a, b$ respectively. The solid line between particle $a$ and $b$ is the edge of Voronoi cell separating them. $\mathrm{M}$ is a point on the edge. The dash lines indicate the tagents from $\mathrm{M}$ to the particle $a$ and $b$. According to the definition of Voronoi cell in Eq. 3.3 these two distances should equal $L$, and the edge of the Voronoi cell is a straight line and perpendicular to the line connecting $C_{a}$ and $C_{b}$.

The solution of Eq. 3.3 is a straight line perpendicular to the line connecting the two centroids, $C_{a}$ and $C_{b}$. [cf. Fig. 3.5. This line is the shared edge of radical cells corresponding to the two particles. With another particle which is a neighbour to either $a$ or $b$ or both, another cell edge can be obtained, and the intersection of the edges must be a vertex of the cell, which has the equidistant tangents to all three particles. In other words, the three neighbouring particles forming a triangle which gives a vertex shared by all the three particles. A radical cell can be determined as follows: first find all neighbours of a particle, then group the particle and its neighbours into triangles, and each triangle gives a vertex of the cell of the particle, finally connect all vertex to get the cell.

In normal Voronoi tessellation the neighbours of a particle and the triangles of neighbours are calculated by the Delaunay triangulation. In a set of points one can construct a circumscribed circle for any three points. If the circumscribed circle contains no other points, the center of the circumscribed circle are equidistant to all three points, and no other points are closer to this center than the three points. Therefore the center of the circumscribed circle must be a vertex shared by the three points. However for the bidisperse system Eq. 3.3 is not consistent with this triangulation algorithm. Using the Delaunay triangulation and Eq. 3.3 together may generate wrong tessellations which results in the overlapping radical cells as shown in Fig. 3.6. The triangulations should be checked.

In the correct triangulation case, the cells of the three particles have at least two edges shared with one another. Any incorrect triangulation results in the overlapping of neighbouring radical cells, which reduces the number of shared edges to one. This can be concluded as that the triangulation is wrong if its composing particles only has one shared edge.

This gives the criteria to locate the incorrect triangulation. Additionally, any incorrect triangulation indicates that one of its neighbouring triangulation is also incorrect. Two 


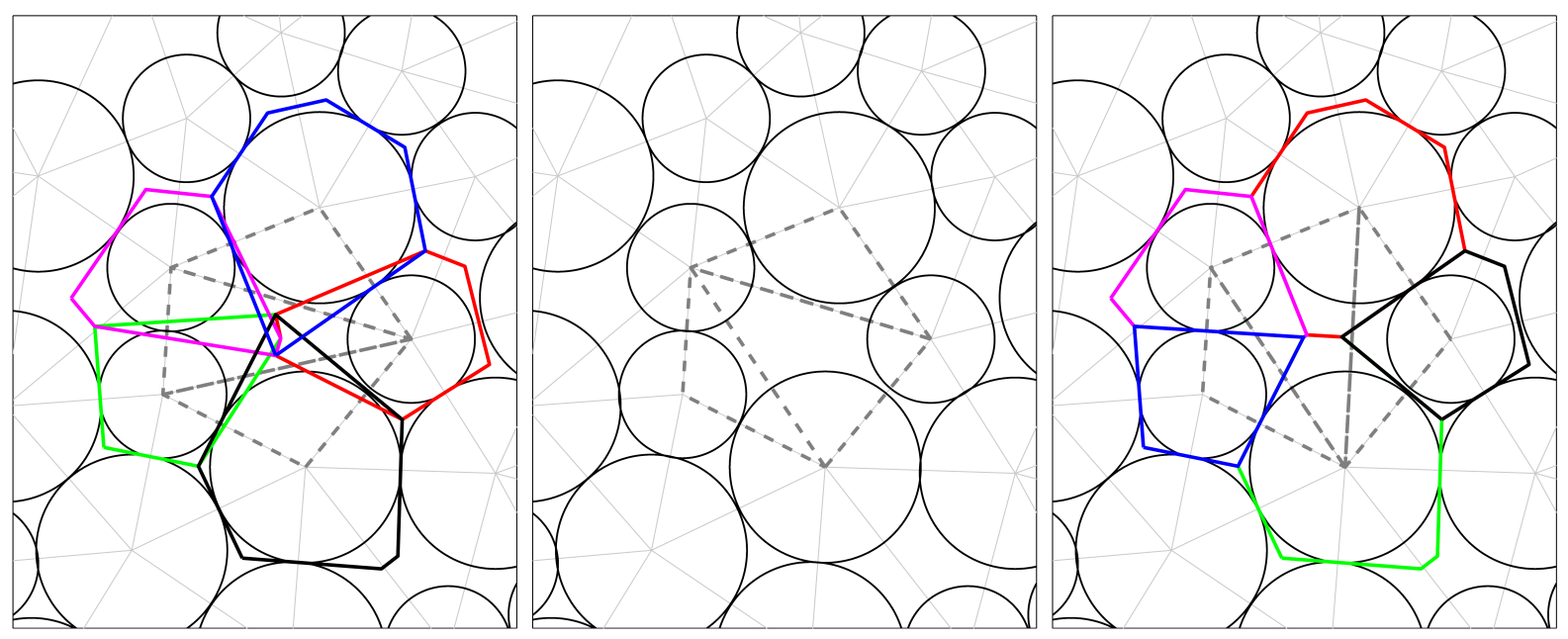

Fig. 3.6: LEFT: uncorrected tessellation. The three neighbouring dashed triangles are the wrong triangulations. The cells (coloured polygons) are computed by Eq. 3.3. The three vertex of cells are given by such triangulations resulting in the overlapping of the cells. In total two shared edges are found between cells: (magenta \& red) and (green \& red). MIDDLE: First the bottom and middle triangle in left panel are swapped. RIGHT: The middle and the top triangles in the middle panel are swapped. New vertices are computed with the correct triangulations. All neighbouring cells share one edge.

neighbouring triangles form a quadrilateral. One has to change the triangulation so that the quadrilateral is divided along the other diagonal to get the correct triangulation. This process is called swapping. Sometimes the above criteria is not sufficient to identify which neighbouring triangle should be swapped with. For instance, in the case shown in Fig. 3.6, three neighbouring Delaunay triangles (gray dashed line), five cells (color solid line) are involved. From bottom to top the three triangles are labelled as 1, 2, 3. Triangle 1 has only one shared edges among its cells, so does triangle 3 . Thus triangle 1 and 3 are incorrect. However, their common neighbour, triangle 2 does have two shared edges (red and green cell, red and magenta cell), although apparently the tessellation is wrong. To locate triangle 2 as the incorrect triangulation as well, one needs to check whether two particles in the triangle share a vertex but have no shared edge (green and magenta cell). Then to correct such an error, one swaps the three triangulations in sequence: first swap triangle 1 and 2 to get new triangles 1' and 2' (middle panel in Fig. 3.6), then swap 2' and 3 to get the correct triangulation (right panel in Fig. 3.6).

In practise the radical tessellation is fulfilled in such a procedure: the Delaunay triangulation is performed; for each triangle the vertex is computed according to Eq. 3.3 ; the number of shared edges is checked for every single triangle, and the triangles with only one shared edge is labelled into the set $w_{-}$tri; if one of its neighbouring triangle is also in $w_{-}$tri, they are swapped, new vertexes are calculated; if a triangle in $w_{-}$tri has no neighbour in the set, all its neighbours are checked and corrected as in Fig. 3.6; finally go through all triangulations again to make sure no more error left. In the dense packings the incorrect triangulations are rare. The instance in Fig. 3.6 may happen at arches or bridges, where a void is surrounded more than four particles.

Radical tessellation method can be solved analytically, so that it has been generalized 
to polydisperse in three-dimension packings, for instance, the popular open source package voro++. Nonetheless we found that the radical tessellation does not fit our purpose of analysis well. Henceforth without specifying the analysis is done on the local volume computed in navigation map, and Voronoi volume is used interchangeable with this local volume. These two tessellation methods will be compared in Chapter 4 . 


\section{Chapter 4}

\section{Correlation between Voronoi volumes in disc packings}

As already explained in Chapter 1, the non-extensive scaling of the volume fluctuation of a cluster composed of Voronoi cells implies the existence of a correlation between Voronoi volumes [Aste and Di Matteo, 2007, Lechenault et al., 2006. This correlation, however, had never been measured and studied directly. In this chapter we will first verify the validate of the experimental data and the concept of free volume, then use the nonextensive scaling argument to define a proper measure of the correlation between Voronoi volumes, finally three lengths are extracted to characterize the evolution of the correlation with the average packing fraction.

\subsection{Ensuring a steady and homogeneous state}

In order to be able include every tap in the overall set of data, it is necessary to ensure that during the course of the experiment the system was in a steady state. The steady state in this context means that even though the configuration of individual grains was changed after each cycle of tapping, nothing was qualitatively different about the macroscopic state.

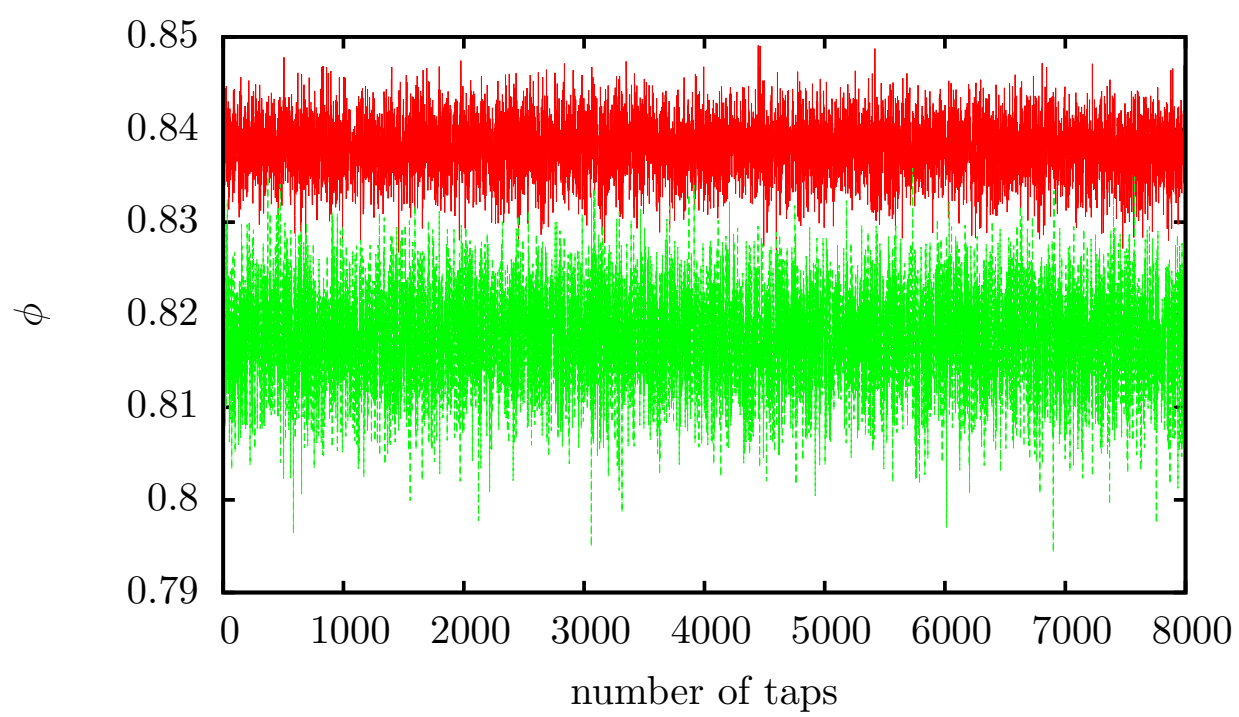

Fig. 4.1: Evolution of the global packing fraction $\phi$ with number of taps. $\phi$ is computed by $V_{g} / V$, where $V_{g}$ is the total volume of discs, and $V$ is the total Voronoi volume.

In earlier fluidized bed experiments, the ageing effect due to a change in the frictional properties of the grains has been reported by Schröter et al. [2005]: the friction between grains decreases with collisions. This ageing effect is also observed in the newly-made 

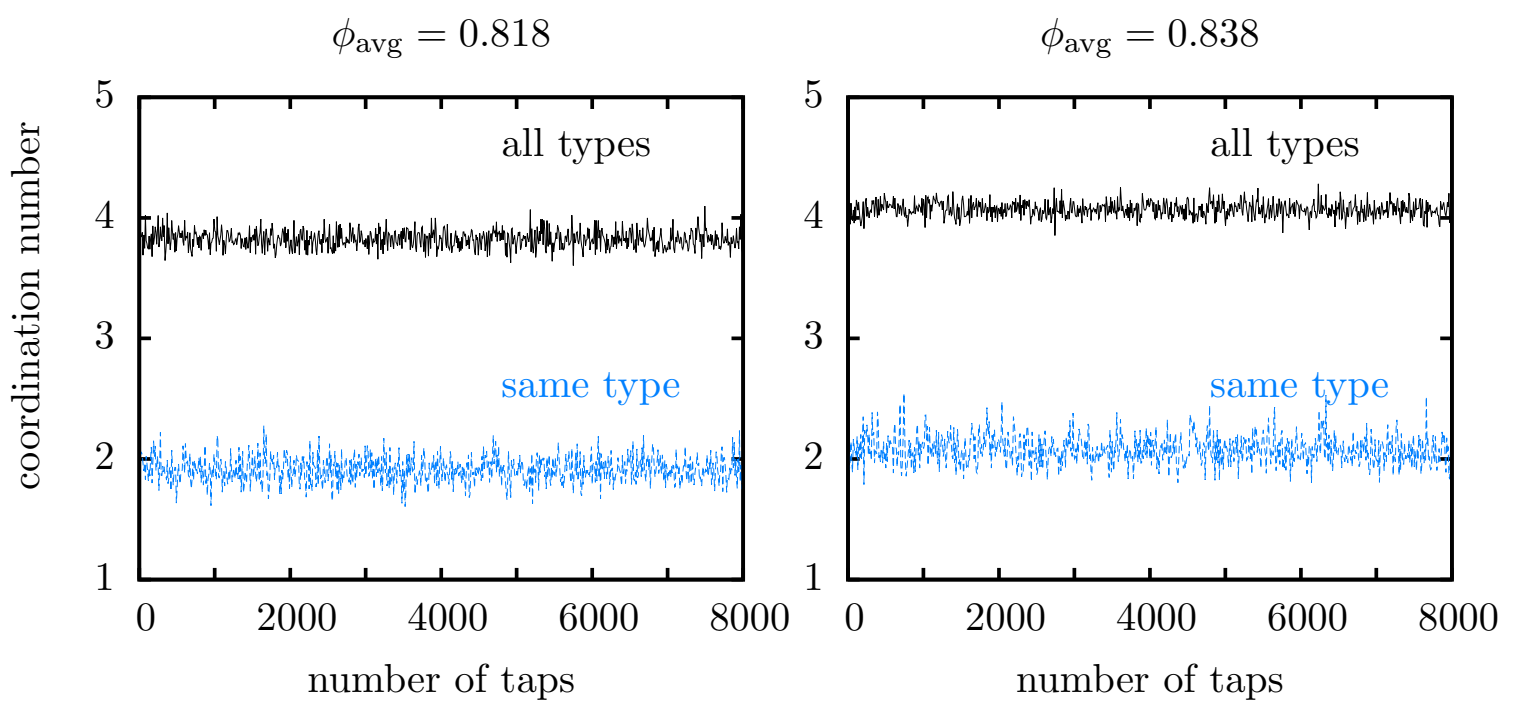

Fig. 4.2: Coordination number with number of taps. The "all types" coordination number counts all neighbours without distinguishing the disc type. While the "same type" coordination number only counts neighbours which are the same type as the central disk.

disc packings: packing fraction increases during the course of the experiment due to the decrease of the friction between grains. Before starting experimental measurement, the grains had been previously used for more than 20000 taps. The steady state is tested by looking at both global packing fraction (Fig. 4.1) and the average coordination number per grain (Fig. 4.2) over the course of each experiment.

Using the image processing explained in Chapter 3 the global packing fraction is computed as the total volume of the disks divided by the total Voronoi volume, $\phi=V_{g} / V$. The coordination number is computed by counting all neighbours within $1.1 \times\left(r_{c}+r_{n}\right)$, where $r_{c}$ is the radius of the considered disc and $r_{n}$ is the radius of the candidates. While 'all types' coordination number counts all neighbours, the 'same type' coordination number only counts the neighbours with the same type as the central disc, e.g small-small contacts. The same type contact number is very sensitive to the segregation. All data used for further analysis shows that no ageing and no segregation occurs during the course of the experiment.

The steady packing fraction and the steady contact number ensure that the system is stable with time. In addition our system is prepared under gravity. Sidewalls introduce slow convection rolls during an air flow pulse, and distributor inhomogeneities introduce gradients in the airflow. It is therefore not surprising to find gradients of the packing fraction in the system. The homogeneity about the local state should be verified as well.

The first question about the homogeneity of that cluster is the equality of the sampling of small and large discs. A cluster including $N$ particles is composed in the system. The total Voronoi volume and the number of small and large discs in that cluster are plotted with the cluster size $N$ in Fig. 4.3(a) and (b) respectively. The number of both small and large discs grow linearly with the cluster size $N$. The slope indicates the density. As one might expect small discs have a slightly higher density than the large discs.

To quantify the spatial and temporal variance the local density map is computed. Each 

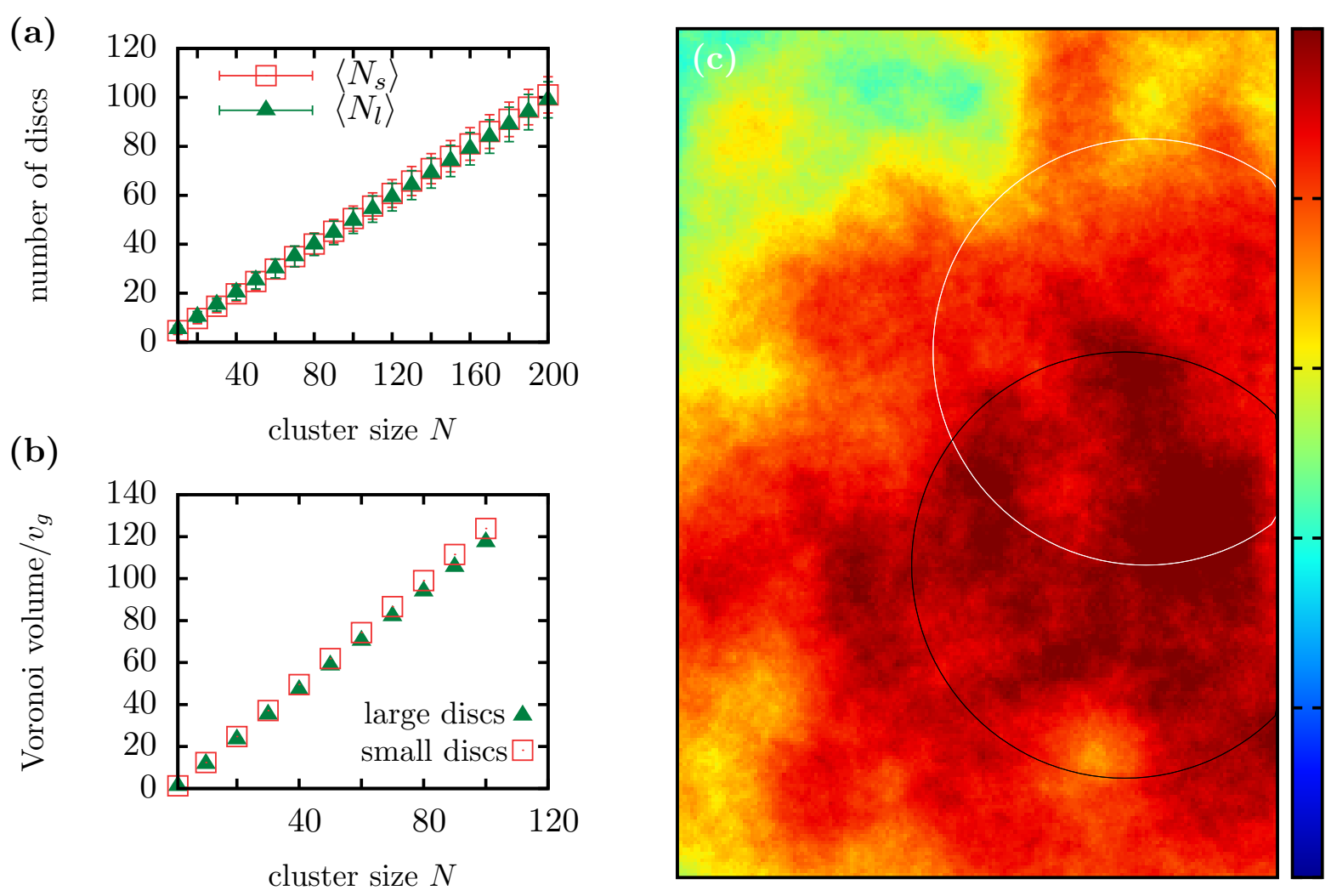

Fig. 4.3: (a): The number of small and large discs in a cluster with the cluster size for $\phi_{\text {avg }}=0.8380$. The angled brackets $\langle\ldots\rangle$ denotes averaging over 8000 images. (b): The total Voronoi volume of small and large discs with cluster size $N$ for $\phi_{\text {avg }}=0.8380$. (c): The map of local packing fractions averaged over 8000 images. The spatial resolution is 5 pixels $\sim 0.14 d_{s}$. The white circle denotes the most homogeneous region for this map, and has a diameter $18 d_{s}$.

point in the density map [cf. Fig. 4.3(c)] is computed using the 10 Voronoi cells nearest to that point To reduce the computation time and still get enough information, the spatial resolution of the density map is set to be 5 pixels and each density map is averaged over 10 random images, results in which 800 density maps for each experimental run. Over the 800 density maps the spatial variance is calculated in a spatially fixed circular region with diameter as $18 d_{s}$. By moving the center of that circular region through the density map a most homogeneous region is selected for further analysis [cf. Fig. 4.3(c)]. In all cases the variance in the analysis region is smaller than 0.00432 . It is clear by hindsight analysis that the homogeneity is essential to do quantitative studies on the correlation measurements, although the qualitative features are robust against the inhomogeneity.

We identify the most homogeneous region for every experiment, and perform various analysis inside those regions. We start with the distribution of volume distribution.

\footnotetext{
${ }^{1}$ The 'nearness' of a Voronoi cell to a given point is defined by the distance from that point to the surface of the particle which is included in that Voronoi cell. This definition considering the bidispersity is the extension of navigation map [Fig. 3.3 in chapter 3 . The nearness in a bidisperse system, of either Voronoi cells or particles will be defined like this from here on.

${ }^{2}$ The spatial variance of the whole system is about 0.0435 .
} 


\subsection{The histogram of Voronoi volume and free vol- ume}

The distribution of individual Voronoi volumes $v$ can be computed directly from the results of the navigation map. An example is shown in Fig. 4.4. The two peaks correspond to the distribution of the small and large discs respectively. The two distributions feature very similar shape, however, are separated by the intrinsic bidispersity of the disk volume. The difference of the two distributions is larger than their width.

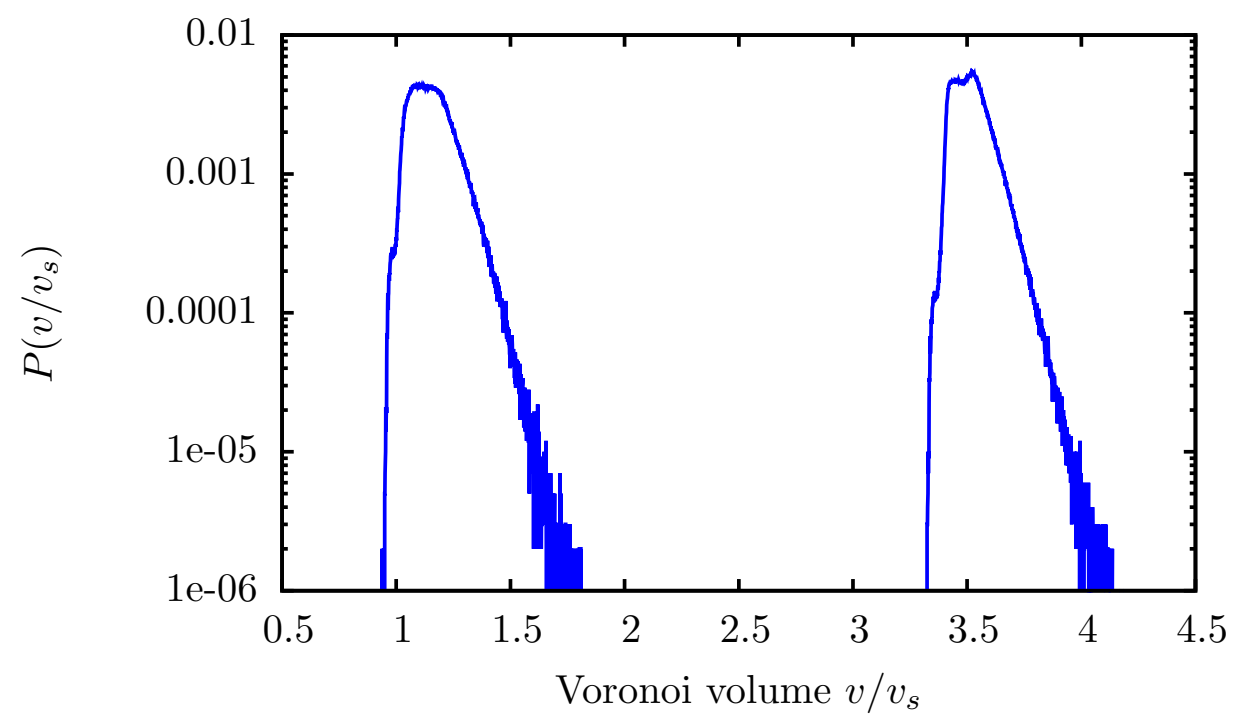

Fig. 4.4: Voronoi volume distribution. The two peaks corresponding to small and large discs respectively. Voronoi volume is rescaled by the volume of small discs $v_{s}$.

Because we do not want to distinguish between small and large discs, we follow Lechenault et al. [2006] and use in the subsequent analysis the free Voronoi volume $v_{f}=v-v_{\min }$. The minimum volume $v_{\min }$ is the volume that a grain would occupy in a hexagonal packing of identical discs. It equals $2 \sqrt{3} r_{g}^{2}$, where $r_{g}$ is the radius of the disc.

The free volume distributions of small and large discs and both types together are presented for two packing fractions in Fig. 4.5. Although the respective mean free volume of small and large discs still differ $\left(\bar{v}_{s}^{f}=0.135\right.$ and $\bar{v}_{l}^{f}=0.158$ in $v_{s}=\pi d_{s}^{2} / 4$, respectively, for $\left.\phi_{\mathrm{avg}}=0.8380\right)$, the difference now is much smaller than the width of the distributions. By using the free volume the success of the subsequent analysis justifies this step in hindsight.

For large volumes the decay is exponential but none of the free volume distributions and volume distributions in Fig. 4.4 and Fig. 4.5 could be fitted reasonably with a gamma distribution because of the slight binomial feature. This result differs from 2D and 3D mono-disperse packings Aste and Di Matteo, 2007, 2008, Kumar and Kumaran, 2005.

An interesting feature can be observed in Fig. 4.5. The relative height of the two peaks in the free volume distributions changes with $\phi_{\text {avg. }}$. A recent study of the probability distributions of quadron volumes (an alternative way of tessellation introduced in Blumenfeld 

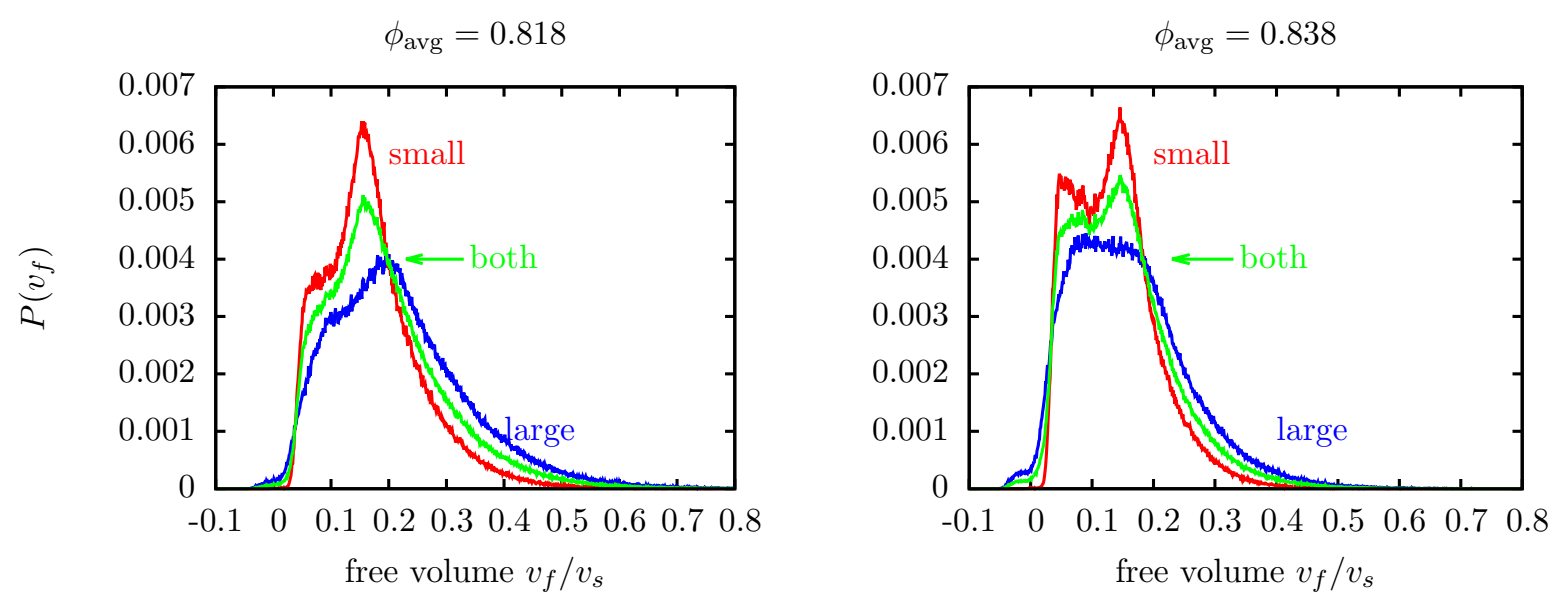

Fig. 4.5: Free volume distributions for $\phi_{\text {avg }}=0.818,0.838$. The red curves are the free volume distribution of small discs, the blue curves are of large discs, and the green curves are of both.

and Edwards, 2003]) showed that the position and height of these peaks can be traced to conditional probabilities of cell volumes at given coordination numbers Frenkel et al., 2008]. So the changes in the relative height of the two peaks visible in Fig. 4.5 may also be related to a change of contact number.

A compact cluster with size $N$ is defined as a central particle and its $N-1$ nearest neighbours as depicted in Fig. 4.6. The averaged free volume of a cluster composed of $N$ particles is defined as $v_{N}^{f}=V_{f} / N$, where $V_{f}$ is the total free volume of the cluster. The histograms of $v_{N}^{f}$ of compact clusters with different sizes $N$ are plotted in Fig. 4.6. The mean value, which is approximately the peak position is the same for different $N$, while the width or the standard deviation of the distribution, $\sigma_{N}^{f}$, decreases with $N$. As pointed out in Chapter 1, the exponent $\alpha \neq 1$ of this decreasing, $\sigma_{N}^{f} \sim N^{-\alpha / 2}$, indicates the existence of the correlation among the free volume of individual grains. We compute

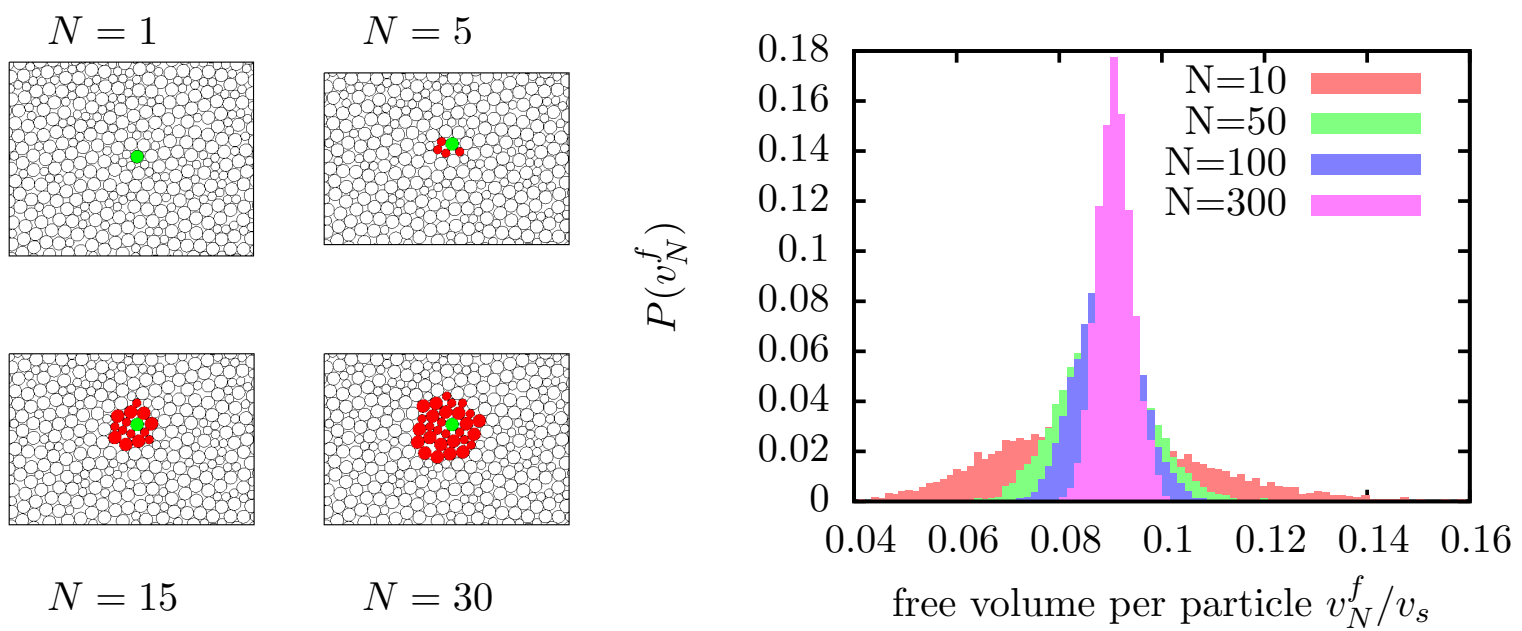

Fig. 4.6: LEFT: the construction of the compact clusters. The cluster is composed of a center particle (green) and its $N-1$ neighbours (red). RIGHT: the distribution of the free volume per particle with different cluster size $N$. 


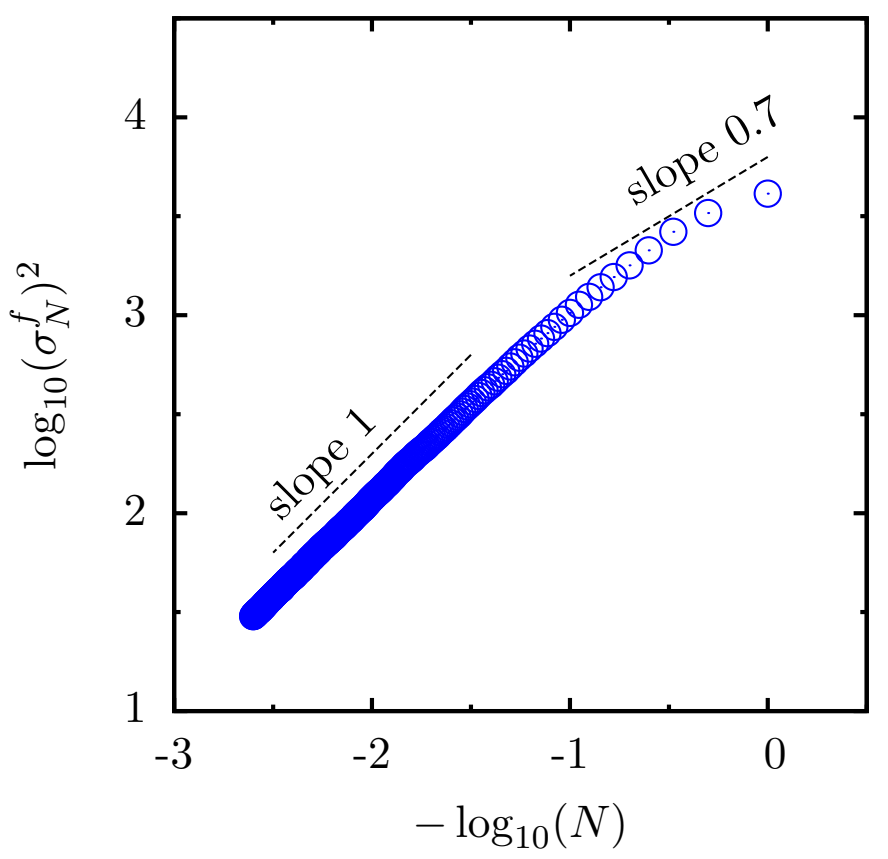

Fig. 4.7: The correlations have finite spatial extent, as shown by the variance $\sigma_{N}^{f}{ }^{2}$ of the average free volume of a compact cluster as a function of the number $N$ of grains included (open blue circles). While the slope for small clusters $(N<10)$ is 0.76 , in the large $N$ limit a slope one is recovered.

the variance $\sigma_{N}^{f}{ }^{2}$ of the distributions with different cluster size $N$. The results from $N=1$ to $N=300$ are plotted in Fig. 4.7 .

The small $N$ fitting has $\alpha \approx 0.76<1$. Nevertheless the difference is also obvious: the data points do not lie in a straight line or we can not fit the scaling as a single power law. The scaling seems to recover $\alpha=1$ with increasing $N$. The same behaviour is observed in 3D mono-sphere packing [Briscoe et al., 2008], but an earlier study on bi-disperse disk packings find $\alpha \approx 0.88$ even for $N>100$ [Lechenault et al., 2006]. This difference indicates that the quantitative detail of correlation might depend on the protocol. Henceforth in this chapter we only study the free volume, the subscript $f$ denoting free volume is omitted.

\subsection{Free volume of a cluster on a grid network}

The different scaling of the data points in Fig. 4.7 for small and large $N$ raises the expectation of the finite spatial extension of a correlation between Voronoi volumes. To measure this extension a cluster composed of spatially separated grains needs to be constructed rather than that a compact cluster [cf. Fig. 4.7].

A grid of evenly spaced points is imposed on the system. For each grid the nearest particle is chosen and the clusters contains only the grains on the grid [cf. Inset of Fig. 4.8]. By changing the spacing $L$ between grids, we can get various clusters formed by grains separated by different $L$. For the cluster on the grid with a given $L$ the variance of the 


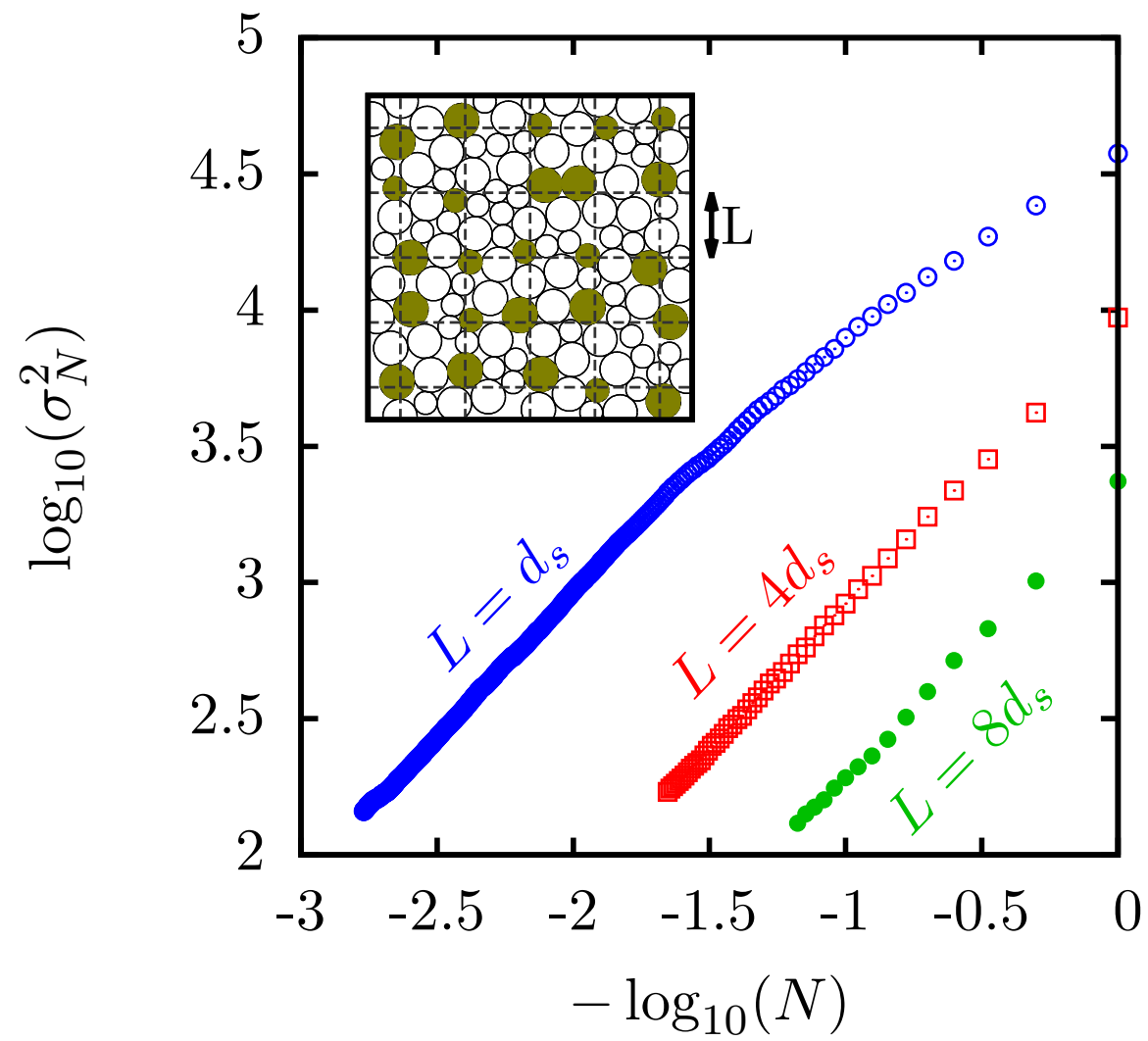

Fig. 4.8: INSET: A cluster constructed on a grid network with spacing L. MAIN PLOT: How $L$ affects the scaling of $\sigma_{N}^{2} \sim N^{-\alpha} . \sigma_{N}^{2}$ is the variance of the free volume per particle. $N$ is the number of particles in the cluster. Different colors correspond to different $L$ in the cluster. Data has been offset vertically for clarity. Text labels are rotated 45 degree to indicate the slope one. The large $N$ scaling are very similar between clusters with different $L$, parallel to each others, while the slope of small $N$ increases with $L$.

free volume per particle is computed for different cluster size $N$, just like that in Fig. 4.7 for a compact cluster. The dataset for $L=1,4,8 d_{s}$ are plotted in Fig. 4.8.

Although the slope of the large $N$ tail recovers to 1 in all three cases, the slope of small $N$ increases significantly from small spacing to large. More indicatively the scaling exponent $\alpha$ is measured by fitting a slope of the first five data points for each spacing $L$. Approximately

$$
\alpha \approx \frac{\log \left(\sigma_{N=5}^{2}\right)-\log \left(\sigma_{N=1}^{2}\right)}{\log \frac{1}{5}}
$$

The result is plotted in Fig. 4.9.

$\alpha$ indeed increases towards 1 with $L$ and appears to reach 1 at $L=4 d_{s}$. However the result is noisy and the definition of $\alpha$ is ambiguous: the number of data points used to extract $\alpha$ is arbitrary. One can extract $\alpha$ out of the first nine points, for example, rather than five. Qualitatively it is the same but quantitatively it is different. To quantitatively define an extension or a length one should first understand what $\alpha$ is measuring.

Consider a cluster including $N$ free volume of which each is denoted as $v_{i}$. The free volume per particle $\mathbb{V}_{N}=\frac{1}{N} \sum_{i=1}^{N} v_{i}$. Its mean value $\overline{\mathbb{V}}_{N}=\frac{1}{N} \sum_{i}\left\langle v_{i}\right\rangle$, where $\langle\ldots\rangle$ indicates 


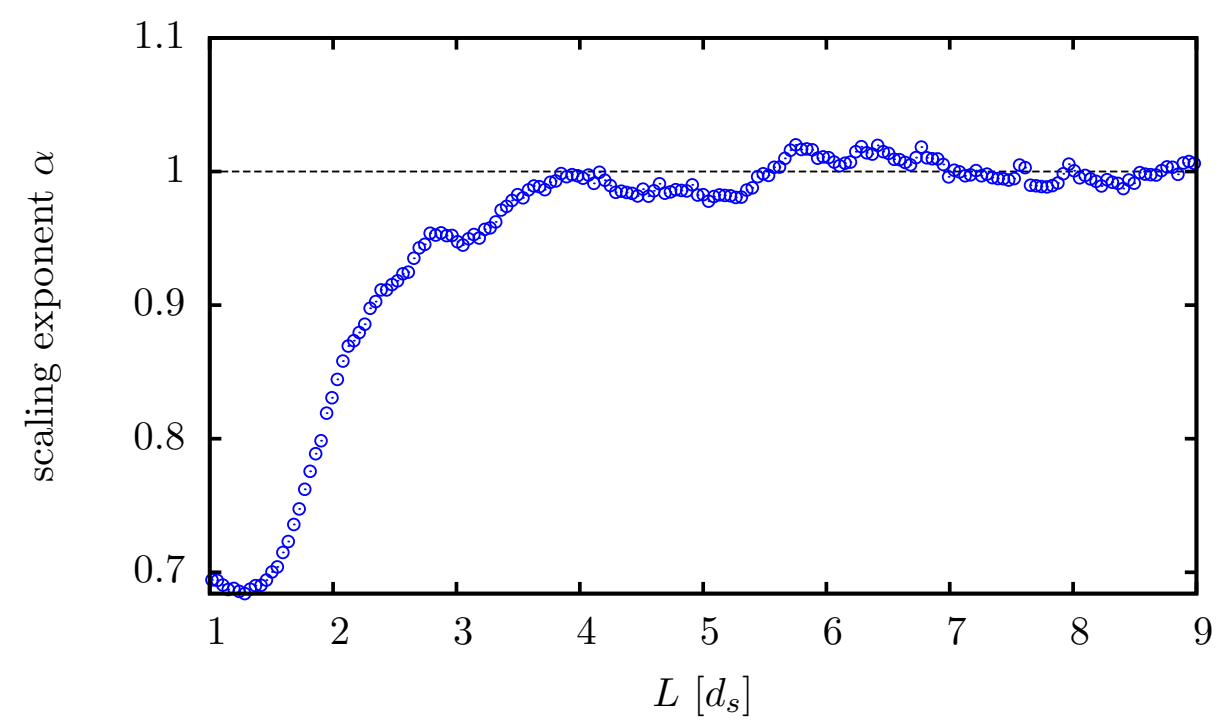

Fig. 4.9: The scaling exponent $\alpha$ measured by Eq. 4.1 on the cluster composed of grid network in Fig. 4.8 with spacing $L . L$ is normalized by the diameter of small discs $d_{s}$. The dip between $d_{s}$ and $2 d_{s}$ is due to the bidispersity.

averages over the 8000 images. The corresponding variance $\sigma_{N}^{2}=\frac{1}{N^{2}}\left\langle\left(\mathbb{V}_{N}-\overline{\mathbb{V}}_{N}\right)^{2}\right\rangle$ :

$$
\begin{aligned}
\sigma_{N}^{2} & =\frac{1}{N^{2}}\left\langle\left(\mathbb{V}_{N}-\overline{\mathbb{V}}_{N}\right)^{2}\right\rangle \\
& =\frac{1}{N^{2}}\left\langle\left[\sum_{i=1}^{N}\left(v_{i}-\bar{v}_{i}\right)\right]^{2}\right\rangle \\
& =\frac{1}{N^{2}}\left\langle\left[\sum_{i=1}^{N} \delta v_{i}\right]^{2}\right\rangle \\
& =\frac{1}{N^{2}}\left\langle\sum_{i=1}^{N} \delta v_{i} \sum_{j=1}^{N} \delta v_{j}\right\rangle \\
& =\frac{1}{N^{2}}\left(\sum_{i=1}^{N}\left\langle\delta v_{i}{ }^{2}\right\rangle+\sum_{\{i, j\}}\left\langle\delta v_{i} \delta v_{j}\right\rangle\right)
\end{aligned}
$$

where $\delta v_{i}$ denotes the fluctuation of free volume $i . \sum_{\{i, j\}}=\sum_{i=1}^{N} \sum_{j \neq i}^{N}$ sums over all pairs. $\left\langle\delta v_{i}{ }^{2}\right\rangle$ is the variance of individual free volume, and $\left\langle\delta v_{i} \delta v_{j}\right\rangle$ is the correlation between free volumes.

Following Eq. 4.2 the variance $\sigma_{N}^{2}$ can be decomposed into two parts. The first term in Eq. 4.2 is the sum of the variance of individual free volumes in the cluster. The second is the correlation between the fluctuation of free volumes in the cluster. Simply write 
$\left\langle\delta v_{i}{ }^{2}\right\rangle$ and $\left\langle\delta v_{i} \delta v_{j}\right\rangle$ as $\sigma_{i}^{2}$ and $C_{i j}$, then Eq. 4.2 is transformed into:

$$
\begin{aligned}
\sigma_{N}^{2} & =\frac{1}{N^{2}} \sum_{i=1}^{N} \sigma_{i}^{2}+\frac{1}{N^{2}} \sum_{\{i, j\}} C_{i j} \\
& =\frac{1}{N} \overline{\sigma^{2}}+\frac{1}{N^{2}} \sum_{i=1}^{N} C_{i}
\end{aligned}
$$

where $\overline{\sigma^{2}}=\sum_{i=1}^{N} \sigma_{i}^{2} / N$ is the average variance of individual free volume, and $C_{i}=$ $\sum_{j \neq i} C_{i j}$ is the correlation between free volume $i$ and other free volumes in the cluster. Now if the system is spatially homogeneous, which means that the variance of individual free volume $\sigma_{i}^{2}$ is independent of the subscript $i$, then $\overline{\sigma^{2}}$ is constant with $N$ and the first term is proportional to $1 / N$. Thus it is the second term, the correlation between free volumes, causing the scaling exponent $\alpha$ to be different from 1 . More explicitly, take the logarithm of Eq. 4.3 and substitute that into Eq. 4.1:

$$
\begin{aligned}
\alpha & =\frac{\log \sigma_{N}^{2}-\log \left(\bar{\sigma}^{2}\right)}{\log \frac{1}{N}} \\
& =1+\frac{\log \left[1+\sum_{i} C_{i} / N\right]}{\log \frac{1}{N}}
\end{aligned}
$$

From Eq. 4.4 one can construct two cases in which $\alpha=1$ would be recovered. The first case, very trivial, is that $C_{i}=0$, i.e. there is no correlation in the system, then Eq. 4.3 is reduced to

$$
\sigma_{N}^{2}=\frac{1}{N} \bar{\sigma}^{2}
$$

The second case is when the cluster size is much larger than the extension of the correlation $L_{\text {corr }}$. The free volumes which are closer to $i$ than $L_{\text {corr }}$ are correlated to the particle $i$ and contribute to $C_{i}$. When particle $i$ has all its correlated neighbours in the cluster, $C_{i}=\bar{C}$. With cluster size $N \gg L_{\text {corr }}^{2}$ almost every $C_{i}$ equals $\bar{C}$, except a small fraction of free volumes near the boundary. Thus the averaged contribution to correlation per particle $\frac{1}{N} \sum_{i} C_{i} \approx \bar{C}$ is approximately constant with $N$. Substitute this approximation into Eq. 4.3. For large cluster one can have

$$
\sigma_{N}^{2} \approx \frac{1}{N}\left(\overline{\sigma^{2}}+\bar{C}\right),\left(N \gg L_{\text {corr }}^{2}\right)
$$

Since both $\bar{\sigma}^{2}$ and $\bar{C}$ is constant, the extensive scaling is also recovered.

Both cases can be observed in Fig. 4.8. Eq. 4.5 indicates the happening of decorrelation, $C_{i}=0$, beyond a certain distance proved by the $L=8 d_{s}$ cluster in Fig. 4.8. Eq. 4.6 explains the large $N$ behaviour of $L=d_{s}$ [Fig. 4.8 and that of compact clusters [Fig. 4.7].

It should be emphasised that the two cases are quantitatively different due to the contribution of correlation $\bar{C}$, even though both recover $\alpha=1$. This difference could lead to some consequences on the statistical model of granular system. This topic will be discussed in Chapter 5 .

Eq. 4.4 not only connects the scaling exponent $\alpha$ with the correlation between Voronoi volumes explicitly, but also gives insight about the issue that might be encountered during 

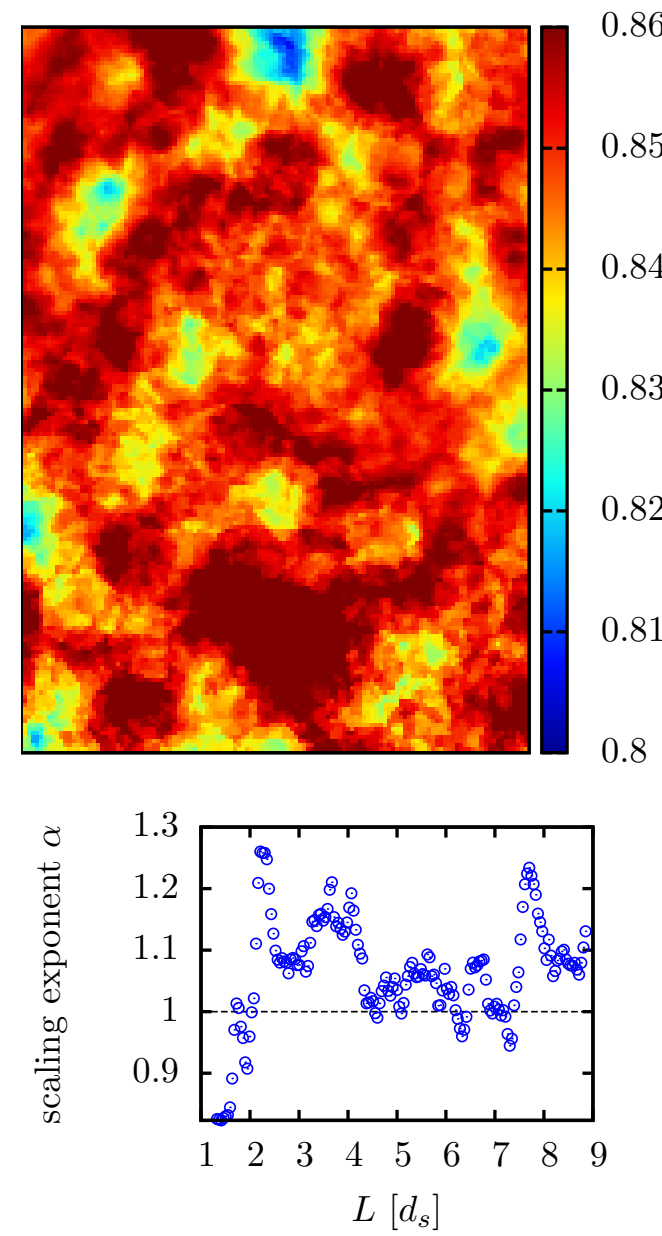
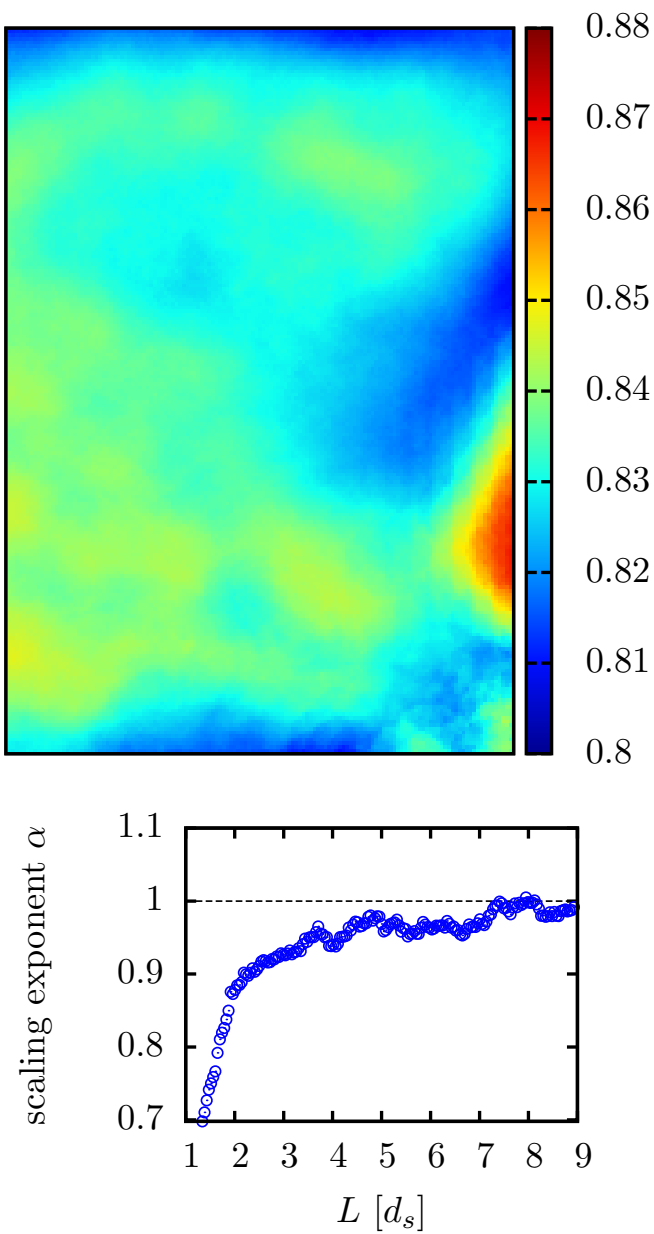

Fig. 4.10: Density maps and the corresponding scaling exponent $\alpha$ measurement. The density maps are computed in the same way as that in Fig. 4.3(c). LEFT COLUMN exhibits a experiment with large spatial variance resulting in fluctuation of $\alpha$ measurement. RIGHT COLUMN exhibits a experiment with a dense region (red triangle region at the right boundary). The corresponding $\alpha$ does not recover to 1 .

the measurement. For example, by carefully comparing Eq. 4.1 and Eq. 4.4 one could see that the averaged variance $\overline{\sigma^{2}}$ is used instead of that of a single particle $\left(\sigma_{N=1}^{f}\right)^{2}$. In a homogeneous system these two quantities are equal. In reality the packing could not be perfectly homogeneous. The inhomogeneity of local state induces noise to the measurement. The result in Fig. 4.9 suffers this effect. More severe situations are exhibited in Fig. 4.10. On the other hand the averaged correlation contribution $\bar{C}=\frac{1}{N} \sum_{i} C_{i}$ increases from 0 at $N=1$ to some constant at large $N$. According to Eq. 4.4 this makes $\alpha$ a function of the number of data points to fit (obvious in Fig. 4.7). Furthermore $\bar{C}$ counts correlations between all particles not only that from a given distance. This is the ambiguity of $\alpha$ mentioned following Eq. 4.1.

Due to the above two major disadvantages the direct measurement of correlation at a given distance is required to study the extension quantitatively. In the next section the grid cluster method is modified to serve this purpose. 

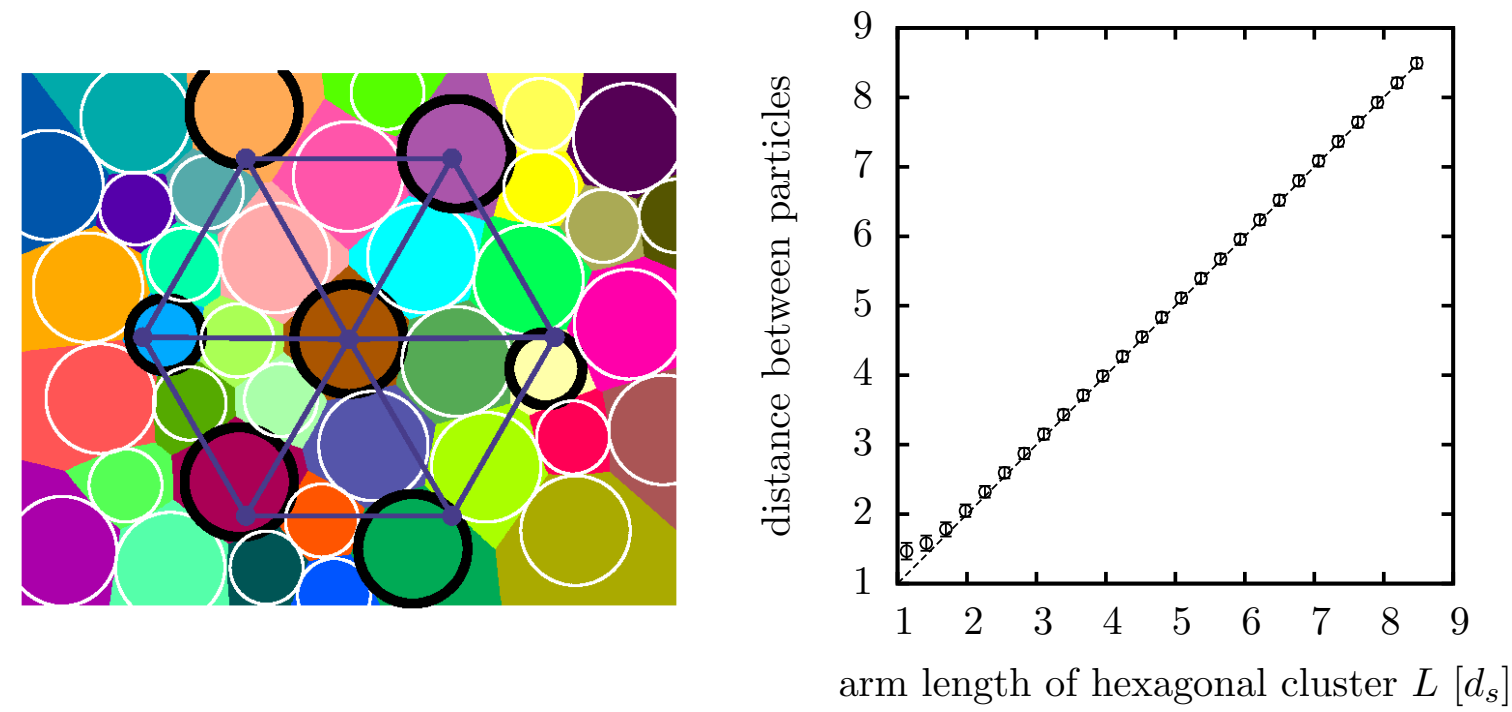

Fig. 4.11: LEFT: a hexagonal cluster in the packing. The side length of the hexagonal wheel is $L$. The six outer vertices and the center lie in seven different Voronoi cells. The particles corresponding to the seven Voronoi cells are highlighted by black circles. These particles are referred to as 'on the vertex' in the text. RIGHT: The actual distance between the centroids of particles on the vertex is computed for different $L$. The slope of the dashed line is 1 .

\subsection{Two-point correlation of free Voronoi volume}

To quantify the correlations between Voronoi volumes we use the two-point correlation function:

$$
C_{i j}(L)=\frac{\left\langle\left(v_{i}-\bar{v}_{i}\right)\left(v_{j}-\bar{v}_{j}\right)\right\rangle}{\sigma^{2}}
$$

where $i, j$ correspond to two points in a distance $L$ belonging to two Voronoi cells, and $v_{i}, v_{j}$ are the free volume of these Voronoi cells. $\langle\ldots\rangle$ indicates averages over all the 8000 different packings created by flow pulses. $\bar{v}_{i}$ and $\bar{v}_{j}$ are the mean free volumes at these points (computed separately to remedy the effect of remaining small gradients), and $\sigma^{2}=\left(\sigma_{i}^{2}+\sigma_{j}^{2}\right) / 2$ is the corresponding variance.

To obtain better statistics, 240 pairs of points with the same $L$ are selected. For each pair $C_{i j}(L)$ is computed using Eq. 4.7. In practice the pairs are selected in the following way: first a hexagonal "wheel" centred in the analysis region is constructed. The length of each edge of the wheel is set to be $L$ [c.f. Fig. 4.11]. The six outer points and the center form 12 pairs that are separated by a distance $L$. The physical distance between the two particles in each pair fluctuates around $L$. The mean value and the variance of the pair length are computed to make sure $L$ represents the physical distance on average [cf. Fig. 4.11.

For each such pair, the free volume of the two Voronoi cells to which the two vertices belong are taken as $v_{i}$ and $v_{j}$ in Eq. 4.7. Then the hexagon is translated to five different positions and rotated to four different angles for each position. However, the whole wheel stays inside the analysis region [cf. white circle in Fig. 4.3(c)]. Then the 240 pairs are averaged: 


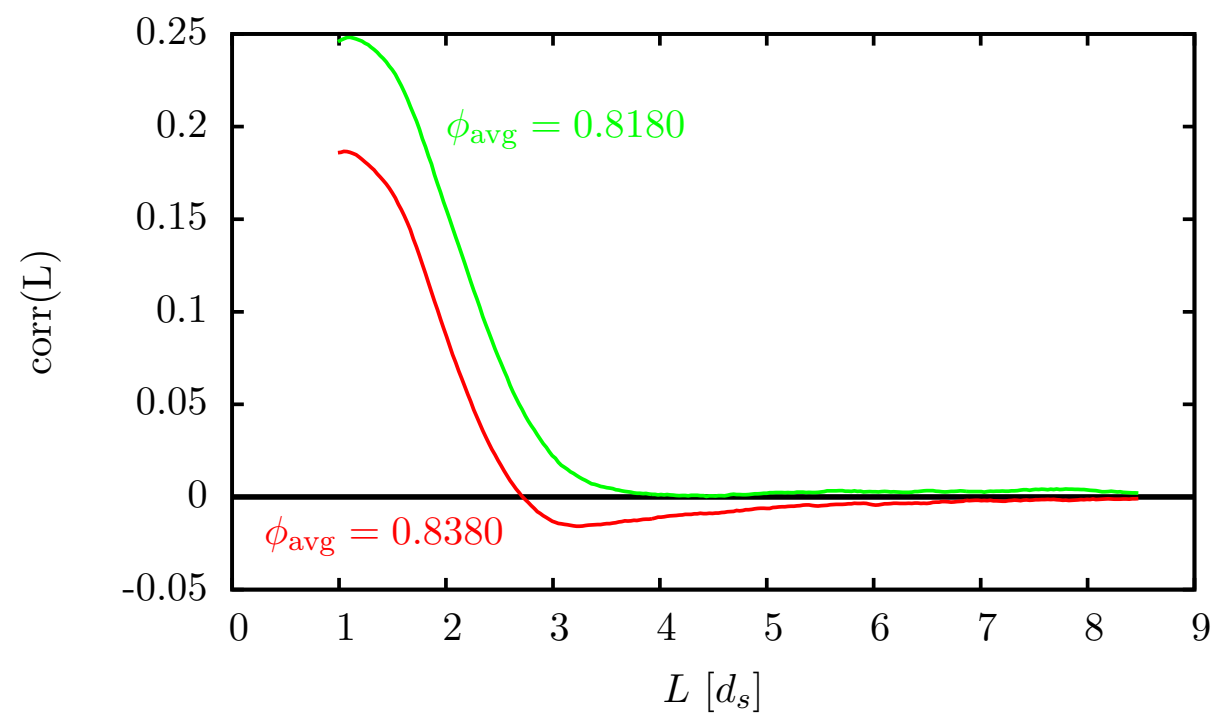

Fig. 4.12: Depending on $\phi, \operatorname{corr}(L)$ exhibits both correlations and anti-correlations.

$$
\operatorname{corr}(L)=\frac{1}{240} \sum_{i, j} C_{i j}(L)
$$

The correlation function $\operatorname{corr}(L)$ is shown for two values of $\phi_{\text {avg }}$ in Fig. 4.12. For low $\phi_{\text {avg }}, \operatorname{corr}(L)$ decays to zero and then fluctuates around it. Positive values of $\operatorname{corr}(L)$ indicate that the two free Voronoi volumes deviate from the average in the same direction. For high $\phi_{\text {avg }}, \operatorname{corr}(L)$ decreases to a negative minimum and then increases towards 0 . Negative values of $\operatorname{corr}(L)$ characterize anti-correlations: Voronoi cells at this distance deviate in opposite directions from the average free volume.

Anti-correlation indicates the competition for the free space between particles. One possible source of the anti-correlation is the remaining difference between the free volume distribution of small and large discs [cf. Fig. 4.5. Large discs have larger free volume than small discs on average. So by sampling the configuration that large and small discs are placed alternatively could introduce such anti-correlation feature. To clarify the nature of the observed anti-correlation Eq. 4.8 is performed on a hexagon cluster composed of only one type, small or large discs respectively [cf. Fig. 4.13]. Although $\operatorname{corr}(L)$ of one type discs is different from that of all type discs quantitatively, the anti-correlation exists in both case. Sampling only one type discs blurs $L$ from the actual distance of each pair even more. The correlation is also measured in a region with higher spatial variance (the dashed circle in Fig. 4.3(c)). The anti-correlation feature does not depend on the choice of the analysis region as well.

Gravity breaks the isotropy in our experimental setup; this anisotropy is visible in the correlations plotted in Fig. 4.14, where pairs of horizontal and vertical points are averaged separately. While the qualitative features of $\operatorname{corr}(L)$ are independent of direction, the anti-correlation along the gravity direction is stronger and extends to a larger length.

It is helpful to emphasis that the evolution of $\operatorname{corr}(L)$ with $L$ is different from the pair correlation function $g(r)$. The information at each $r$ of $g(r)$ enumerates the number of particles within the shell between $r$ and $r+\mathrm{d} r$. Different $r$ has different enumeration, so 


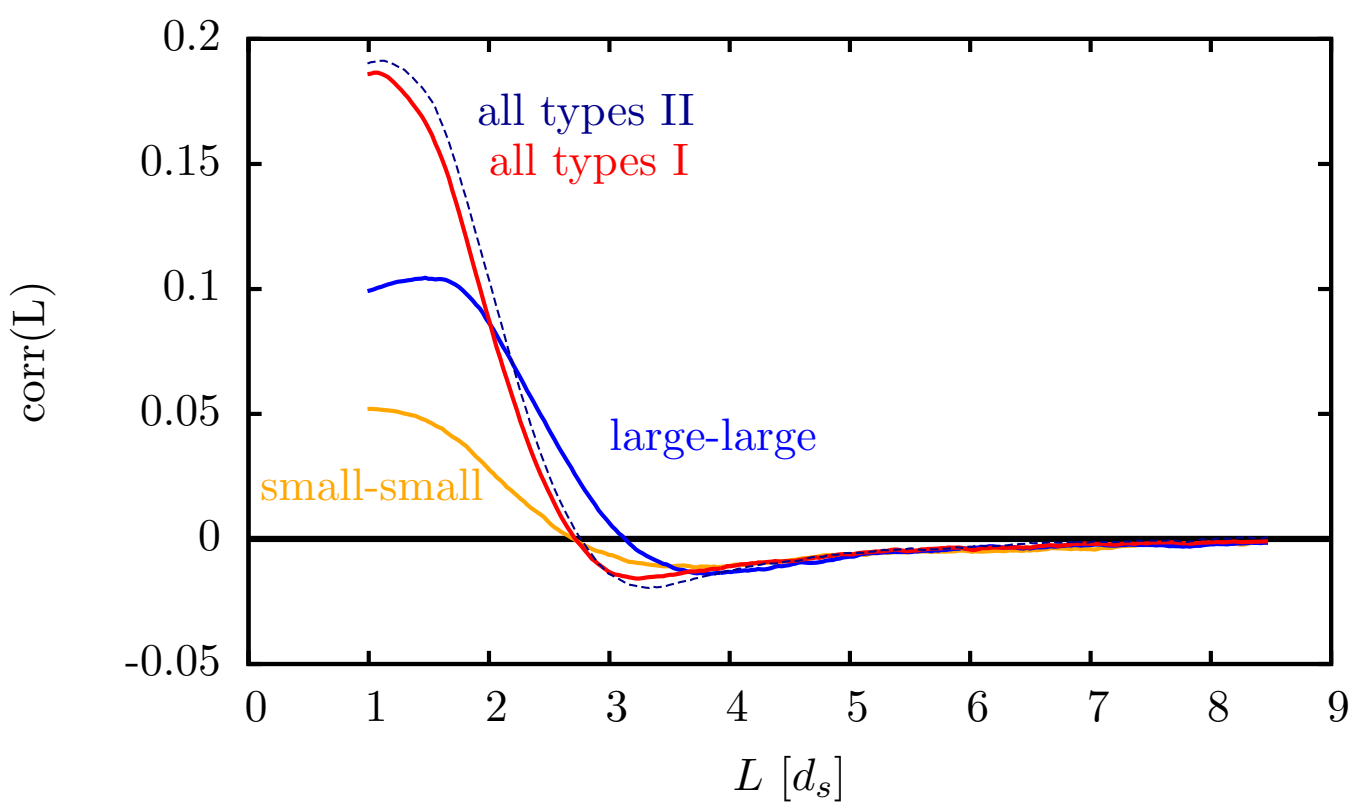

Fig. 4.13: the correlation measured between one type discs (small-small and large-large) and all types at $\phi_{\text {avg }}=0.8380$. The all type $I$ curve is the same as that in Fig. 4.12 . The all type II curve is measured in the dashed circle region in Fig. 4.3(c).

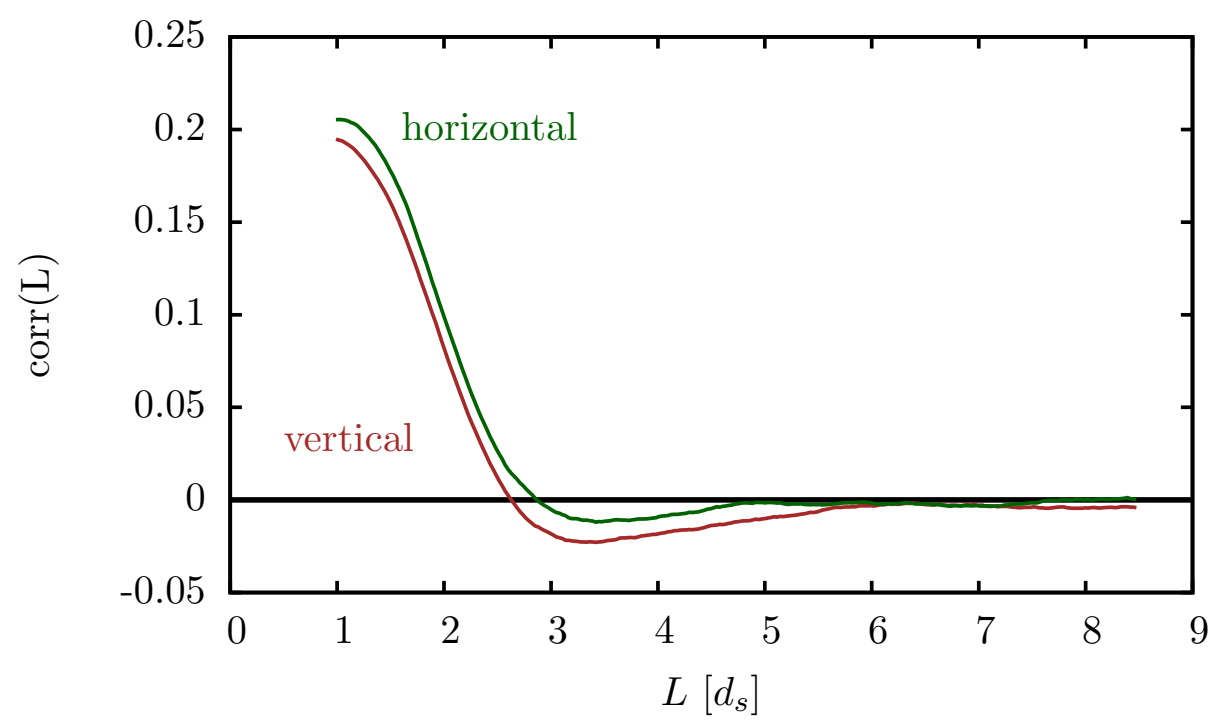

Fig. 4.14: the anisotropy measurement for $\phi_{\mathrm{avg}}=0.8380$. The two point correlation of pairs placed horizontally (dark green) and vertically (brown), rather than constructed from the hexagon. 
$g(r)$ has peak structure. The pair used in the correlation measurement always counts the same amount of particles no matter what value $L$ is by allowing the pair length fluctuate around $L$. So the statistics of each point of $\operatorname{corr}(L)$ is the same, and $L$ could still represent the actual pair length reasonably [cf. Fig. 4.11]. This is the advantage of using $L$ over $g(r)$ to define the pair length.

\subsection{Characteristic lengths of the correlation}

We define three characteristic lengths to describe $\operatorname{corr}(L)$ (see Fig. 4.15). First we do a linear fit to $\operatorname{corr}(L)$ for a range centred at half of the maximum of $\operatorname{corr}(L)$ with width $\pm 1 / 2 d_{s}$. The point where this fit crosses zero yields the length $L_{C}$. For measurements of $\operatorname{corr}(L)$ showing anti-correlation we define a second length, $L_{m i n}$, which is extracted from a local parabolic fit around the minimum of $\operatorname{corr}(L)$. The third length, $L_{A C}$, is obtained from an exponential fit ranging from $L_{\text {min }}$ to the end of $\operatorname{corr}(L)$ (we allow for a small offset in this fit, but the magnitude of this offset is less than 0.0006 in all cases).

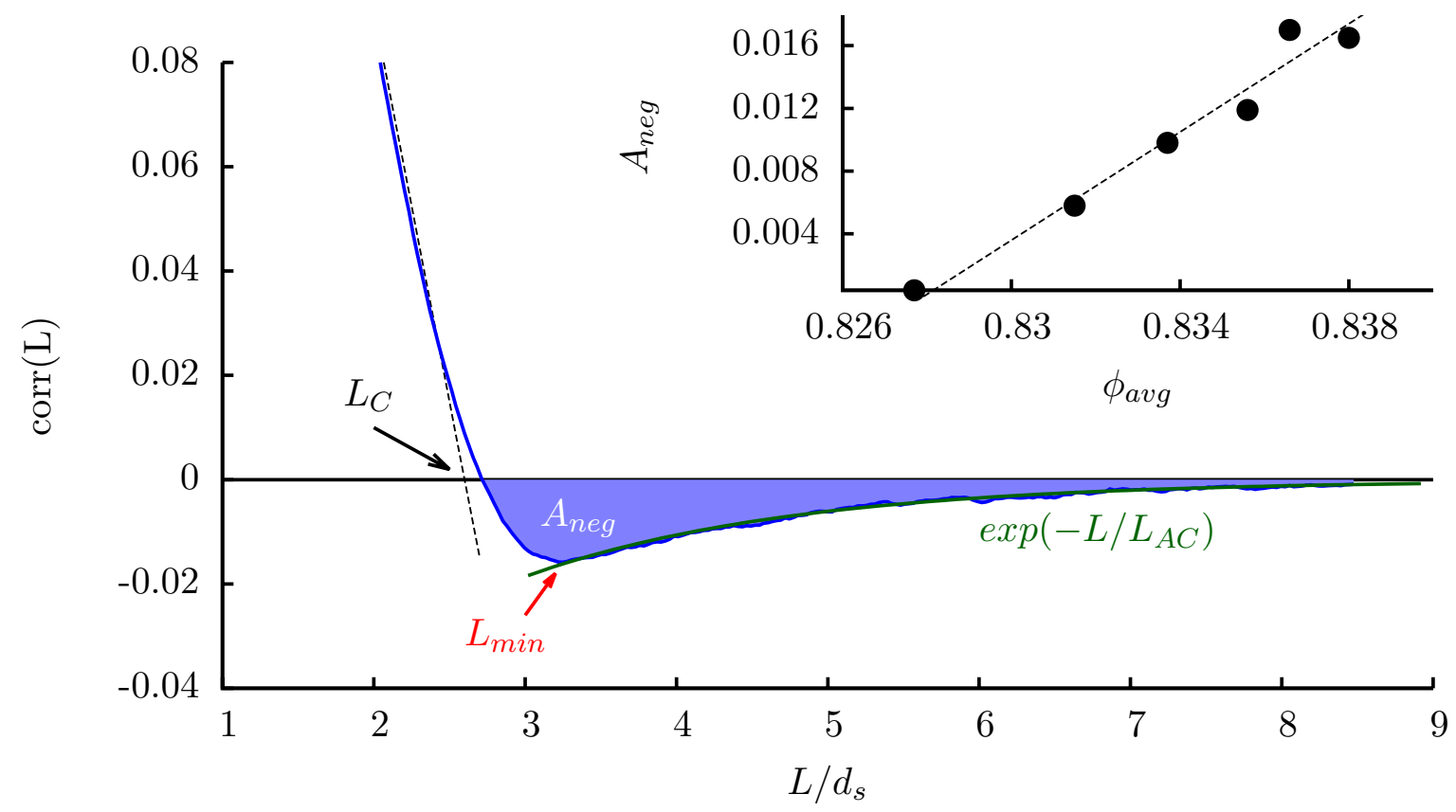

Fig. 4.15: Three characteristic lengths: $L_{C}$, obtained from a linear fit (dashed black line) to $\operatorname{corr}(L)$ for small $L ; L_{m i n}$, corresponding to the minimum of $\operatorname{corr}(L)$; and $L_{A C}$, obtained from an exponential fit (green line) to $\operatorname{corr}(L)$ for $L>L_{m i n}$. The inset shows the negative area $A_{n e g}$ of $\operatorname{corr}(L)$ as a function of of $\phi_{\text {avg }}$.

To find the onset of the anti-correlations, the area $A_{\text {neg }}$ where $\operatorname{corr}(L)<0$ [cf. Fig. 4.15 is plotted as a function of $\phi_{\text {avo }}{ }^{3}$, as shown in inset of Fig. 4.15. An extrapolation of a linear fit to $A_{\text {neg }}$ to zero defines the threshold for anti-correlations, $\phi_{A C}=0.8277 \pm 0.0005$. For $\phi>\phi_{A C}, \operatorname{corr}(L)$ can be both positive and negative; therefore, it can not be described by a power law Lechenault et al., 2006].

\footnotetext{
${ }^{3}$ When computing $A_{\text {neg }}$ we take in to account the offset of the exponential fit determining $L_{A C}$.
} 


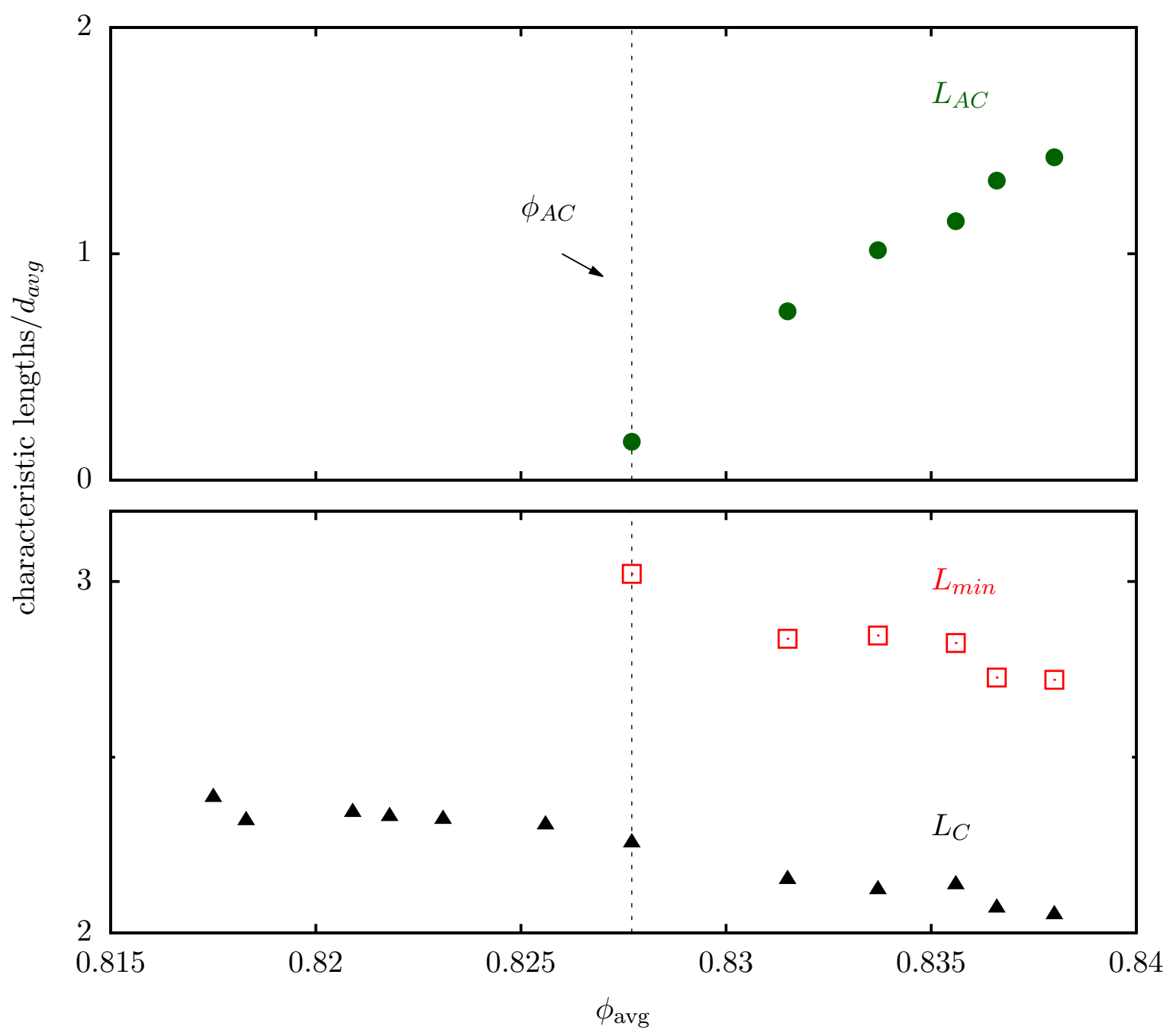

Fig. 4.16: Packing fraction dependence of the characteristic lengths of correlation $L_{C}$ and anticorrelation $L_{\min }$ and $L_{A C}$. The lengths are normalized using the average disc diameter $d_{\text {avg }}$, which corresponds to $1.23-1.28$ times $d_{s}$.

The dependence of $L_{C}, L_{m i n}$, and $L_{A C}$ on $\phi_{\text {avg }}$ is shown in Fig. 4.16, $L_{C}$ and $L_{\text {min }}$ slowly decrease monotonically with $\phi_{\text {avg }}$, while $L_{A C}$ grows approximately linearly. All lengths are normalized by the average disc diameter $d_{\text {avg }}=2 \sqrt{\frac{V_{g}}{N \pi}}$, where $V_{g}$ is the total volume of discs, and $N$ is the total number of discs in the system.

We try to estimate the error bar of the lengths using the following method. According to Eq. 4.7 the correlation is measured by averaging 8000 images. The same equation is performed on a set consisting of $n_{\text {ing }}$ images at a given $\phi_{\text {avg }}^{4}$. Out of 8000 images $8000 / n_{\text {img }}$ image-sets are composed and constitute an ensemble. For each image-set the correlation curve is measured and the lengths are extracted as above. The standard deviation of the lengths are computed in the image-set ensemble. For instance, four $\operatorname{corr}(L)$ can be computed for $n_{i m g}=2000$. Four $L_{C}$ can be extracted out of the four $\operatorname{corr}(L)$, and the variance is computed accordingly. The standard deviation of $L_{C}$ and $L_{A C}$ are plotted with

${ }^{4}$ The $n_{\text {img }}$ images in a dataset are not continuous. 


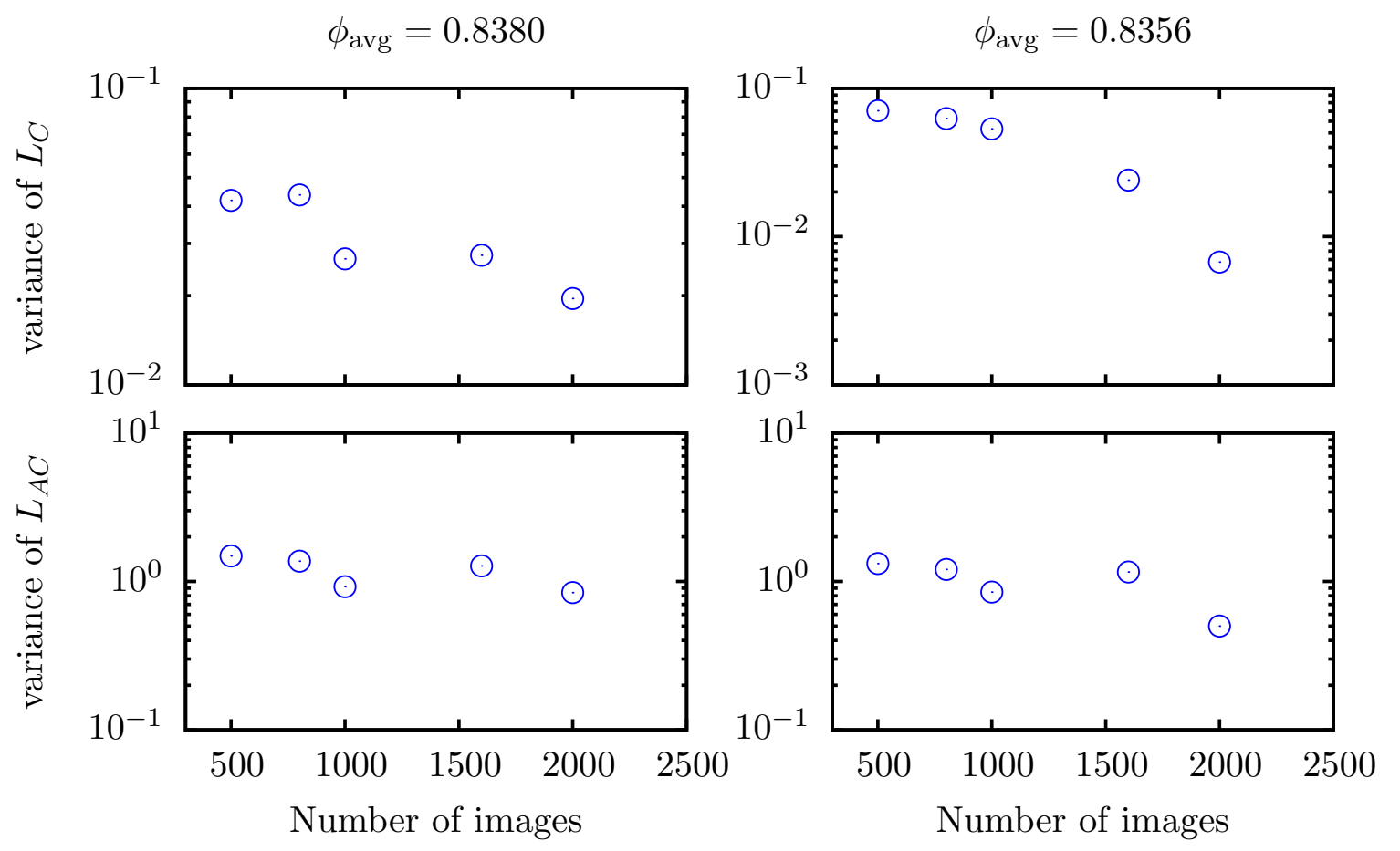

Fig. 4.17: The errorbar estimation of lengths $L_{C}, L_{A C}$ for $\phi_{\text {avg }}=0.8356,0.8380$. The lengths are extracted from $\operatorname{corr}(L)$ point is computed by averaging the corresponding number of images $n_{\text {img }}=500,800,1000,1600,2000$ using Eq. 4.7. The data points are the variance of the lengths. A straight line is fitted for each dataset in logarithm scale and is extrapolated to $n_{\text {img }}=8000$ to get the corresponding error bar. The computation of the variance is explained in the text.

$n_{i m g}$ for two $\phi_{\text {avg }}$ in Fig. 4.17. As $n_{i m g}$ increases the standard deviation of the lengths decreases. The data points are fitted and extrapolated to $n_{i m g}=8000$ in logarithm scale to get the estimation about the error bar. The computed error bar are listed in Tab. 4.1.

The range of $L_{C}$ is $0.55 d_{s}$, and its error bar is less than $6 \%$ of this range on average. $L_{\text {min }}$ has a slightly larger range $0.5 d_{s}$, and almost all error bars are below $0.8 \%$ of its range except that of $\phi_{a v g}=\phi_{A C} . L_{A C}$ varies over $1.56 d_{s}$, and the corresponding error bar is around $30 \%$ of this range. Similar to $L_{\min }$ the two packings near $\phi_{A C}$ have larger error bars. 
Tab. 4.1: The lengths and their error bar in unit of the diameter of small discs $d_{s}$. The error bar is estimated by extrapolating the standard deviation of the lengths from small image sets to the whole 8000 images. The details of the method is described in the text and Fig. 4.17.

\begin{tabular}{|cccc|}
\hline$\phi_{\mathrm{avg}}$ & $L_{C}$ & $L_{\min }$ & $L_{A C}$ \\
\hline 0.8277 & $2.798 \pm 0.007$ & $3.732 \pm 0.170$ & $0.209 \pm 0.404$ \\
0.8315 & $2.676 \pm 0.018$ & $3.361 \pm 0.003$ & $0.915 \pm 0.460$ \\
0.8337 & $2.717 \pm 0.012$ & $3.472 \pm 0.004$ & $1.278 \pm 0.260$ \\
0.8356 & $2.667 \pm 0.001$ & $3.331 \pm 0.002$ & $1.412 \pm 0.280$ \\
0.8366 & $2.625 \pm 0.022$ & $3.318 \pm 0.004$ & $1.654 \pm 0.331$ \\
0.8380 & $2.602 \pm 0.012$ & $3.237 \pm 0.004$ & $1.772 \pm 0.260$ \\
\hline
\end{tabular}

\subsection{Discussions}

This is the first observation of anti-correlation between Voronoi volumes in a granular system. One reason for this is that earlier studies of volume fluctuations focused on the cluster composed of neighbouring particles Aste and Di Matteo, 2007, Lechenault et al., 2006]. There the variance of average free volume per grain $\sigma_{N}^{2}$ as a function of the number of grains included $N$ was measured, and the scaling $\sigma_{N}^{2} \sim N^{-\alpha}$ with $\alpha<1$ indicated the existence of correlations. However, the scaling between $\sigma_{N}^{2}$ and $N$ is a measure of the correlation integrated over the whole cluster size. In the large $N$ limit, the relation between $N^{-\alpha}$ and $\operatorname{corr}(L)$ is:

$$
N^{-\alpha}=\frac{\mathcal{C}}{N} \int_{0}^{L(N)} \operatorname{corr}(L) L \mathrm{dL}
$$

where $\mathcal{C}$ is a constant proportional to the density of the cluster $\left(\pi L(N)^{2}\right.$ is the size of the cluster). The relatively small contribution of anti-correlations to the integral in Eq. 4.9 would be hard to distinguish from experimental noise [cf. Fig. 4.7]. Therefore, a direct measurement of two-point correlation is necessary to find anti-correlations.

A statistical mechanics approach for granular systems has been developed where the Hamiltonian is replaced by a volume function [Edwards and Oakeshott, 1989]. Voronoi cells have been used to construct this function [Aste and Di Matteo, 2008, Lechenault et al. 2006, Song et al., 2008]. While the discovery of anti-correlation has consequences for such an approach, some properties like the distribution of the local packing fraction have been shown to not depend on correlations [Puckett et al., 2011].

It is possible that not only the extent but even the existence of positive correlation is an artefact of Voronoi tessellation. By definition the Voronoi tessellation assigns space to each particle in a certain 'equal' way. In the case of the navigation map, the edge of a Voronoi cell is the collection of points that have equal distance $r$ to the surface of the neighbouring particles. The increase in a Voronoi volume could roughly be seen as the increase in $r$, therefore the $r$ of its neighbouring Voronoi cell. Consequently the Voronoi tessellation itself gives rise to a positive correlation between neighbouring Voronoi volumes. To verify whether this effect dominates the evolution of $L_{C}$, we estimate the average size $d_{\text {vor }}$ of Voronoi cells in the system in the following way. In a homogeneous 
system, $\phi_{\text {avg }}$ could be written as the ratio of the average particle volume to the average Voronoi volume, $\phi_{\mathrm{avg}}=d_{\mathrm{avg}}^{2} / d_{\mathrm{vor}}^{2}$. Given the bidisperse nature of our system $d_{\mathrm{avg}}$ is in the range of $1.23-1.28 d_{s}$. This estimation leaves us $d_{\mathrm{vor}}=d_{\text {avg }} / \sqrt{\phi_{\mathrm{avg}}}$. Rescaling $L_{C}$ with $d_{\text {vor }}$ shows that $L_{C}$ decays slightly faster than explained by simple compaction. This could stem from the appearance of the anti-correlation beyond $\phi_{A C}$. It will be interesting to test this hypothesis with another way of tessellation, such as quadrons Blumenfeld and Edwards, 2003.

Concerning the physical interpretation of these anti-correlations, we conjecture that $\phi_{A C}$ might correspond to the onset of dilatancy. In 3D systems it is well established that loose granular material collapses under shear, while granular material denser than dilatancy onset expands Gravish et al., 2010, Kabla and Senden, 2009]. This expansion can be understood as competition between the grains for free volume.

Our results show that correlations between the volumes of subunits depend on the specific system under consideration. For example, in froths there exist anti-correlations of the number of faces between neighbouring cells Hilhorst, 2008, Kumar et al., 1994, OD́onovan and Möbius, 2011, Oger et al. 1996]. At the same time the volume of a foam cell is proportional to the number of faces [Oger et al., 1996, Stoyan et al., 1995]. These features combined indicate that the volume of neighbouring cells should be anti-correlated.

Intuitively the anisotropy brings the connection with the force chain Majmudar and Behringer, 2005, where the spatial force correlation covers a much longer range along the force chain than perpendicular to it. In a jammed packing the contacts between particles bear forces. When a locally geometrical rearrangement happens which changes the Voronoi volume, the contacting forces are adapted for the new local configuration. This local rearrangement most likely propagates along the direction of the external applied force (in this case the gravity) to attain a lower energy state.

This anisotropy observation may raise the interests on measuring the correlation in the force or stress ensemble [Henkes and Chakraborty, 2009, Henkes et al., 2007]. In fact the anisotropy of the stress correlation has been observed. There a bidisperse disc packing is sheared by expanding in one direction and compressing in perpendicular direction at a fixed density just above jamming. For the simulation model the stress correlation along the expanded direction exhibit anti-correlation while that along the compressed direction only has positive correlation. Interestingly the photoelastic experiment performed in the same configuration does not show any anti-correlation and has a slow decay in along the compressed direction Lois et al. 2009]. On the other hand an alternative tessellation method, quadrons which considers the contact force into its cell construction is an candidate as well [Blumenfeld and Edwards, 2003, 2009].

It would be interesting to know how lengths would develop at wider range of packing fraction. However, we can not compactify the system above $\phi_{\text {avg }}=0.838$ without partial segregation. In this case no anti-correlations occur within the segregated patches. The only surviving length is $L_{C}$, which characterizes the size of the segregated patches. On the other hand, the evolution of correlation towards lower packing fraction will be discussed in the next section. 


\subsection{Further results and Outlook}

In this section we are going to show some preliminary results of another tessellation method, and another possible way to extract characteristic length scales from $\operatorname{corr}(L)$, and the evolution of $\operatorname{corr}(L)$ around random loose packing.

\subsubsection{Radical Tessellation vs. navigation map}

We have performed our analysis with the radical tessellation ${ }^{5}$ as well, and have found all features stay qualitatively the same. Quantitatively there are differences.
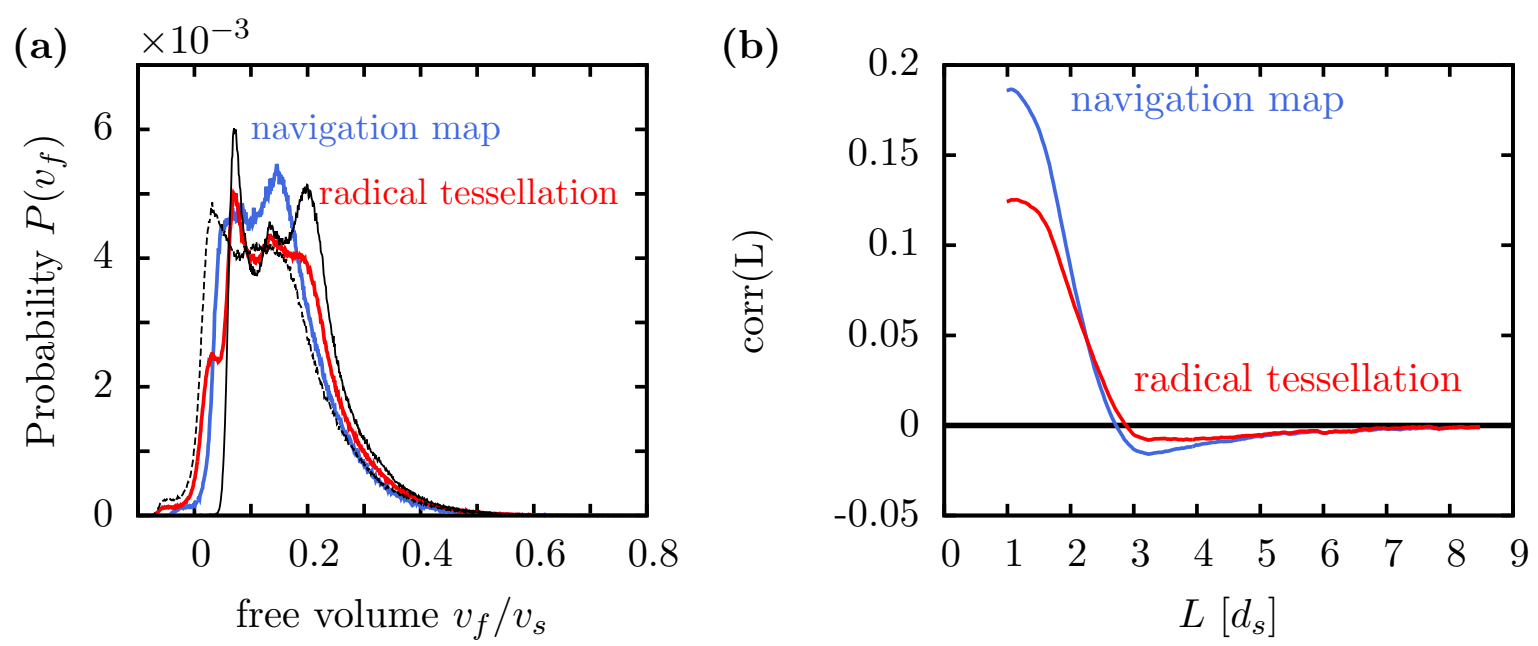

Fig. 4.18: (a): The free volume distributions of navigation map (blue) and radical tessellation (red) for $\phi_{\text {avg }}=0.8380$. The black solid and dash lines are the distribution of small and large discs for radical tessellation respectively. $v_{s}$ is the volume of small discs. (b): Correlation between free volume, $\operatorname{corr}(L)$, of both tessellation algorithms for $\phi_{\text {avg }}=0.8380 . d_{s}$ is the diameter of small discs.

Firstly, although the difference of peak positions is trivial, the free volume distribution for radical tessellation has more structures. The distribution of small discs and large discs are quite different in shape [black curves in Fig. 4.18(a)]. The position of peaks corresponds to some local configurations, e.g. the left peak corresponds to small-small local structure. This observation implies that radical tessellation is more sensitive to the local packing. With segregation the local packings are equivalent to monosize packings, where both navigation map and radical tessellation are reduced to the Voronoi tessellation. In the free volume distribution this is the regime near 0. As can be seen in Fig. 4.18(a) that the near 0 side of the distributions for navigation map and radical tessellation are still different, which indicates that no segregation happens in the packings.

The second difference is the $\operatorname{corr}(L)$ function. In Fig. 4.18(b) the $\operatorname{corr}(L)$ of the same experiment $\left(\phi_{\mathrm{avg}}=0.8380\right)$ with both tessellation methods are plotted. The maximum value of radical tessellation is smaller than that of navigation map. Nevertheless the

\footnotetext{
${ }^{5}$ Radical tessellation takes the edge of Voronoi cells as the collection of points which have the same tangent length to the neighbouring particles. See Chapter 3
} 
positive correlation persists to a longer distance, and the minimum negative value is larger than that of navigation map. The tails of the two $\operatorname{corr}(L)\left(L>6 d_{s}\right)$ overlap. All of these features make $\operatorname{corr}(L)$ of radical tessellation not as dramatic as that of navigation map.

We identify the onset of anti-correlation $\phi_{A C}$ by measuring the area below 0 for radical tessellation. The resulting $\phi_{A C}=0.828 \pm 0.002$ is larger than that on navigation map. Counting on the error bar the ranges of these two onset overlaps. Above this onset we extract the three lengths: $L_{C}, L_{\min }$ and $L_{A C}$ and list the result in Tab. 4.2. The quantitative difference of $L_{C}, L_{\min }$ and $L_{A C}$ are $6 \%, 12 \%$ and $30 \%$ larger on average.

Tab. 4.2: The lengths extracted from $\operatorname{corr}(L)$ measured on radical tessellation. For comparison each length is normalized by the corresponding one measured on navigation map.

\begin{tabular}{|cccc|}
\hline$\phi_{\mathrm{avg}}$ & $L_{C}$ & $L_{\min }$ & $L_{A C}$ \\
\hline 0.8315 & 1.053 & 1.155 & 1.394 \\
0.8337 & 1.096 & 1.141 & 1.066 \\
0.8356 & 1.087 & 1.148 & 0.844 \\
0.8366 & 1.094 & 1.113 & 1.381 \\
0.8380 & 1.063 & 1.104 & 1.599 \\
\hline
\end{tabular}

The free volume is introduced to cancel the bidispersity in the system. Nevertheless the remaining difference between the distribution of small and large discs for radical tessellation is larger than that for navigation map. This remaining difference induces the spatial correlation between the two types of discs into $\operatorname{corr}(L)$ measurement. Therefore the measurement based on radical tessellation is not compatible to reflect the correlation of the fluctuation of local volumes.

\subsubsection{Two exponential fit}

Above $\phi_{A C}$ the fluctuation of the volume of one particle causes more and more particles to be anti-correlated, which is indicated by the increase of $L_{A C}$ and the decreases of $L_{\text {min }}$. Also $L_{C}$ decreases more steeply above $\phi_{A C}$ [cf. Fig. 4.16. The three lengths are not independent. This observation raises the hypothesis that the anti-correlation and positive correlation co-exist in the system and compete with each other. As the packing becomes denser, the tail of the anti-correlation, $L_{A C}$, becomes longer, which indicates that the anti-correlation becomes stronger. Stronger anti-correlation weights more in the competition. Therefore the negative minimum characterized by $L_{m i n}$, where anticorrelation is observed strongest, happens at a shorter distance, and $L_{C}$, beyond which the anti-correlation overweights positive correlation, decreases.

This hypothesis could be formulated as Eq. 4.10. $d_{\text {avg }}$ is the average disc diameter. This fit includes five parameters: $A, B, l_{c}, l_{a c}$ and offset. $A$ and $B$ are the amplitude of positive and anti-correlation respectively. Both types of correlations decay exponentially when $L>d_{\mathrm{avg}} . l_{c}$ and $l_{a c}$ are the respective length scales.

$$
\operatorname{corr}(L)=A \exp ^{-\frac{L-d_{\text {avg }}}{l_{c}}}-B \exp ^{-\frac{L-d_{\text {avg }}}{l_{a c}}}+\text { offset }
$$



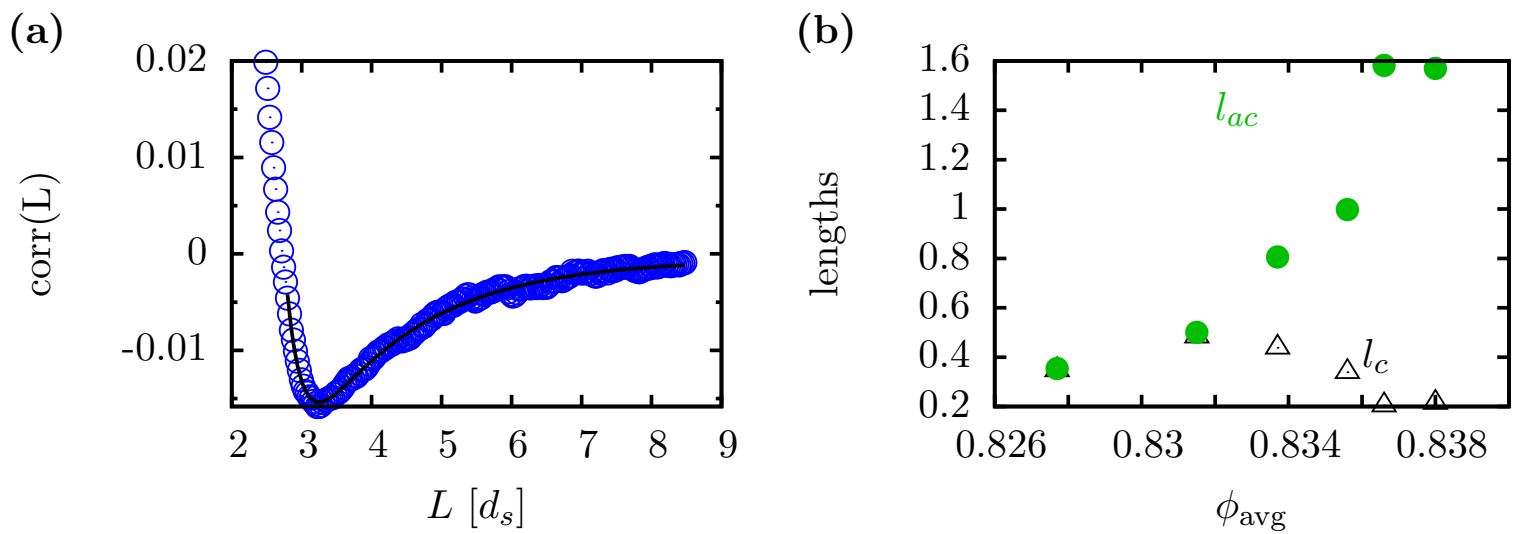

Fig. 4.19: (a): the correlation, $\operatorname{corr}(L)$, for $\phi_{\text {avg }}$ (blue points) and the corresponding two exponential fit (solid line) in Eq. 4.10. (b): The two length scales extracted from the two exponential fit, $l_{c}$ and $l_{a c}$. $l_{c}$ characterizes the positive correlation, while $l_{a c}$ characterizes the anti-correlation.

The correlation curves with $\phi_{\mathrm{avg}}>\phi_{A C}$ are fitted with Eq. 4.10. The fitting only covers the range where $L>2.5 d_{s}$ [cf. Fig. 4.19]. The offset of all fits are within \pm 0.0006 . $l_{c}$ and $l_{a c}$ are comparable and both increase till $\phi_{\text {avg }}=0.8330$. Above this packing fraction $l_{c}$ decreases, while $l_{a c}$ increases faster than linearly. $l_{a c}$ is larger than $l_{c}$ at all $\phi_{\text {avg }}>0.826$.

Eq. 4.10 suggests a possible competitive mechanism between the positive correlation and anti-correlation, and define two corresponding length. A microscopic model on particle level is required to understand such a mechanism.

\subsubsection{The lengths crossing Random Loose Packing}

With the help of Prof. Dr. Karen Daniels' group in NCUS, we can have some measurement on the packings around $\phi_{R L P}$. The experiments are conducted on a bi-disperse granular monolayer of photoelastic discs resting on a thin layer of pressurized air. The assembly of particles is comprised of an inner subsystem and a larger bath which differ only in the inter-particle friction coefficient. The inner bath contains 100 discs, while the bath contains 904 discs. The aspect ratio is 1.4, and the two sizes are in equal concentration. Details can be found in Puckett and Daniels, 2012.

Starting from a dilute state, the monolayer is bi-axially compressed by outer walls in a series of short steps. At some global packing fraction $\Phi_{\text {perc }}$, the percolation of force chain is observed and the system jams. Beyond this point, the contact forces grow in strength and the average number of contacts per particle increases. This threshold occurs over a range $0.782<\Phi_{\text {perc }}<0.792$. The random loose packing is defined as the center of the range $\Phi_{R L P} \approx 0.787$.

We measured the correlation between free volumes, $\operatorname{corr}(L)$, in the subsystem, and plot it in Fig. 4.20(a). Due to the size of the subsystem, the measurement only covers the distance till $L=3.7 d_{s}$. The statistics (100 cycles) does no allow us to extract $L_{C}$ from a straight line fit as what has been done for our disc packings. We instead estimate the extension by integrating the area under $\operatorname{corr}(L)$, then divide this area by its maximum at $L=1.5 d_{s}$. The result is shown in Fig. 4.20 (b). It maximum is at $\Phi=0.7811$, just below 
(a)

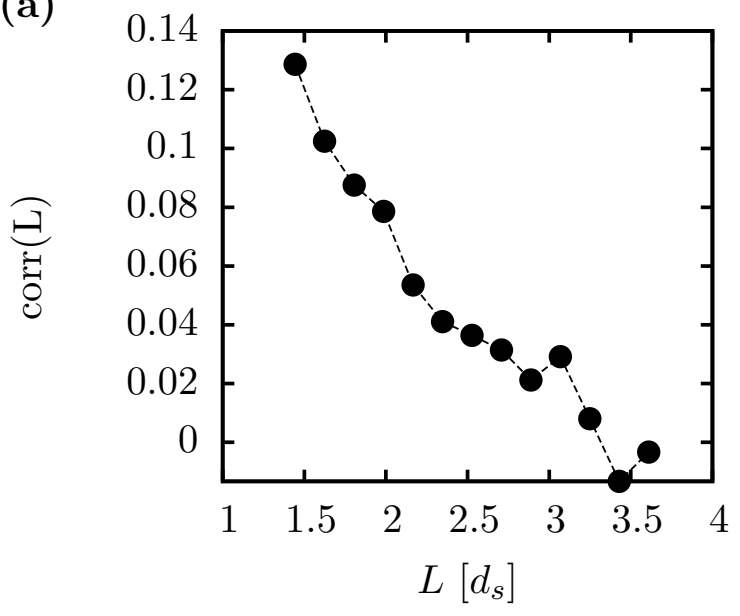

(b)

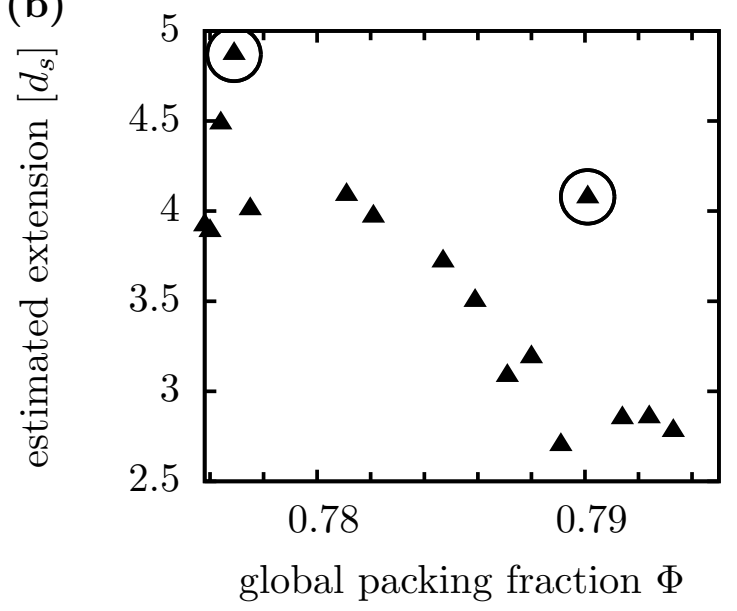

Fig. 4.20: (a): $\operatorname{corr}(L)$ measured in the subsystem for $\Phi=0.788 . d_{s}$ is the diameter of small discs. (b): the extension of $\operatorname{corr}(L)$ is estimated by the area under the curve divided by the height. The global $\Phi_{R L P}$ is around 0.787 . The two points surrounded by the circles are where the packing fraction of the subsystem is larger than that of their right point.

the onset of jammed state. Above this maximum the extension decays till $\Phi=0.7922$, then the extension stays at the same magnitude as $L_{C}$ measured in our packings [cf. Fig. 4.16.

In the dilute unjammed state, the force chains do not percolate the whole system. The local volumes can be rearranged without affecting those beyond the nearest neighbours. Therefore the correlation extension is supposed to decay rapidly beyond the nearest neighbours. In jammed states the force and volume are inter-dependent Blumenfeld et al., 2012]. At the onset of jammed states the system just has a high enough contact number to maintain mechanical stability, and the force chains are sensitive to the local configuration. A local rearrangement might cause the force chains to be changed through the whole system. The changes of the local forces cause the changes of the local volumes in return. Tewari et al. 2009 reported the correlation length of the stress fields in dense granular flow grows as a power law when approaching jamming. In this context correlation of the fluctuation of the local volumes reaches its maximum below random loose packing 6 .

In Fig. 4.20(b) the extension is plotted against the global packing fraction $\Phi$. It should be pointed out that the packing fraction of the subsystem is not monotonic with the global packing fraction $\Phi$. The two triangle data points highlighted by the circles have higher packing fraction than the next data points on the right. The system size is also too small (100 discs) to cover the whole range of correlation, e.g. $\operatorname{corr}(L)$ measured around $\Phi=0.78$ does not decays to zero at even $L \approx 4 d_{s}$, thus the estimated extension is smaller than the actual distance where decorrelation happens. Measurements of correlation of local volumes and the local stresses performed in larger packings could give better insights on the signature of onset of rigidity.

\footnotetext{
${ }^{6}$ This onset is observed as the lower bound of $\Phi_{\text {prec }}$, which is smaller than $\Phi_{R L P}$.
} 


\section{Chapter 5}

\section{Configurational temperature $\chi$ in disc packings}

In Chapter 1 we introduced the statistical mechanics of granular matter suggest by Edwards and Oakeshott [1989], where the Hamiltonian is replaced by a volume function. By analogy to the thermal temperature a configurational temperature, or compactivity, more specific to granular system, $\chi$ is defined coherent to the fluctuation of volume. In this chapter we measure $\chi$ in three different methods and compare them. We will show that the correlation measured in Chapter 4 has consequences on the measurement of $\chi$.

\subsection{Methods}

We first introduce the fundamental definitions and relations of the statistical framework in granular system, then explain three methods of measuring the compactivity $\chi$.

The distribution density is the building block of a statistical framework. The distribution of the volume of a granular system suggested by Edwards and Oakeshott [1989] is:

$$
\begin{aligned}
p_{\chi}(V) & =\int \delta(W(\mathbf{q})-V) p_{\chi}(\mathbf{q}) \mathrm{d} \mathbf{q} \\
& =\mathcal{D}(V) \frac{e^{-V / \chi}}{Z_{\chi}}
\end{aligned}
$$

Where $p_{\chi} \mathrm{q}=\Theta(\mathrm{q}) e^{-W(\mathrm{q}) / \chi}$, and $\mathcal{D}(V)=\int \delta(W(\mathbf{q})-V) \Theta(\mathbf{q})$ dq $\mathbf{q}$ enumerates the number of the mechanical stable states corresponding to a volume $V . Z_{\chi}$ is the partition function:

$$
Z_{\chi}=\int e^{-W(\mathbf{q}) / \chi} \Theta(\mathbf{q}) \mathrm{d} \mathbf{q}=\int e^{-V / \chi} \mathcal{D}(V) \mathrm{d} V
$$

We emphasis that the distribution density in Eq. 5.1 is a hypothesis, analogous to the Boltzmann distribution of energy in thermal system. The whole statistical framework is based on this hypothesis. A possible way to construct such a statistical framework will be discussed later in this chapter.

According to the probability defined in Eq. 5.1, the mean and the variance of the volume are:

$$
\begin{aligned}
\bar{V} & =\int \mathcal{D}(V) \frac{e^{-V / \chi}}{Z_{\chi}} V \mathrm{~d} V=-\frac{1}{Z_{\chi}} \frac{\mathrm{d} Z_{\chi}}{\mathrm{d} \frac{1}{\chi}} \\
\sigma_{\bar{V}}^{2} & =\int \mathcal{D}(V) \frac{e^{-V / \chi}}{Z_{\chi}}(V-\bar{V})^{2} \mathrm{~d} V=\left\langle V^{2}\right\rangle-\bar{V}^{2}
\end{aligned}
$$

Methods have been developed to extract $\chi$ with $p_{\chi}(V), \bar{V}$ and $\sigma_{\bar{V}}^{2}$ measured in experiment and the hypothesised function form in Eq. 5.1. We start with the fluctuationdissipation theorem. 
Fluctuation-dissipation theorem. Analogy fluctuation-dissipation theorem can be established with the above probability density as follows:

$$
\begin{aligned}
\chi^{2} \frac{\mathrm{d} \bar{V}}{\mathrm{~d} \chi} & =-\frac{\mathrm{d} \bar{V}}{\mathrm{~d} \frac{1}{\chi}} \\
& =\frac{1}{Z_{\chi}} \int V^{2} e^{-V / \chi} \mathcal{D}(V) \mathrm{d} V+\int e^{-V / \chi} \mathcal{D}(V) V \mathrm{~d} V \frac{1}{Z_{\chi}^{2}} \frac{\mathrm{d} Z_{\chi}}{\mathrm{d} \frac{1}{\chi}}
\end{aligned}
$$

The first term is the mean of the second moment of $V,\left\langle V^{2}\right\rangle$. Substitution of $\bar{V}$ defined in Eq. 5.3, into the second term, and use of the variance definition in Eq. 5.3 gives:

$$
\chi^{2} \frac{\mathrm{d} \bar{V}}{\mathrm{~d} \chi}=\left\langle V^{2}\right\rangle-\bar{V}^{2}=\sigma_{\bar{V}}^{2}
$$

We now have a relationship analogous to the specific heat and fluctuation of energy in thermal system: $k_{B} T^{2} C_{V}=\sigma_{\bar{E}}^{2}$, where $C_{V}$ is the specific heat at constant volume.

With measured $\sigma_{\bar{V}}^{2}$ integrating Eq. 5.5 can be used to compute the difference of the inverse compacitivity $\frac{1}{\chi}$ :

$$
\left.\Delta \frac{1}{\chi}\right|_{a, b}=\frac{1}{\chi_{a}}-\frac{1}{\chi_{b}}=\int_{V_{b}}^{V_{a}} \frac{\mathrm{d} \bar{V}}{\sigma_{\bar{V}}^{2}}
$$

$\frac{1}{\chi}_{a, b}$ are the inverse compactivity corresponding to state $\bar{V}=V_{a, b}$. Note that Eq. 5.5 only measures the relative change of inverse compactivity. To get the absolute value of $\chi$, a reference point must be introduced [Nowak et al., 1998, Schröter et al., 2005].

The overlapping histogram. Another consequence of the probability density in Eq. 5.1 is that the ratio of two overlapping distributions of volumes must be exponential [Dean and Lefèvre, 2003, McNamara et al., 2009]:

$$
\frac{p_{\chi_{b}}(V)}{p_{\chi_{a}}(V)}=\frac{\mathcal{D}(V) \frac{e^{-V / \chi_{b}}}{Z_{\chi_{b}}}}{\mathcal{D}(V) \frac{e^{-V / \chi_{a}}}{Z_{\chi_{a}}}}=\frac{Z_{\chi_{a}}}{Z_{\chi_{b}}} e^{-\left(\frac{1}{\chi_{b}}-\frac{1}{\chi_{a}}\right) V}
$$

Scale Eq. 5.7 with logarithm on both sides.

$$
\ln \frac{p_{\chi_{b}}(V)}{p_{\chi_{a}}(V)}=\left.V \Delta \frac{1}{\chi}\right|_{a, b}+\ln \frac{Z_{\chi_{a}}}{Z_{\chi_{b}}}
$$

In Eq. 5.8 the slope of $V$ gives $\left.\Delta \frac{1}{\chi}\right|_{a, b}=\frac{1}{\chi_{a}}-\frac{1}{\chi_{b}}$ between two packing fractions. By sweeping the accessible packing fractions in the experiments, the compactivity $\chi$ can be determined by the accumulation of $\Delta \frac{1}{\chi}$ with a constant $\chi_{0}$.

The above two methods rely only on the Boltzmann-like factor $e^{-V / \chi}$ and leave the density of state $\mathcal{D}(V)$ undetermined, thus both have an arbitrary constant to determine the absolute compactivity $\chi$. In the measurement section we are going to determine this constant with the condition that random loose packing is the limit $\chi \rightarrow \infty$. The third method explained below models a complete expression of the probability density $p_{\chi}(V)$ including the density of state $\mathcal{D}(V)$. 
$\Gamma$ distribution model. Aste and Di Matteo 2008] have shown that the distribution of the free volume $V_{f}=V-V_{\min }{ }^{1}$ can be constructed as a $\Gamma$ distribution:

$$
p\left(V_{f}\right)=\left(\frac{k}{\bar{V}_{f}}\right)^{k} \frac{V_{f}^{(k-1)}}{\Gamma(k)} \exp \left(-k \frac{V_{f}}{\bar{V}_{f}}\right)
$$

Where $\bar{V}_{f}$ is the mean free volume, $k$ is the shape factor, and $\Gamma(k)=(k-1)$ ! is the gamma function.

$\Gamma$ distribution has the following relations among the mean free volume $\bar{V}_{f}$, the variance $\sigma_{\bar{V}_{f}}^{2}$ and the shape factor $k$ :

$$
k=\frac{\bar{V}_{f}^{2}}{\sigma_{\bar{V}_{f}}^{2}}
$$

Applying Eq. 5.10 the compactivity $\chi_{\Gamma}$ can computed as:

$$
\chi_{\Gamma}=\frac{\bar{V}_{f}}{k}=\frac{\sigma_{\bar{V}_{f}}^{2}}{\bar{V}_{f}}
$$

This method could determine the compactivity without any arbitrary constant.

\subsection{Measuring the configurational temperature $\chi$}

In this section we are going to apply the three methods previously introduced to measure the configurational temperature $\chi$ for different cluster sizes and packing fractions, then we will compare the results.

To make comparisons of different cluster sizes the experimental data are presented in the Voronoi volume per particle in a cluster $v=V / V_{g}=1 / \phi_{\text {avg }}$ which is the ratio of the total volume $V$ of the Voronoi cells to the total volume of the grains $V_{g}$. As $v$ is defined by $\phi_{\text {avg }}$ it has the unit of averaged particle volume $V_{g} / N$, and so does $\chi$.

\subsection{1 $\chi_{\mathrm{FDT}}$ measured from fluctuation-dissipation theorem}

The application of this method is rather straightforward. The variance $\sigma_{\bar{V}}^{2}$ and the averaged volume $\bar{V}$ are measured for twelve experiments with a given cluster size $N$. A power law, $\sigma_{\bar{V}}^{2}=a\left(V-V_{C r y s}\right)^{b}$ is fitted to the data point [see Fig. 5.1(a)], where $V_{\text {Crys }}$ is the volume of crystalline phase, the hexagonal packing. This power law is suggested for that there is no volume fluctuation in crystalline phase.

With the power law fitting Eq. 5.6 is computed numerically to get $1 / \chi_{\mathrm{FDT}}$ for that $N$ [cf. Fig. 5.1(b)]. We apply the boundary condition $\left.\frac{1}{\chi}\right|_{R L P}=0$ to the integral Eq. 5.6 to get the absolute value of the compactivity $\chi_{\mathrm{FDT}}$ :

$$
\frac{1}{\chi_{\mathrm{FDT}}}=\int_{V}^{V_{R L P}} \frac{\mathrm{d} \bar{V}}{\sigma_{\bar{V}}^{2}}
$$

\footnotetext{
${ }^{1} V_{\min }$ is the minimum attainable volume. In disc packings $V_{\min }=2 \sqrt{3} r_{g}^{2}$ is the volume in hexagonal packing, where $r_{g}$ is the radius of the disk.
} 
(a)

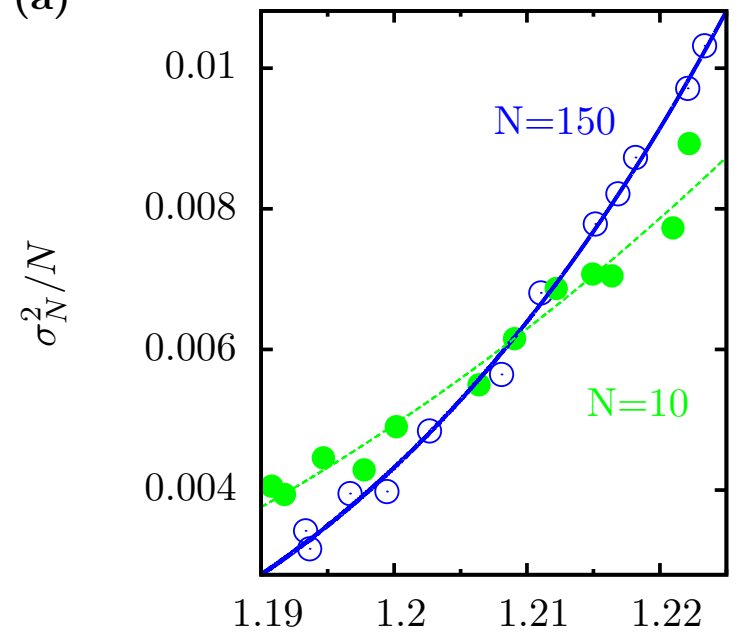

average volume per particle $\bar{v}$ (b)

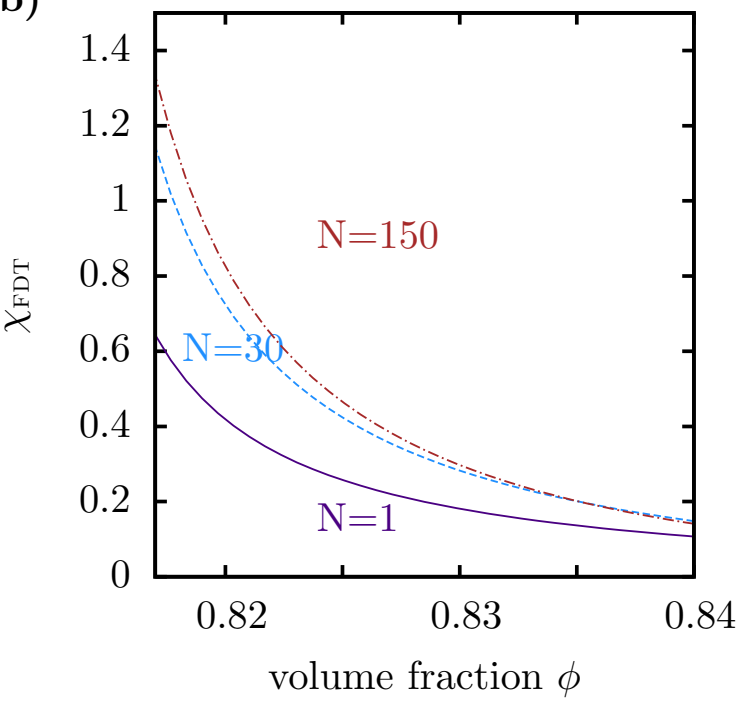

Fig. 5.1: (a): The variance per particle $\sigma_{[N]}^{2} / N$ versus averaged volume per particle $\bar{v}$ of $N=10,150$. The solid curves are the power law fit $\sigma_{\bar{V}}^{2}=a\left(V-V_{C r y s}\right)^{b}$. (b): The compactivity $\chi_{\text {FDT }}$ measured by integrating Eq. 5.6 numerically for $N=1,30,150$.

where $\bar{V}_{R L P}=1 / \phi_{R L P}=1.233$ is averaged over 10 different packings. Those packings are prepared by blowing packings up by a strong air flow then decreasing the flow rate slowly to settle the packing gently.

As can be seen in Fig. 5.1(b) that $\chi_{\text {FDT }}$ is not constant for different cluster sizes $N$ at a given $\phi$. To study the non-intensive compactivity the function of $\chi$ with $N$ is extracted from a vertical line (corresponding to a given $\phi_{\text {avg }}$ ) crossing the curves for different $N$ in

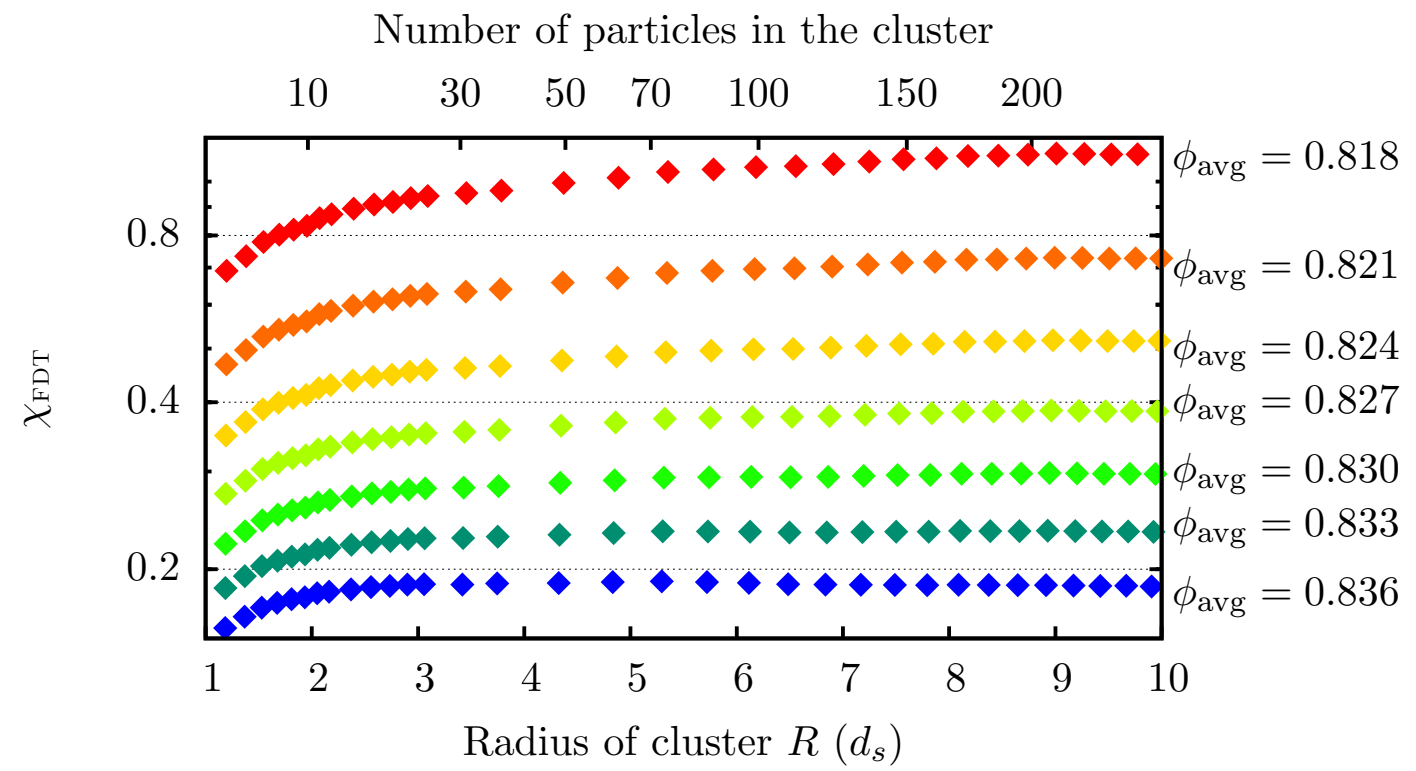

Fig. 5.2: $\chi_{\mathrm{FDT}}$ with cluster sizes at a given $\phi_{\text {avg }}$, which is extracted from the results in Fig. 5.1(b). The cluster size is referred as the radius of the analysis cluster $R \sim \sqrt{N / \phi_{\text {avg }}}$. 
Fig. 5.1(b). The results for different $\phi_{\text {avg }}$ are plotted in Fig. 5.2. In the figure the cluster size is denoted by the radius of cluster $R$ in the unit of diameter of small discs $d_{s}$.

For all packing fractions compactivity $X_{F D T}$ increases with cluster size, which indicates that $X_{F D T}$ is not intensive . Carefully checking the large $N$ development shows that $X_{F D T}$ does not increase monotonically. At relatively high packing fraction e.g., $\phi_{\text {avg }}=0.836$ the compactivity decreases above $R=4 d_{s}$. Nonetheless $X_{F D T}$ of $N>1$ is always larger than that of $N=1$.

\subsection{2 $\chi_{\text {он }}$ measured from overlapping histogram}

In this method we first compute the histograms of volume $V$, then organize the histograms for a given cluster size $N$ in descending order according to their mean volume $\bar{V}$. The ratio of the histograms of neighbouring $\bar{V}$ are computed in their overlapping regions [cf. Fig. 5.3(a)]. The ratio of overlapping histograms for two groups neighbouring packing fractions are shown in Fig. 5.3(b).
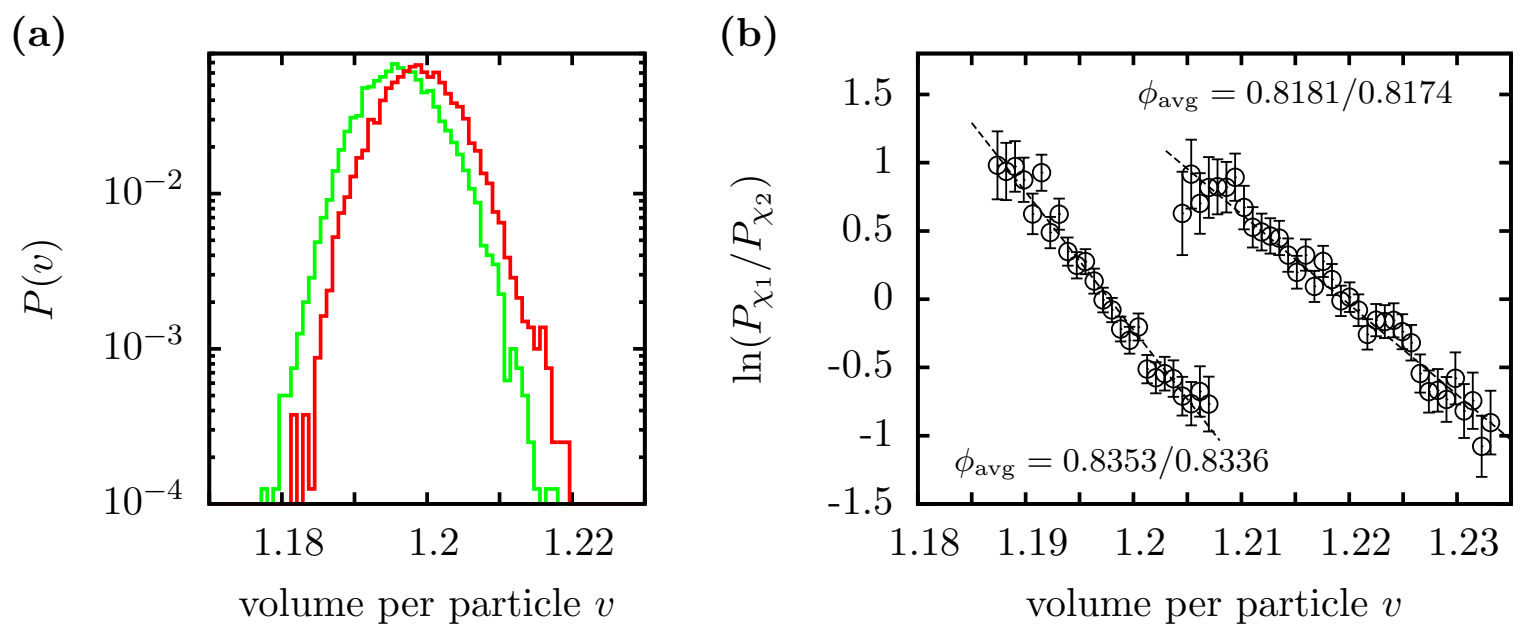

Fig. 5.3: (a): The overlap of distributions at two different packing fractions with $N=150$. (b): Data points are the ratio of two groups of histograms of $N=150$. Dashed lines are the linear fits.

The linearity of the ratio is crucial to this method and to the statistical framework described by equations from Eq. 5.1 to Eq. 5.3. On the other hand, for the large $N$ clusters the histogram appears Gaussian, which predicts that the logarithm of the ratio should be parabolic [McNamara et al., 2009]. Therefore the quality of the linear fit should be checked and compared with the quality of the parabolic fit in the Gaussian case.

The quality of the fit is calculated as its standard deviation $\varsigma^{2}$ :

$$
\varsigma^{2}=\frac{1}{n} \sum_{i=1}^{n} \frac{\left(y\left(x_{i}\right)-y_{i}\right)^{2}}{\Delta y_{i}^{2}}
$$

where $x_{i}, y_{i}$ are the observed volume $v$ and the corresponding ratio $\ln \frac{p_{\chi_{b}}(v)}{p_{\chi_{a}}(v)} \cdot y\left(x_{i}\right)$ is the value of the fit function at $v . \Delta y_{i}^{2}$ is the uncertainty of the measurement which is given 


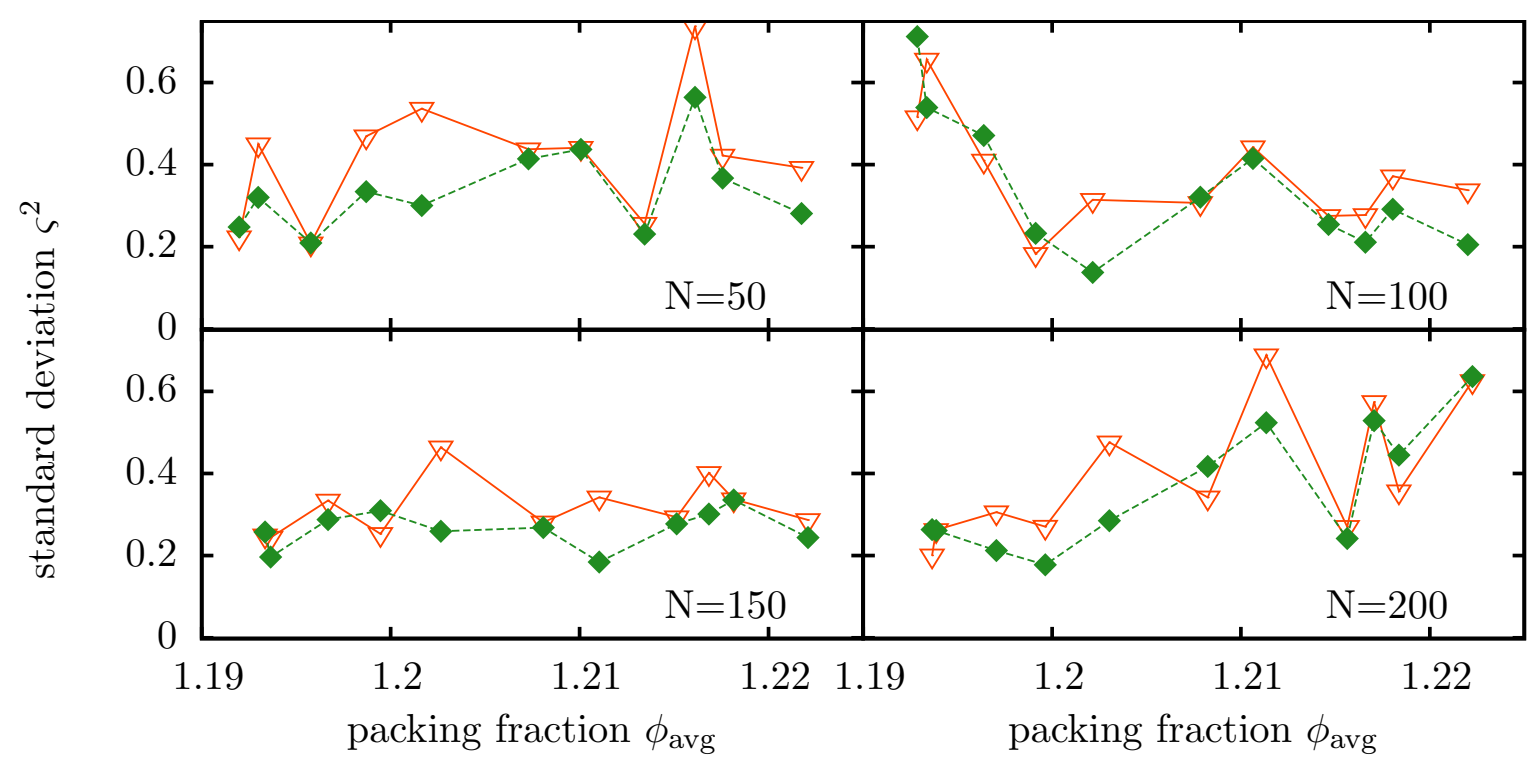

Fig. 5.4: The standard deviation of linear fits (Boltzmann case, green solid square) and parabolic fits (Gaussian case, red open triangle) for $N=50,100,150,200$.

by the uncertainty of the histogram:

$$
\sqrt{\Delta y_{i}^{2}}=\Delta \ln p_{\chi_{b}}-\Delta \ln p_{\chi_{a}} \approx \frac{\Delta p_{\chi_{b}}}{p_{\chi_{b}}}-\frac{\Delta p_{\chi_{a}}}{p_{\chi_{a}}}, \quad\left(\Delta p_{\chi_{a, b}} \ll p_{\chi_{a, b}}\right)
$$

The uncertainty of the histogram, $\Delta p_{\chi}\left(v_{i}\right)=\sqrt{p_{\chi}\left(v_{i}\right) / n_{\text {taps }}}$, is proportional to the square root of the number of independent points in interval $v_{i} \stackrel{2}{ }^{2}$

We calculate $\varsigma^{2}$ of both linear and parabolic fits for the ratio of every pair of neighbouring packing fractions with $N>50$. The results are shown in Fig. 5.4. In all cases $\varsigma^{2}<1$ which indicates the fit is reasonable. Although one quarter of datasets are better fitted by the parabola, on average the standard deviation of linear fit is $13 \%$ better.

This method assumes that the histogram of two packing fraction has the same $\mathcal{D}(V)$, so it can be eliminated by the ratio in Eq. 5.7. Nevertheless this criterion is not sufficient to exclude Boltzmann like distribution in practise. If the ergodicity is not perfectly satisfied in the system, $\mathcal{D}(V)$ for two packing fractions could be different, which makes the ratio non-linear. This might be the possible reason of that the parabolic fit is better for some datasets.

We keep measuring $\chi_{\text {он }}$ with Eq. 5.8. For each dataset a straight line is fitted, and the slope is extracted as the difference of inverse compactivity, $\Delta \frac{1}{\chi_{\mathrm{OH}}}$. Then all $\Delta \frac{1}{\chi_{\mathrm{OH}}}$ are accumulated to get the relative inverse compactivity to the one of the loosest packing, $\chi_{0}$. This $\chi_{0}$ is a constant can not be determined by this method alone we then read the value of $\chi_{\mathrm{FDT}}$ at the loosest packing and make $\chi_{0}=\left.\chi_{\mathrm{FDT}}\right|_{\text {loosest }}$ while keep the relative difference of other data points unchanged. The results are plotted in Fig. 5.5. The two methods are in good agreement [cf. Fig. 5.5].

\footnotetext{
${ }^{2}$ In our experiment, every tap starts with a strong pulse to alternate the packing configuration [cf. Chapter 2. The measured configurations are independent of each other, thus $\Delta p\left(v_{i}\right)=\sqrt{n_{i}} / n_{\text {taps }}$, where $n_{\text {taps }}=8000$ is the total number of taps.
} 


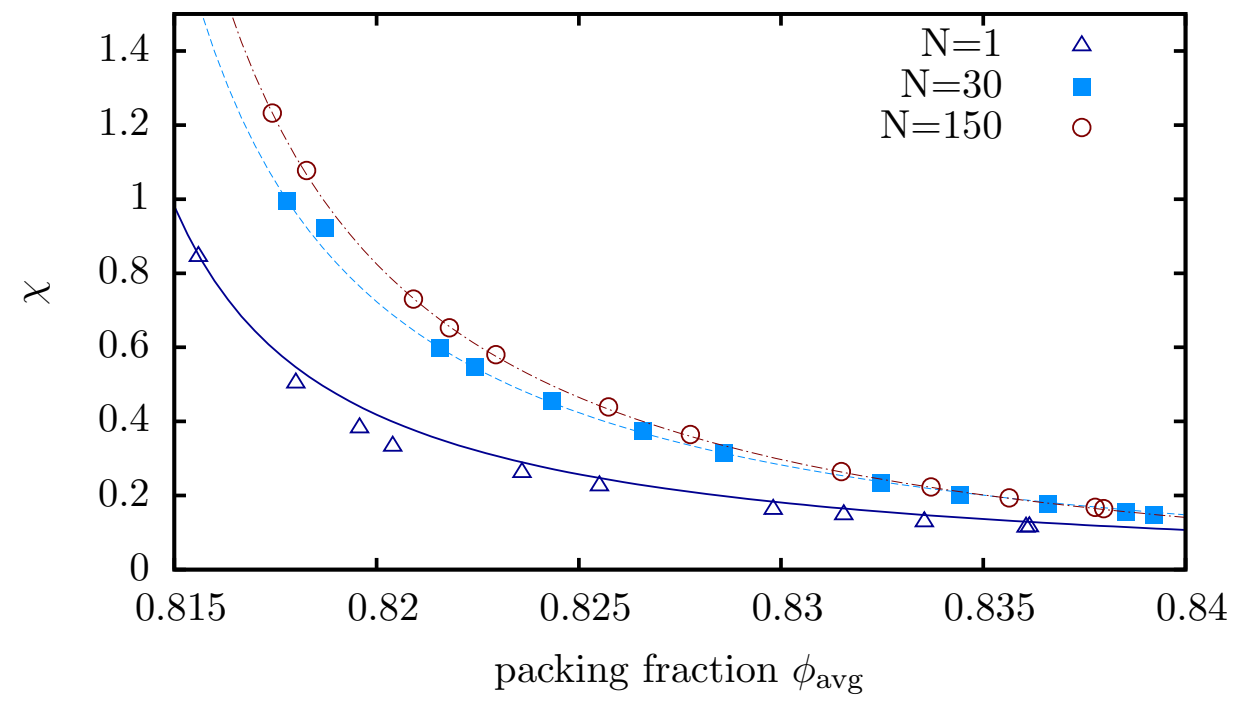

Fig. 5.5: Data points are $\chi_{\text {он }}$ measured by extracting the slope in Eq. 5.8 for $N=1,30,150$. The solid curves are $\chi_{\mathrm{FDT}}$ as in Fig. 5.1.

\subsection{3 $\chi_{\Gamma}$ measured from $\Gamma$ distribution}

We first compute the shape factor $k$ with measured $\operatorname{bar} V_{f}$ and $\sigma_{\bar{V}}^{2}$ by Eq. 5.10 and plot the corresponding $\Gamma$ function together with the measured distributions in Fig. 5.6. The fits look reasonable.

According to some previous work on sphere packings Aste and Di Matteo, 2008, Aste et al. 2007], the shape factor $k$ is robust and equals 12 at different packing fractions and
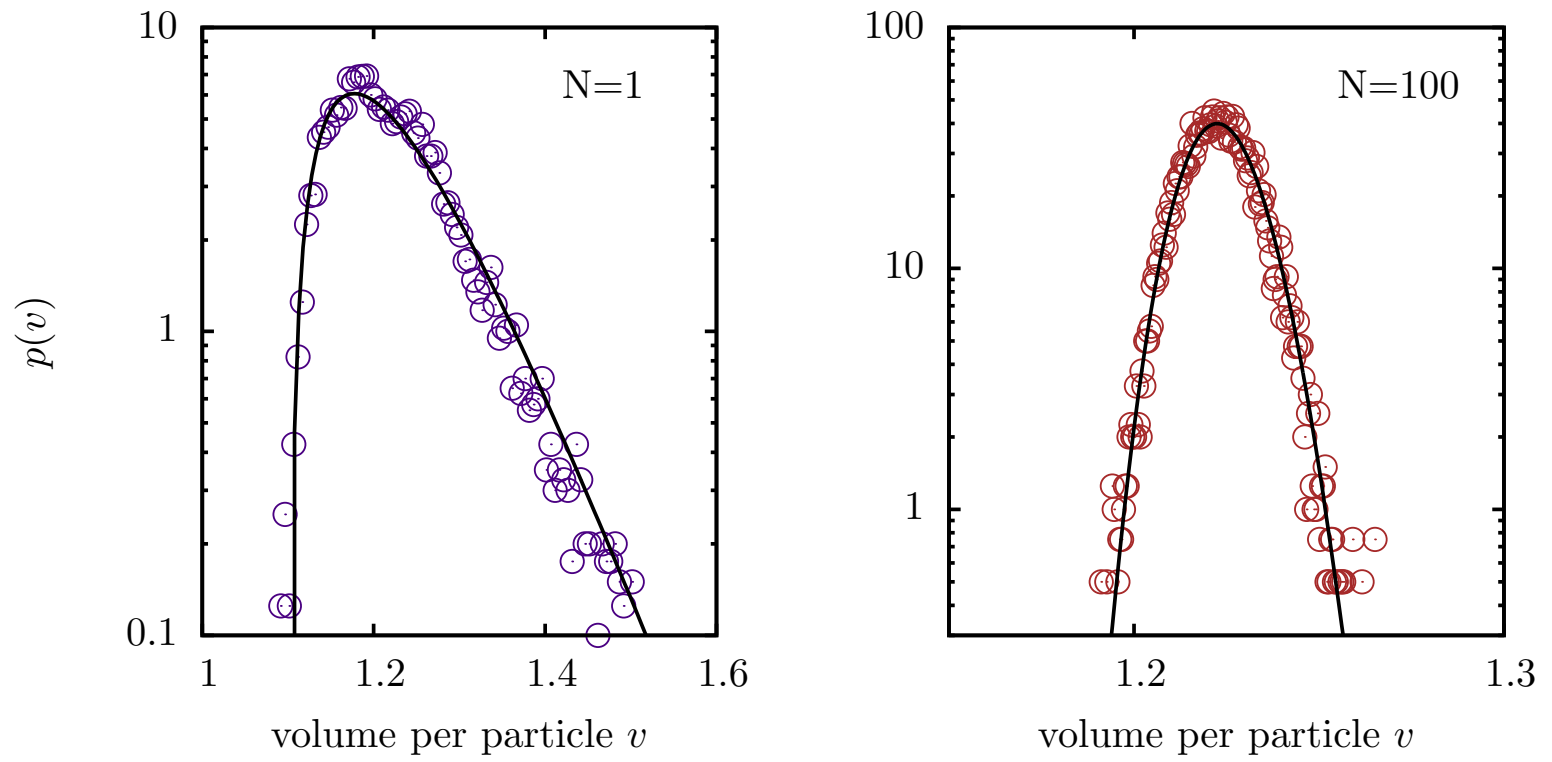

Fig. 5.6: Volume histograms (data points) and the corresponding $\Gamma$ distribution fitting (solid lines) as that in Eq. 5.9 for $\mathrm{N}=1$ (left) and $\mathrm{N}=100$ (right) for $\phi_{\mathrm{avg}}=0.8175$. Note that the value of the histograms has been divided by the binning size to compare with the $\Gamma$ function fitting. 

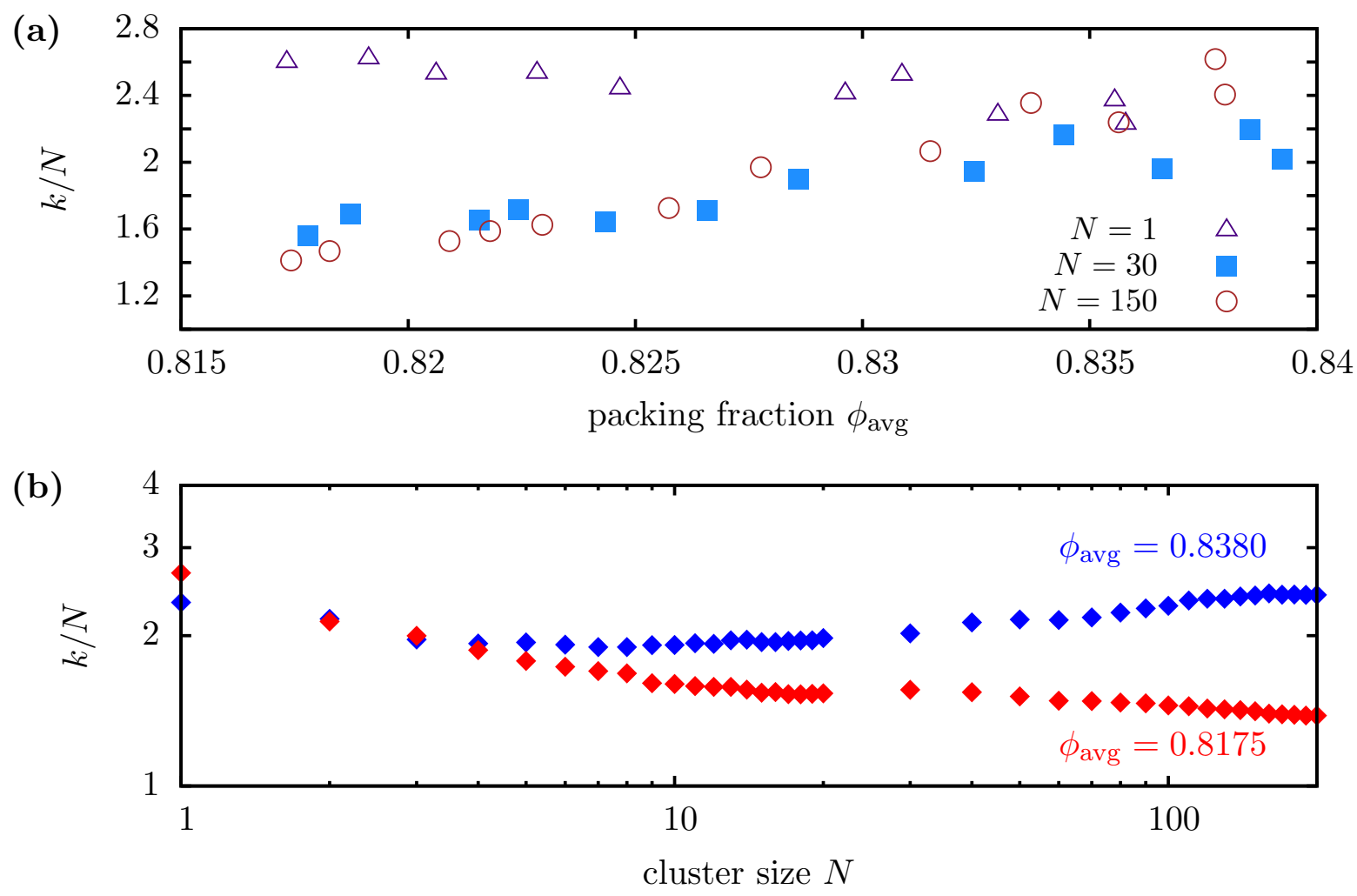

Fig. 5.7: The shape factor $k / N$ versus (a) the packing fraction $\phi_{\text {avg. }}$ for three different cluster sizes, and (b) versus the cluster size $N$ for two different packing fractions.

protocols. Actually the constant $k$ is a consequence of $\Gamma$ distribution model. This can be shown by substituting Eq. 5.11 into the fluctuation-dissipation theorem:

$$
\begin{aligned}
-\mathrm{d} \frac{1}{\chi_{\Gamma}} & =\frac{\mathrm{d} \bar{V}_{f}}{\sigma_{\bar{V}_{f}}^{2}} \\
\sigma_{\bar{V}_{f}}^{2} & =\bar{V}_{f} \chi_{\Gamma}
\end{aligned} \quad \Longrightarrow \mathrm{d} \ln \chi_{\Gamma}=\mathrm{d} \ln \bar{V}_{f}
$$

Integrate Eq. 5.15 one can get $\chi_{\Gamma}=C_{0} \bar{V}_{f}$, where $C_{0}$ is the integral constant. Compare this result with $\chi_{\Gamma}=\bar{V}_{f} / k$ to know that $k=1 / C_{0}$ is a constant. This also indicates that $\chi_{\Gamma}$ is linear with $\bar{V}_{f}$.

To test this prediction we plot the shape factor $k$ of different packing fractions $\phi_{\text {avg }}$ for the cluster size $N=1,30,150$ in Fig. 5.7(a). For $N=1$ the shape factor $k$ stays constant. For $N=30,150 k$ increases linearly with $\phi_{\text {avg }}$, and the slope increases with $N$. $k$ can not be considered to be constant with $\phi_{\text {avg }}$. For example, the value of $k$ at highest $\phi_{\text {avg }}$ is almost $100 \%$ larger than that at lowest $\phi_{\text {avg }}$ for $N=150$.

We also compute the rescaled shape factor $k / N$ for loosest and densest packings at different cluster sizes $N$. The result is shown in Fig. 5.7(b). The rescaled shaper factor $k / N$ decreases with $N<20$. For loose packings $k / N$ keeps decreasing, while for the dense packings $k / N$ increases again and saturates above $N=100$. Those features resemble $\chi_{\text {FDT }}$ versus cluster size $N$ in Fig. 5.2. Recall that Eq. 5.11 expresses $k / N$ which is different from $1 / \chi_{\Gamma}$ by a factor $\phi_{\text {avg }}$, an offset in logarithm scale. Thus the two datasets of $k / N$ 


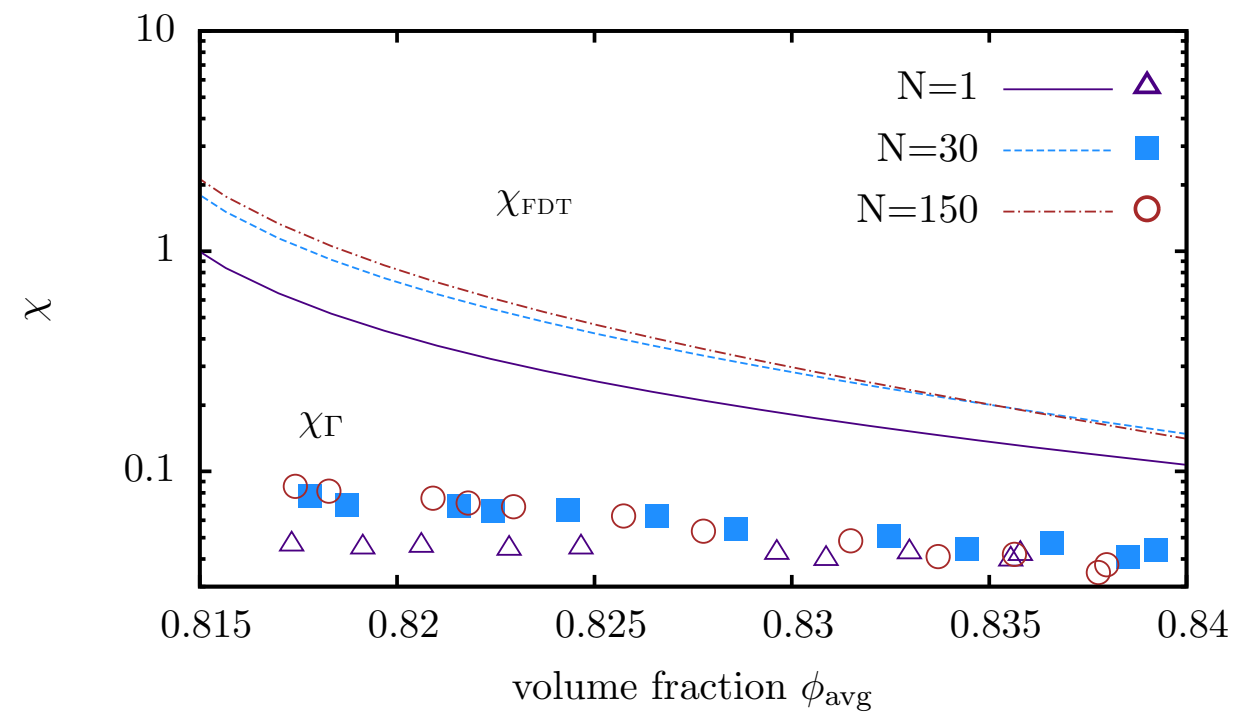

Fig. 5.8: Compactivity $\chi_{\Gamma}$ measured by Eq. 5.11 for three different cluster sizes. $\chi_{\text {FDT }}$ is the same as those in Fig. 5.1(b) and Fig. 5.5.

with $N$ in Fig. 5.7 indicates the same tendency as that of $\chi_{\text {FDT }}$ with $N$ in Fig. 5.2 for loose and dense packings respectively.

Although some inconsistency of the $\Gamma$ distribution model with the experimental data has been found above, we computed the compactivity $\chi_{\Gamma}$ by substituting the measured $\bar{V}_{f}$ and $\sigma_{\bar{V}_{f}}$ into Eq. 5.11. The results are plotted in Fig. 5.8. The magnitude of $\chi_{\Gamma}$ is 10 times smaller than that of $\chi_{\text {он }}$ and $\chi_{\text {FDT }}$. Although quantitatively different the dependence of $\chi_{\Gamma}$ on cluster size $N$ can be observed as well, as expected from the results of $k / N$ in Fig. 5.7(b).

\subsection{Discussion}

The dependence of compactivity $\chi$ on the cluster size $N$ can be understood by fluctuationdissipation theorem together with correlation between Voronoi volumes. The fluctuation of the volume of a cluster including $N$ particles is contributed from both the fluctuation of individual Voronoi cells and the correlation between different cells in the cluster. To write explicitly

$$
\begin{aligned}
\sigma_{[N]}^{2} & =\sum_{k}^{N} \sum_{m}^{N}\left\langle\delta v_{k} \delta v_{m}\right\rangle \\
& =\sum_{k}^{N}\left(\sigma_{k}^{2}+\sum_{m \neq k}\left\langle\delta v_{k} \delta v_{m}\right\rangle\right)>\sum_{k}^{N} \sigma_{k}^{2},
\end{aligned}
$$

where $\sigma_{k}^{2}=\left\langle\delta v_{k}^{2}\right\rangle$ is the fluctuation of a single cell. $\sum_{m \neq k}\left\langle\delta v_{k} \delta v_{m}\right\rangle$ is the correlation between Voronoi volumes. Since the positive correlation domains for all packing fractions, which gives $\sum_{m \neq k}\left\langle\delta v_{k} \delta v_{m}\right\rangle>0$, the variance of the volume of a cluster is larger than the 
sum of that of the single Voronoi cell, i.e. $\sigma_{[N]}^{2}>\sum_{k}^{N} \sigma_{k}^{2}$. Substitute this inequality into Eq. 5.12, one can get $\chi_{N=1}<\chi_{N>1}$.

According to the measurement of the correlation in Chapter 4 the typical extension of positive correlation is around $3 d_{s}$. In Fig. 5.2 the dramatic increasing of $\chi_{\mathrm{FDT}}$ at all $\phi_{\text {avg }}$ happens below the cluster size corresponding to this extension. The development of $X_{F D T}$ above this typical cluster size could be explained by the boundary effect. We will perform a semi-quantitative analysis of the boundary effect later in this chapter. Here we just point out that this effect can be observed in Fig. 5.1(a). At relatively low packing fractions the fluctuation per particle $\sigma_{[N]}^{2} / N$ for $N=150$ cluster is larger than that of $N=10$ cluster. In contrast, at higher packing fractions $\sigma_{[N]}^{2} / N$ for $N=150$ cluster is smaller than that of $N=10$ cluster. The averaged volume at which the fits of the two cluster sizes cross is around 1.21, which is in agreement with the onset of the anticorrelation $\bar{V}_{A C}=1 / 0.8277=1.208$ [cf. Chapter 4]. Equation 5.6 integrates this effect into the measurement of $\chi_{\mathrm{FDT}}$.

In our measurement the result of $\chi_{\Gamma}$ is different from the other two methods [cf. Fig. 5.8. At the first glance this difference may be raised by the application of free volume $V_{f}$ in the $\Gamma$ distribution model rather than the volume $V$ in the other two methods. This is not true. The free volume $V_{f}$ only differs from the volume $V$ by a constant $2 \sqrt{3} / \pi$. This constant shifts the distribution but does not change the shape and the fluctuation, such that $\sigma_{V_{f}}^{2}=\sigma_{\bar{V}}^{2}$ and $\mathrm{d} \bar{V}=\mathrm{d} \bar{V}_{f}$. In the overlapping histogram method, this variable transformation shifts the ratio without changing the slope where $\Delta \frac{1}{\chi_{\mathrm{OH}}}$ is extracted. In the fluctuation-dissipation theorem, replacing $\bar{V}$ with $\bar{V}_{f}$ gives the same power law, $a \bar{V}_{f}^{b}$, and merely shift the integral range in Eq. 5.6. Thus the result of $\chi_{\text {FDT }}$ does not change with the variable transformation as well. So $\chi_{\Gamma}$ is supposed to measure the same physical quantity. The difference of $\chi_{\Gamma}$ measurement makes us to consider the possibility that $\Gamma$ distribution model might not be compatible to the statistical framework described in Eq. 5.1.

Since the Boltzmann distribution $e^{-V / \chi}$ are supported indirectly by the agreement between $\chi_{\mathrm{FDT}}$ and $\chi_{\mathrm{OH}}$, and the free volume and volume represent the same physics, the possible incompatibility of $\Gamma$ distribution model could only be due to the enumeration of mechanically stable states corresponding to a given volume, $\mathcal{D}(V)$ in Eq. 5.1. This can be demonstrated by the range of random packings. As shown in Eq. 5.15, $\chi_{\Gamma}$ is linear with $\bar{V}$ in the model, then the condition $\left.\chi\right|_{R L P}=\infty$ indicates that the random loose packing is at packing fraction $0 . \Gamma$ distribution model apparently includes unjammed states. Nevertheless the distribution Eq. 5.1 is restricted to jammed packings $(\Theta(q) \neq 0)$. This possible flaw of $\Gamma$ distribution model will be discussed with the measurement of $\mathcal{D}(V)$ later in this chapter. In the following we discuss the statistical mechanics for granular system in general.

\subsubsection{Entropy}

Entropy is the crucial quantity characterizing the randomness of a system. The definition of entropy $S$ is:

$$
S=-\int p(x) \ln \frac{p(x)}{\mathcal{D}(x)} \mathrm{d} x=-\int p(x) \ln p(x) \mathrm{d} x+\int p(x) \ln \mathcal{D}(x) \mathrm{d} x,
$$


where $x$ denotes the quantity describing the states in phase space, e.g., $\mathbf{p}, \mathbf{q}$ for a classical system and quantum numbers for a quantum system. $p(x)$ is the probability of observing the system in state $x . \mathcal{D}(x)$ is the density of state, i.e. $\mathcal{D}(x) \mathrm{d} x$ counts the number of microstates within $[x, x+\mathrm{d} x]$. The integral $\int \cdots \mathrm{d} x$ sums over the whole phase space.

The choice of $x$ is subjective as long as the choice can interpret the same physics. For example, in the context of granular packings $x$ could be $\mathbf{q}$ the positions and the orientations (if the particles are not isotropic) of all particles; the volume of the Voronoi cell $V$ which is a function of $\mathbf{q}$; or the equivalent radius of that cell $r=\sqrt[3]{\frac{3}{4 \pi} V} \cdot \mathcal{D}(x)$ which connects different choices is introduced in Eq. 5.17 to assure that the definition of entropy is invariant to parameter transformation Kanatani, 1981. In this work Voronoi volume $V$ is chosen as $x$.

As what has been studied in Chapter 4 the distribution of $V$ becomes sharper as the size of the cluster $N$ increases. This could be seen in Fig. 5.6. In the limit that $N \rightarrow \infty$ and $\bar{V} / N$ is kept constant, where $\bar{V}$ is the average volume, the distribution approaches a delta function $\delta(V-\bar{V})$. $\mathcal{D}(V)$ then determines the asymptotic form of entropy:

$$
\lim _{N \rightarrow \infty} S=\ln \mathcal{D}(\bar{V}) \Delta \bar{V}
$$

$\Delta \bar{V}$ is equal in order of magnitude to the mean fluctuation of $V$ of the system. When the degrees of freedom in the system are independent, $\ln \mathcal{D}(\bar{V}) \Delta \bar{V}$ goes linear with $N$. Equation 5.18 interprets entropy as a measure of the number of states corresponding to the macroscopic volume $\bar{V}$. Then the definition of entropy recovers the form defined by Edwards and Oakeshott 1989].

The essential difference between Eq. 5.17 and Eq. 5.18 is whether the fluctuation of $V$ is negligible in comparison with its mean $\bar{V}$. According to central limit theorem the fluctuation of the distribution increases as $\sqrt{N}$ while its mean increases linearly with $N$. Therefore one should clarify the system size effect while using Eq. 5.18 to compute entropy [McNamara et al., 2009].

Entropy defined by Eq. 5.17 has the same form as that in the Information theory. The information entropy measures uncertainty about information. In the following subsection we are going to discuss the possible application of information entropy on granular statistics.

\subsubsection{Information theory and granular statistics}

Even before Edwards' suggestion, the studies on statistical properties of powder had moved on independently [Kanatani, 1981, Shahinpoor, 1980], and had its root back to Information theory. Jaynes |1957| tried to draw a connection between Information theory and the conventional statistical mechanics. He argued that for a steady system which is characterized by the mean value of a quantity $x$, no matter whether in thermal equilibrium or not, the form of the probability distribution $p(x)$ can be derived by just maximizing the entropy defined by Eq. 5.17 under the conditions such that the observed averaged value is $\bar{x}$, and $p(x)$ is normalized:

$$
\bar{x}=\int x p(x) \mathrm{d} x ; \text { and } \int p(x) \mathrm{d} x=1 .
$$


The resulting distribution would be $p(x)=\mathcal{D}(x) \frac{e^{-x / \lambda}}{Z_{\lambda}}$. The Lagrange multiplier $\lambda$ serves as an intensive parameter characterizing the equilibrium about quantity $x$, which can be determined by the mean value $\bar{x}$. $Z_{\lambda}$ can be determined by the normalization condition. From here all conventional relations, e.g., $\frac{1}{\lambda}=\frac{\partial \ln Z_{\lambda}}{\partial \bar{x}}$, can be constructed. Note that the whole theory is built without the assumptions that are not contained in the laws of mechanics, such as ergodicity, equal a priori probabilities.

In that paper Jaynes [1957] distinguished $p(x)$ into physical and statistical aspects. The former, the state density $\mathcal{D}(x)$, consists of the enumeration of the states of a system and their properties; the latter, $e^{-x / \lambda}$, is the statistical inference. When we talk about a given macroscopic state, we already have some information about it, e.g., $\bar{x}$ by experimental measurement and $\mathcal{D}(x)$ by known physical laws, but we do not have enough information available to lead to certain conclusions about the microscopic states. Entropy in Eq. 5.17 as a concept may be regarded as a measure of this missing information. This is the idea of Shannon's Information Entropy. In this context, the maximum-entropy principle provides the unbiased distribution of microscopic states, which is compatible with the available information. Its immediate consequence, $e^{-x / \lambda}$, does not represents objective knowledge of microscopic states, but rather represents subjective missing of those knowledge. On one hand, if the prediction by applying the resulting distribution is not borne out by the experimental observation, the most reasonable conclusion in this case is that our pre-knowledge represented by $\mathcal{D}(x)$ was not correct, which could give evidence of new laws of physics. An example is the quantum effect on the equipartition of energy of molecular rotation. On the other hand, by having more information about the microstates, entropy can be decreased, thus free energy could be gained. This idea is well known as Maxwell's demon. Toyabe et al. [2010] performed an brilliant information-toenergy conversion experiment. There the position of a dimeric particle is measured and this information is used as a feedback 'driving' the particle upwards a spiral-staircase-like potential.

The above viewpoint has been in debate since it was proposed then, because entropy is an experimentally measurable quantity in thermal equilibrium systems, and its properties were first found empirically. This subject viewpoint of statistics, however, can be generalized to granular packings. We apply this viewpoint to granular packings and summarise the priorly presented results as following. Although the interactions between particles are dissipative, the interaction of Voronoi cells preserve the total volume. The system volume $V$ is used as the characteristic macroscopic quantity, and the configruation of the packing is the microstate. $\mathcal{D}(V)$ enumerates the jammed configurations corresponding to volume $V$. Edwards' distribution $e^{-V / \chi}$ is then the consequence of maximizing entropy in Eq. 5.17. The fluctuation-dissipation theorem and the overlapping histogram rely only on this statistical inference, and the corresponding compactivity, $\chi_{\mathrm{FDT}}$ and $\chi_{\mathrm{OH}}$, agree with each other; $\chi_{\Gamma}$ in the $\Gamma$ distribution model does not agree with the other two methods, because its prediction about $\mathcal{D}(V)$ fails to meet the general physical consideration (we will discuss this in the next section). The global $\chi$ is measured differently from the local $\chi$ because the existence of correlation between microscopic units, Voronoi cells, makes $\ln \mathcal{D}(V)$ non-extensive at small $N$.

In the subjective perspective, rather than $e^{-V / \chi}$, it is the state density $\mathcal{D}(V)$ that represents the relevant physics in granular materials. The studies on $\mathcal{D}(V)$ may integrate the questions about ergodicity, ensembles' usage and equiporability by answering the ques- 
tions like - Is $V$ conserved during microscopic interactions so that it can be used as a proper macroscopic quantity? Which microstates can be explored by the experimental protocol so that those states would be included in $\mathcal{D}(V)$ ? Do different protocols explore the same $\mathcal{D}(V)$ ? More specifically, does the dynamics induced by the experimental protocol put bias on the microstates with same $V$ ? Or are there hidden parameters?

In the last section we will discuss the general properties of $\mathcal{D}(V)$. For now we demonstrate the possible connection between the above questions and the ongoing studies. Voronoi cells tessellate the volume completely, but quadrons do not when 'rattlers' present Ciamarra, 2007]. The rattlers do not support other particles mechanically, such that they do not affect the stress state and force network in static piles, thus might be considered irrelevant to the physics of granular materials without body forces. Consequently the stress state is used to characterize static granular systems, besides the volume, where the Angoricity-compacitivity ensemble [Blumenfeld and Edwards, 2009, Blumenfeld et al., 2012] and the stress ensemble Henkes and Chakraborty, 2009, Henkes et al., 2007, Lois et al., 2009] come into play.

\subsection{Further results}

In the previous sections we have seen that the compactivity $\chi$ is a function of $N$ due to the correlation contribution to the fluctuation of volume [cf. Fig. 5.1(a) and Fig. 5.2. This effect is studied first in this section. Then the density of state $\mathcal{D}(V)=\int \delta(W(\mathbf{q})-$ $V) \Theta(\mathbf{q})$ dq $[$ cf. Eq. 5.1] for individual particles in the packing is extracted and the general properties of $\mathcal{D}(V)$ is discussed.

\subsubsection{The finite size effect}

Fig. 5.1(a) indicates the correlation contribution to the fluctuation of volume. The dependence of $\chi$ on $N$, in all methods, can be understood by this contribution. In Chapter 4 we showed that the fluctuation of the volume of a cluster, $\sigma_{N}^{2}$, is composed of the fluctuation of individual particles and the contribution of the correlation, i.e. $\sigma_{N}^{2}=N \sigma_{0}^{2}+\sum_{i} C_{i}$, where $C_{i}=\sum_{j \neq i}\left\langle\delta v_{i} \delta v_{j}\right\rangle$ is the correlation between local volume $i$ and all other local volumes [cf. Eq. 4.2 and Eq. 5.16. In the following we are going to perform a semiquantitative analysis of the development of the variance of volume, $\sigma_{N}^{2}$, with cluster size $N$.

Consider the central particle of a cluster with a radius $R>L_{C}$ and a density $\varrho$. The correlation between the considered particle and all others, $C$, is:

$$
C=\varrho \int^{L_{C}} \operatorname{corr}(L) 2 \pi L \mathrm{~d} L
$$

$L$ is the distance from the considered particle. $\varrho 2 \pi L \mathrm{~d} L$ enumerates the number of particle in the spherical shell from $L$ to $L+\mathrm{d} L$ surrounding the considered particle. $\operatorname{corr}(L)$ is the two-point volume correlation which has been measured in Chapter 4 . The integral covers the range from the nearest neighbour of the considered particle to the extension of the correlation $L_{C}$. 
Recall that in loose packing $\operatorname{corr}(L)$ decays from a maximum value $C_{0}$ to zero [cf. Fig. 4.12]. Because the particles are discrete in the system, we compute the integral in Eq. 5.19 in two regions of $L$ separately, i.e. $\int \ldots \mathrm{d} L=\left(\int^{d_{g}}+\int_{d_{g}}\right) \mathrm{d} L . d_{g}$ denotes the averaged distance between nearest neighbours. In the region where $L<d_{g}$ particles are neighbours of the considered particle, and each contributes $C_{0}$ to the integral in Eq. 5.19. The number of particles in this region is $\varrho \pi d_{g}^{2}-1$. The -1 subtracts the considered particle. In the region where $L>d_{g}$ the system is treated continuously. Without losing generality we propose $\operatorname{corr}(L)=\frac{C_{0}}{d_{g}-L_{C}}\left(L-L_{C}\right)$ in this region. This form decreases linearly with $L$ and satisfies that $\operatorname{corr}\left(d_{g}\right)=C_{0}$ and $\operatorname{corr}\left(L_{C}\right)=0$. Substitute the contributions of these two regions into Eq. 5.19 .

$$
\begin{aligned}
C & =\varrho\left(\int^{d_{g}}+\int_{d_{g}}^{L_{C}}\right) \operatorname{corr}(L) 2 \pi L \mathrm{~d} L \\
& =C_{0}\left(\varrho \pi d_{g}^{2}-1\right)+2 \pi \varrho \int_{d_{g}}^{L_{C}} \frac{C_{0}}{d_{g}-L_{C}}\left(L-L_{C}\right) L \mathrm{~d} L \\
& =C_{0}\left(\varrho \pi d_{g}^{2}-1\right)+2 \pi \varrho \frac{C_{0}}{d_{g}-L_{C}}\left(-\frac{L_{C}^{3}}{6}+\frac{L_{C} d_{g}^{2}}{2}-\frac{d_{g}^{3}}{3}\right)
\end{aligned}
$$

$\varrho, C_{0}$ and $L_{C}$ has been measured in experiments. Therefore Eq. 5.20 can be used to compute the 'complete' correlation contribution of a particle. The term 'complete' indicates that the integral covers the whole extension of $\operatorname{corr}(L)$. If not all correlated neighbours are included in the cluster, the resulting correlation contribution is 'incomplete' and smaller than ${ }^{3} C$ computed by Eq. 5.20 .

Now consider the whole cluster. According to whether the correlation contribution of particles is complete, we calculate the variance of the cluster volume, $\sigma_{N}^{2}$, by summing two portions. One portion consists of the particles at least $L_{C}$ from the boundary (the white area in the inset of Fig. 5.9p. Those particles have all its correlated neighbours included in the cluster, thus its contribution to $\sigma_{N}^{2}$ is the sum of the variance of individual particles, $\sigma_{0}^{2}$ and the correlation $C$ computed in Eq. 5.20 . The number of particles of this portion is $\varrho \pi\left(R-L_{C}\right)^{2}$. The other portion consists of the particles in the shaded shell in the inset of Fig. 5.9. Those particles do not have all its correlated neighbours inside the cluster, thus have a correlation contribution different from $C$. We denote the averaged correlation contribution of these particles as $C^{\prime}$. The number of particles inside the shaded area is $\varrho \pi\left(2 R L_{C}-L_{C}^{2}\right)$. Now $\sigma_{N}^{2}$ can be expressed as the sum of the two portions:

$$
\begin{aligned}
\sigma_{N}^{2} & =\varrho \pi\left(R-L_{C}\right)^{2}\left(\sigma_{0}^{2}+C\right)+\varrho \pi\left(2 R L_{C}-L_{C}^{2}\right)\left(\sigma_{0}^{2}+C^{\prime}\right) \\
& =N \sigma_{0}^{2}\left[1+\frac{C}{\sigma_{0}^{2}}+\frac{L_{C}^{2}-2 R L_{C}}{R^{2}} \frac{C-C^{\prime}}{\sigma_{0}^{2}}\right] \\
& =N \sigma_{0}^{2}\left[1+\varepsilon+\frac{L_{C}^{2}-2 R L_{C}}{R^{2}} \frac{\varepsilon}{\eta}\right]
\end{aligned}
$$

where the condition of the total number of particle in the cluster $N=\varrho \pi R^{2}$ is implemented, and $\varepsilon=C / \sigma_{0}^{2}$ and $\eta=\frac{C-C^{\prime}}{C}$. The averaged correlation contribution in the shaded

\footnotetext{
${ }^{3}$ Strictly speaking, the incomplete correlation could be larger than the complete one, when anticorrelation happens. This case will be discussed later in this subsection.
} 


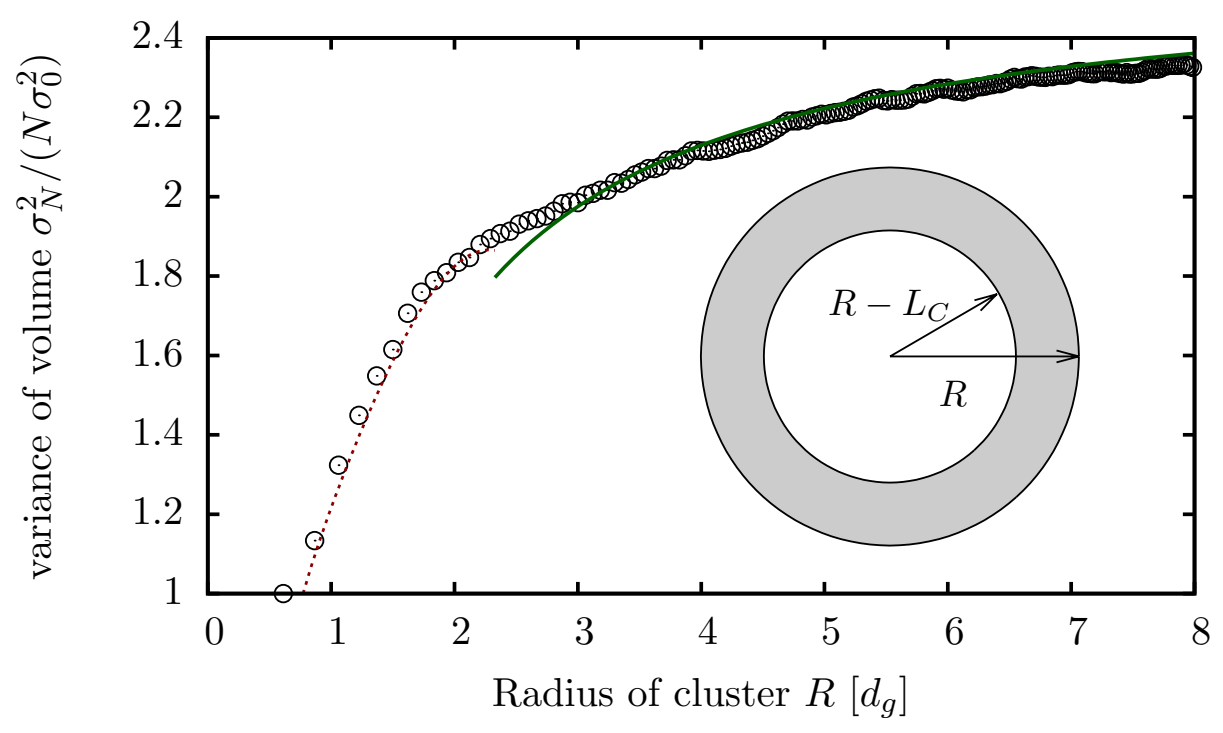

Fig. 5.9: MAIN PLOT: The variance of the volume of the cluster $\sigma_{N}^{2}$ versus the cluster radius $R$. The number of particles inside the cluster is $N=\varrho \pi R^{2}$, where $\varrho$ is the density. The variance $\sigma_{N}^{2}$ is measured from $N=1$ to $N=200$ for packing fraction $\phi_{\text {avg }}=0.8231$ and is rescaled by the variance of $N=1, \sigma_{0}^{2}$. The solid green curve and the dash red curve are computed by Eq. 5.21 and Eq. 5.22 without fitting parameters. Details can be found in the text. INSET: A sketch of a circular cluster with $R$. The cluster are decomposed into two portions. One is the white area within $R-L_{C}$. The particles in this area has all its correlated neighbours inside the cluster. The other is the shaded area. The particles in this area have only partially of its correlated neighbours inside the cluster.

shell, $C^{\prime}$, depends on the cluster size $R$, so does $\eta$. We use $\eta=4-\frac{2 L_{C}}{R}$, so that $R \rightarrow \infty$, $\eta \rightarrow 4$ and $R \rightarrow L_{C}, \eta \rightarrow 2$.

On the other hand, in a cluster with radius $R<L_{C}$, the integral in Eq. 5.19 only partially cover the correlation extension, such that the correlation contribution of the central particle is a function of the cluster size, $C(R)=2 \pi \varrho \int^{R} \operatorname{corr}(L) L \mathrm{~d} L$. The other $N-1$ particles in the cluster approximately have a correlation contribution half of $C(R)$. Thus the variance of the volume in this case is:

$$
\begin{aligned}
\sigma_{N}^{2} & =N \sigma_{0}^{2}+C(R)+(N-1) \frac{C(R)}{2} \\
& =N \sigma_{0}^{2}\left(1+\frac{N+1}{2 N} \frac{C(R)}{\sigma_{0}^{2}}\right)
\end{aligned}
$$

Where $C(R)$ can be computed by replacing the upper bound in Eq. 5.20 with $R$.

All quantities in Eq. 5.21 and Eq. 5.22 are measurable. We extract $C_{0}=0.23 \sigma_{0}^{2}$ and $L_{C}=2.87 d_{s}$ from the experimental data $\operatorname{corr}(L)$ for $\phi_{\text {avg }}=0.8231$ and substitute them into Eq. 5.21 and Eq. 5.22, and plot the results together with the experimentally measured $\sigma_{N}^{2}$ in Fig. 5.9. The models capture the tendency at large and small $R$, but fails in the intermediate range $L \in\left[L_{C}, L_{C}+d_{g}\right]$. In this range the model (Eq. 5.21) considers that the number of particles that has the complete contribution $C$ is less than 1 , thus underestimates the contribution of the central particle. 
Above we only consider the case of positive correlation where the integral of Eq. 5.19 increases monotonically with its upper bound. Nevertheless with the appearance of the anti-correlation the integral decreases again when the upper bound is beyond $L_{C}$ and the integral enters the anti-correlation regime. The resulting complete correlation $C$ is smaller than the incomplete correlation $C\left(L_{C}\right)$, which makes $\sigma_{N}^{2} / N$ first increase with $N$ then decrease above a certain cluster size. This cluster size corresponds to where $\operatorname{corr}(L)$ becomes negative. This effect can be observed in Fig. 5.1(a) where for $\phi_{\text {avg }}$ above the onset of anti-correlation, $\phi_{A C}, \sigma_{N}^{2} /\left.N\right|_{N=10}>\sigma_{N}^{2} /\left.N\right|_{N=150}$, while below $\phi_{A C}$ it is opposite. The large $N$ behaviour with anti-correlation can be obtained using the same procedure as in Eq. 5.21 but with a negative $\eta$.

Recall that the fluctuation dissipation theorem (Eq. 5.6) compute $\chi$ by integrating $1 / \sigma_{N}^{2}$. Substitute Eq. 5.21 into Eq. 5.6 .

$$
\frac{1}{\chi}=\int_{V_{R L P}} \frac{\mathrm{d} \bar{V}}{\sigma_{N}^{2}}=\int_{V_{R L P}} \frac{\mathrm{d} \bar{V}}{N \sigma_{0}^{2}\left(1+\varepsilon+\mathcal{O}\left(\frac{1}{R}\right)\right)}
$$

where $\mathcal{O}\left(\frac{1}{R}\right)=\frac{L_{C}^{2}-2 R L_{C}}{R^{2}} \frac{\varepsilon}{\eta}$. In general $\sigma_{0}^{2}$ and $\varepsilon$ are a function of $\bar{V}$ but constant with the cluster radius $R$. When the cluster radius $R$ is much larger than the correlation extension, $\chi$ behaves qualitatively as $1+\varepsilon+\mathcal{O}\left(\frac{1}{R}\right)$ which resembles the development of $\sigma_{N}^{2}$ at large $R$. This explains the observation in Fig. 5.2 .

\subsubsection{Density of state}

Notice: In this section the result of $\mathcal{D}(V)$ is shown for local volume $N=1$, and the discussions about random loose packing and random close packing is only quantitative and highly speculating.

For a jammed packing the density of state $\mathcal{D}(V)=\int \delta(W(\mathbf{q})-V) \Theta(\mathbf{q})$ dq enumerates the number of jammed configurations corresponding to the system volume $V$. The volume function $V=W(\mathbf{q})$ and the mechanical stability $\Theta(\mathbf{q})$ represent the geometrical and mechanical constraints, respectively. They interplay in the formation of jammed states. The empirical range of random jammed states is bounded by Random Loose Packing (RLP) [Scott, 1962] and Random Close Packing (RCP) [Bernal and Mason, 1960]. Although those bounds are well known, they still lack precise definition due to the unclear relevant physics. For instance, Onoda and Liniger [1990] implied that RLP is the loosest random packing that is mechanically stable. More recently Ciamarra and Coniglio 2008] showed that there are actually many mechanically stable states below the experimentally observed RLP. On the other hand, RCP is suggested to be the state with $\ln \mathcal{D}(V)=0$ in the same work and in Briscoe et al. 2008]. It is interesting to ask how the entropy develops above RCP to the more ordered crystalline phase. In contrast, Torquato et al. [2000] argued that RCP should be replaced by Maximally Random Jammed state. The bond-orientational order was proposed to identify the maximal randomness packing fraction, $\phi_{M R J} \approx 0.64$ for mono-sphere packing. Other work will be discussed with our results about $\mathcal{D}(V)$.

Without getting hands on the issue of the definitions yet, we term the loosest mechanically stable packing as $\phi_{J}$ and the corresponding volume as $V_{J}=1 / \phi_{J}$, and we term the 


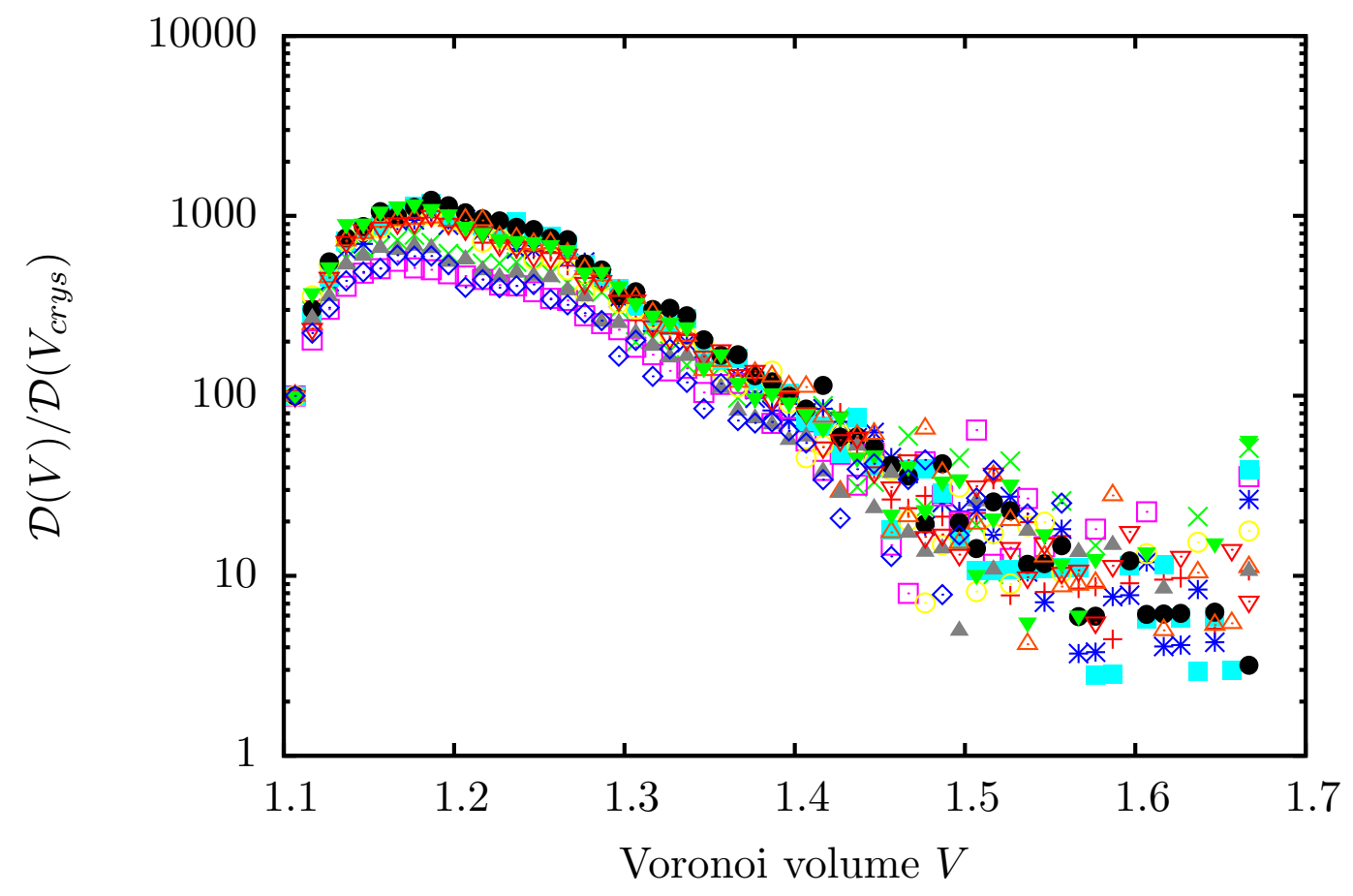

Fig. 5.10: The state density function $\mathcal{D}(V)$ of $N=1$ for all twelve experiments. In the plot $\mathcal{D}(V)$ is rescaled by its value at crystalline volume $V_{\text {crys }}$. Binning $\Delta V=0.01$.

volume of the crystalline phase as $V_{C r y s}$. Above $V_{J}$, no mechanically stable states can be constructed, such that $\mathcal{D}\left(V>V_{J}\right)=0$, whereas at the crystalline phase no free volume is left in the system.

Now consider how $\mathcal{D}(V)$ develops between $V_{\text {Crys }}$ and $V_{J}$. For a state close to $V_{J}$ large free space is supposed to lower the average contact number which is against mechanical stability $\Theta(\mathbf{q}) \neq 0$. In this sense, when approaching $V_{J}$ from below, $\mathcal{D}(V)$ is expected to decrease with $V$ and vanish when $V>V_{J}$. On the other hand, for a state far above $V_{J}$ the small $V$ leaves too little free space to construct many different configurations? An extreme case is the crystalline volume $V_{C r y s}$ which has only one corresponding state in monosize disc packings, the hexagonal packing. Therefore $\mathcal{D}(V)$ increases with $V$ in the regime close to $V_{\text {Crys }}$. By considering the above extreme cases $\mathcal{D}(V)$ is not monotonic with $V$ but has a maximum between $V_{C r y s}$ and $V_{J}$.

To test the above conclusion, we compute $\mathcal{D}(V)$ with the probability density in Eq. 5.1 and the measured $\chi$ by:

$$
\frac{\mathcal{D}(V)}{Z_{\chi}}=p_{\chi}(V) e^{\frac{V}{\chi}} ;\left(\chi=\chi_{\mathrm{oH}}\right)
$$

The partition function $Z_{\chi}$ is an integral over the whole phase space, hence is independent of $V$. Then $\frac{\mathcal{D}(V)}{Z_{\chi}}$ is rescaled by its value at the crystalline volume $V_{\text {crys }}=1.10$ to eliminate $Z_{\chi}$, because $\mathcal{D}\left(V_{\text {crys }}\right)$ is supposed to be 1 . The result for $N=1$ is plotted in Fig. 5.10 for all twelve experiments. The computed $\mathcal{D}(V)$ is indeed concave. Any theoretical model should predict such a concave $\mathcal{D}(V)$, yet the density of state used in $\Gamma$ distribution

\footnotetext{
${ }^{4}$ hard-core and constant pressure.
} 
(Eq. 5.9) increases with $V$ monotonically. In fact the $\Gamma$ distribution model does not include mechanical stability condition [Aste et al., 2007]. This might be the reason why $\chi_{\Gamma}$ fails to match the other two methods.

Additionally the state density in Fig. 5.10 collapse for different $\bar{V}$. The statistical framework expects that $\mathcal{D}(V)$ does not depend on the protocol and compactivity $\chi$, but can be determined alone by the properties of particles and the geometrical and mechanical constraint, such as the friction coefficient, the sizes and the shapes of particles, the volume $V$ and the boundary stress. Recall that we introduce two tapping protocols in Chapter 2 . Results in Fig. 5.10 indicates that the two protocols explore the same $\mathcal{D}(V)$.

With $\mathcal{D}(V)$ we can discuss Random Loose Packing (RLP) and Random Close Packing $(\mathrm{RCP})$ in more details. RLP in the statistical framework is defined as the limit $\chi \rightarrow$ $\infty$, such that $e^{-V / \chi}=1$ is constant with $V$, i.e. all microstates with different $V$ are equilprobable. So defined volume per particle at RLP,

$$
V_{R L P}=\frac{\int \mathcal{D}(V) V \mathrm{~d} V}{\int \mathcal{D}(V) \mathrm{d} V},
$$

is computed as $1.2284 \pm 0.0033$ which corresponds to a packing fraction $\phi_{R L P}=0.8140 \pm$ $0.00225^{5}$ Note that this definition of $\phi_{R L P}$ is averaged over the whole phase space, not a certain point or region in the phase space. There must be states below $\phi_{R L P}$ which are still possible in principle but are very unlikely. When the system size $N$ increases, $\ln \mathcal{D}(V)$ increases extensively, thus the chance to realize those states globally is even smaller. That is the reason why the global packing fraction below $\phi_{R L P}$ has never be observed experimentally, but only be reported in the simulation by carefully chosen initial state and gentle deposition protocols [Ciamarra and Coniglio, 2008].

A candidate statistical definition of RCP stems from the observation that above RCP some crystal order is unavoidable. This is similar to the liquid-solid transition. A phase transition between the random state and the crystal state must be first-order. Following this routine, it is suggested that the entropy decreases monotonically with density but has a discontinuity at RCP [Jin and Makse, 2010, Radin, 2008. Above this point random clusters and crystal clusters co-exist in the system, and the entropy decreases in a different manner with density. This discontinuity of entropy might be raised in $\mathcal{D}(V)$. With $V=V_{\text {Crys }}+\delta V$ the local packing suddenly becomes random and state density $\mathcal{D}(V)$ is supposed to have a abrupt increase. We can not confirm this increase in Fig. 5.10 due to the uniform finite binning size $\Delta V=0.01$.

$\mathcal{D}(V)$ displayed in Fig. 5.10 has a maximum at $V_{M}=1.1783 \pm 0.0083$ corresponding to a packing fraction $0.8487 \pm 0.0060$. This packing fraction characterizes the largest number of configurations or microstates. Note that $\mathcal{D}(V)$ in Fig. 5.10 is for $N=1$. When we consider the global system this maximum gains more weight $(\ln \mathcal{D}(V)$ increases extensively at large $N$ limit). $V_{M}$ is different from MRJ suggested in Torquato et al., 2000] which characterizes the maximum spatial random states.

It should be emphasised that the quantitative features of $\mathcal{D}(V)$ must depend on the external stresses. Besides, the value of $V_{R L P}$ and $V_{M}$ are extracted from the local $\mathcal{D}(V)$ $(N=1)$. Globally those values will be shifted due to the correlation between local volumes. The measurement of $\mathcal{D}(V)$ is performed with the condition that $\mathcal{D}\left(V_{\text {Crys }}\right)=1$

\footnotetext{
${ }^{5} V_{R L P}$ is computed over the twelve experiments, so is $V_{M}$ below.
} 
to cancel the partition function $Z_{\chi}$. When preparing random packings we avoid the appearance of crystalline nucleations, so for $N>1$ the statistics near $V_{C r y s}$ is very low. Thus for large $N$ we can not measure $\mathcal{D}(V)$ as for $N=1$. Nevertheless we can extract $V_{M}$ and $V_{R L P}$ out of the computed $\mathcal{D}(V) / Z_{\chi}$ in Eq. 5.24 for $N>1$. For instance, $\left.V_{M}\right|_{N=200}=1.229$ and $\left.V_{R L P}\right|_{N=200}=1.230$ for $N=200$. Those two values are close to the global RLP that we measured in the experiments, $V_{R L P}=1.233$. Since the number of configurations corresponding to $V_{M}$ dominates over other $V$, it is expected that in sufficient large cluster $V_{R L P}$ measured in Eq. 5.25 by averaging $\mathcal{D}(V)$ is identical to $V_{M}$.

Although in our experiments $\mathcal{D}(V)$ is found to be independent of tapping protocols, the properties of granular matters might depend on protocols in general. An external perturbation is always needed to move the granular system from one mechanically stable configuration to another. Many protocols stimulate the whole system rather than individual particles. Consequently, even history independent protocols may have different sensitivities to energy landscape in the configuration space leading. One example is that the stress distribution beneath a sand pile depends on the chosen protocol and preparation history [Vanel et al., 1999].

Gao et al. 2009] performed a direct measurement of the mechanically stable configuration space of 7 frictionless discs in a two dimensional box generated by tapping or quasi-static compression. There it was found that the fraction of configurations sampled by quasi-static compression decreases with increasing strain, which indicates non-ergodic evolution; besides, individual configurations for a given system geometry are not equiprobable, however are relatively insensitive to preparation.

As discussed in the previous section, from the perspective of the information theory one can still define the intensive parameter $\chi$ in experiments, in despite of those complex issues about the phase space, e.g. the protocol dependence of the exploration of the phase space and the non-equilprobability of microstates. It has also been shown that equiprobability is unnecessary for the existence of intensive parameters [Bertin et al., 2007], and the protocol-dependent degeneracy function, if exists, can be included in $\mathcal{D}(V)$ [Henkes and Chakraborty, 2009, Lechenault et al., 2006. 


\section{Conclusion of Part I}

In Chapter 4 we presented the measurement of the two-point correlation between local volumes for binary disc packings. A short-ranged positive correlation over the whole range of packing fractions $0.8175-0.8380$. Above a volume fraction of $0.8277 \pm 0.0005$ we observe anti-correlation in the free Voronoi volumes. These anti-correlations reach a maximum at a distance of about 3.5 small particle diameters. They then decay exponentially with distance, with an exponent growing linearly with packing fraction. The positive correlation is confirmed about Random Loose Packing in another experimental protocol. Combining the results on two experimental protocols the extension of the positive correlation is expected to decreases with packing fraction, and has its maximum near Random Loose Packing.

In Chapter 5 we followed the statistical framework suggested by Edwards and Oakeshott [1989] to define the configurational temperature, or compactivity $\chi$, in binary disc packings. We apply three methods to measure $\chi$ versus packing fraction for different cluster sizes: the fluctuation-dissipation theorem, the overlapping histogram and $\Gamma$ distribution model. The results of the first two methods agree with each other, whereas $\Gamma$ distribution model does not agree with the other two methods. In all three methods $\chi$ at a given packing fraction depends on the cluster size. This dependence is due to the correlation between local volumes, and is analysed quantitatively. With the measured $\chi$ one can extract the density of state $\mathcal{D}(V)$ from the volume distribution. The non-monotonic $\mathcal{D}(V)$ explains the difference between the result of $\Gamma$ distribution model and the other two methods. It is also shown that $\mathcal{D}(V)$ gives insight on the definition of Random Loose Packing and Random Close Packing. 


\section{Part II}

\section{Packings in 3D}




\section{Chapter 6}

\section{Experimental Protocol}

The sphere packings are prepared for two kinds of experiments: dynamic heterogeneities $(\mathrm{DH})$ and statistics of the static packings. These two kinds of experiments are conducted in a water-fluidized bed but with different beads and fluidization condition. The 3D image of the bed was taken by X-ray tomography at ESRF (European Synchrotron Radiation Facility), Grenoble, France.

\subsection{Setup}

(a)

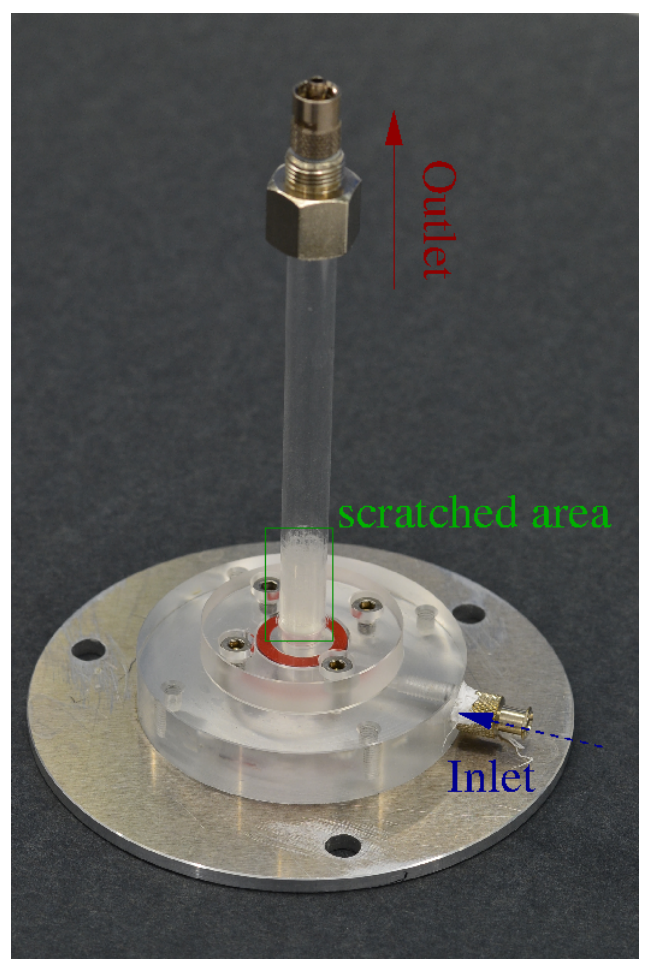

(b)

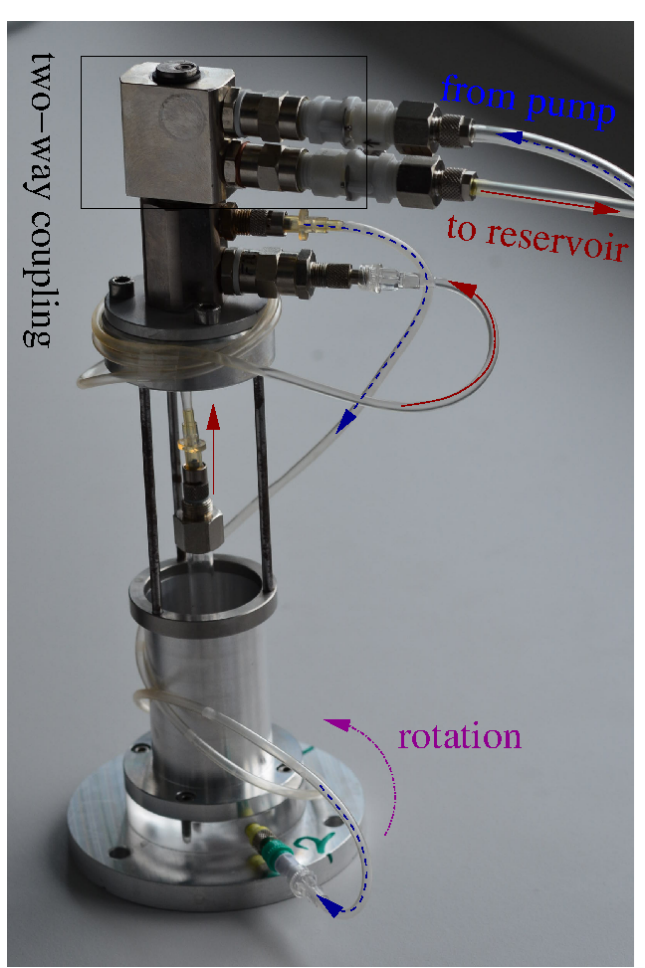

Fig. 6.1: (a): The tube used in the static packing experiments. The inner surface is scratched to $1 \mathrm{~cm}$ from the distributor. The incoming flow (blue dashed line) passes through a one-way valve at the bottom then through the distributor then through the packing and goes out of the tube via the top connector (red line). A filter inside the connector prevents the water flow from taking beads out of the tube. (b): The tube is mounted with the rotational two-way coupling. The in- and out-path are sketched in blue dashed line and red solid line respectively. The base of the setup is mounted on the translation-rotation stage in front of the camera. During the tomography the two-way coupling mounted on top is fixed while the lower part rotates (yellow dashed line).

Sphere packings are prepared in a tube with $8 \mathrm{~mm}$ inner diameter. The bed is put vertically and the degassed water flows through a distributor to fluidize the bed. The flow 


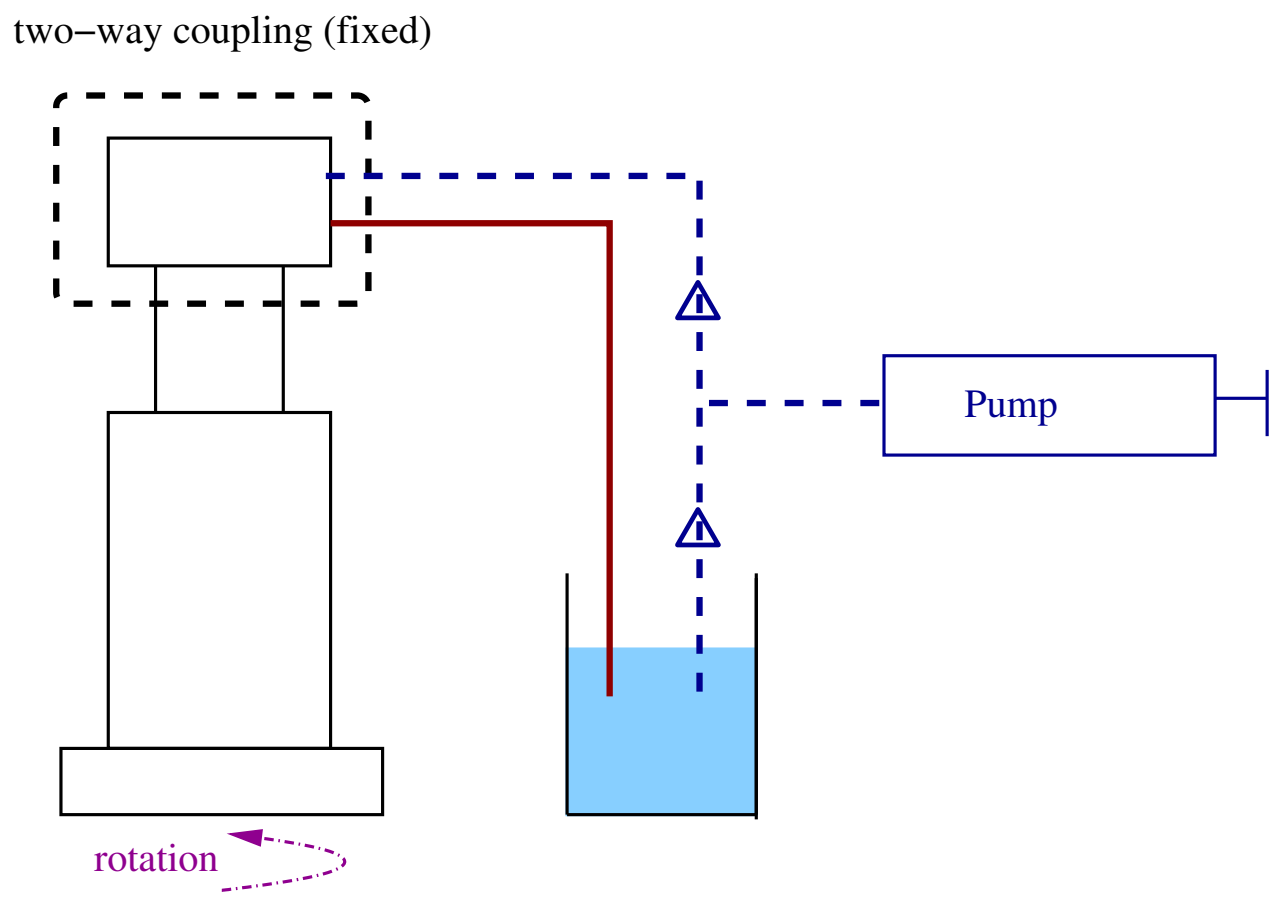

Fig. 6.2: The sketch of the experimental setup. The blue dash line and the red solid line represent the incoming and outgoing water flow respectively [cf. Fig. 6.1]. During the experiments, the two-way coupling (black dash rectangular) is fixed, and the tube is rotated with the stage. when the pump advances, water flows through a one-way valve to the two-way coupling. When the pump redraws, the water from the reservoir through another one-way valve refills the syringe. The one-way valves are denoted by the two solid blue triangles. The valves only allow the flow towards its vertex along the path.

rate and the duration are controlled by a customized syringe pump. Because the bed has to be rotated while taking a tomogram, the in- and out-connection of the flow of water have been realized through a rotational two-way coupling mounted on top of the fluidized bed. The in- and out-connection of the two-way coupling are connected with the syringe pump and a water reservoir respectively. The flow paths are demonstrated in Fig. 6.1.

When fluidizing the bed, the pump advances and pushes the degased water through the two-way coupling then through the distributor at beneath the bed. After the fluidization the pump redraws, and water from the reservoir refills the syringe through another oneway valve [cf. Fig. 6.2].

\subsection{Static packings}

The static packings are composed of 14000 quartz beads with a diameter $351 \pm 0.5 \mu \mathrm{m}$. The inner surface of the tube is scratched manually to prevent crystallization near the boundary [cf. Fig. 6.1(a)].

The bed is expanded by a short water flow then it has 10 seconds to sediment. A tomogram is taken while the bed is static, and the cycle is repeated. Each cycle takes 90 seconds. The sequence is controlled by a Labview program which reads trigger signal directly from the camera. We subtract two consequent tomograms with a flow pulse in 
Tab. 6.1: The flow rates and durations used to generate three static packing experiments. The packing fraction $\phi$ is computed by averaging their Voronoi volumes. Details can be found in Chapter 8 .

\begin{tabular}{|ccccc|}
\hline packing fraction $\phi$ & flow rate & flow duration & number of taps & resolution \\
\hline 0.5755 & $250 \mu \mathrm{l} / \mathrm{s}$ & $10 \mathrm{~s}$ & 307 & $12 \mu \mathrm{m}$ \\
0.5772 & $167 \mu \mathrm{l} / \mathrm{s}$ & $10 \mathrm{~s}$ & 324 & $12 \mu \mathrm{m}$ \\
0.5844 & $150 \mu \mathrm{l} / \mathrm{s}$ & $15 \mathrm{~s}$ & 516 & $18 \mu \mathrm{m}$ \\
\hline
\end{tabular}

between to make sure that the flow rate is sufficient to alternate the bed. We also test two tomograms without flow pulse to make sure that the rotation of the bed while taking the tomogram does not disturb the bed. The fluidized parameters used in three experiments and the number of cycles are listed in Tab. 6.1.

\subsection{Dynamic Heterogeneities}

The DH experiment is performed in the same setup but with $250 \mu \mathrm{m}$ diameter soda-lime glass beads. Three inner surface conditions: smooth (untreated), manually scratched and glued a layer of the same beads are applied in the experiment. The bed is prepared with a primary and several secondary pulse: ${ }^{1}$ then it is fluidized by a constant flow rate in the range between 33 and $46.5 \mu \mathrm{l} / \mathrm{s}$.

(a)

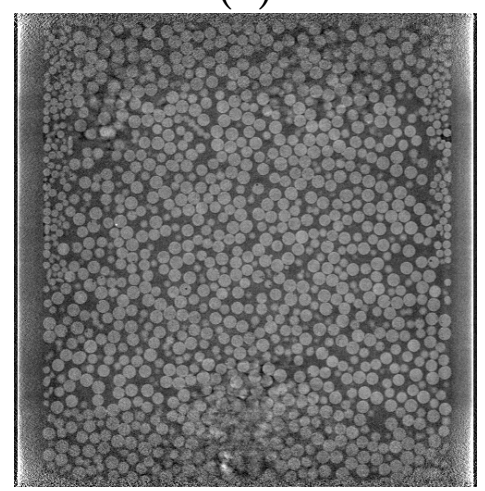

(b)

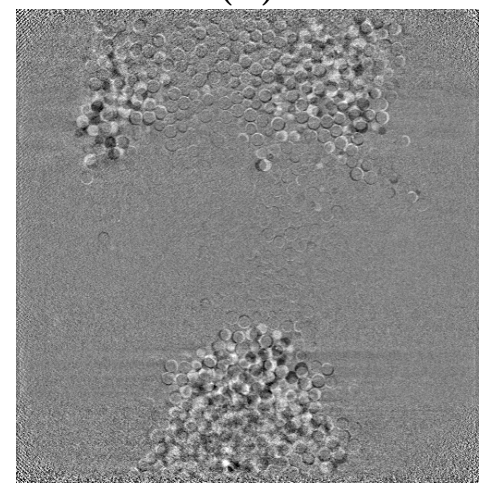

(c)

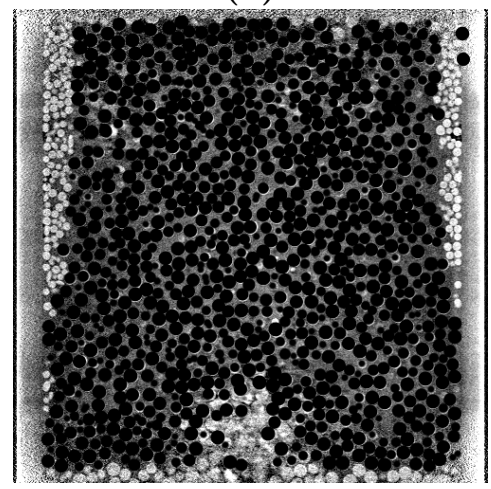

Fig. 6.3: Reconstruction image of tomograms. (a): A $x z$ cross-section of the bed fluidized with a flow rate of $38 \mu \mathrm{l} / \mathrm{s}$. (b): The difference of two subsequent tomograms which are taken with a time interval $\Delta t \approx 3.5 \mathrm{~s}$. Moving particles appear in black and white. (c): Corresponds to panel (a) after the beads detected by image processing have been removed by black.

Aim of this experiment is to study the formation and evolution of dynamical heterogeneities with the help of fast tomography. The normal tomography taking progress starts with moving the sample out of focus and taking a flat image, then sample is moved back and is rotated $180^{\circ}$ to take a tomogram, finally the sample is rotated back and the image data is transferred from the camera buffer to the storage hard drive. To achieve fast

\footnotetext{
${ }^{1}$ The typical preparation is started with a flow $67 \mu l / s \times 2 m l$ then is followed by several $1 m l$ pulses with descending flow rate step $\sim 8 \mu l / s$.
} 
tomography only the first flat image is taken and the sample is rotated continuously to take continuous tomograms. In this fast mode the time interval between two consecutive tomograms is just the exposure time, typically 1.75 seconds. The typical duration of one experiment is 6 minutes, which approximately corresponds to 160 frames.

Fig. 6.3 (a) shows a cross-section through a fluidized bed where the beads are driven with a flow rate of $38 \mu \mathrm{l} / \mathrm{s}$. The motion blur visible in the center bottom part indicates that the exposure time $1.75 \mathrm{~s}$ was not short enough to freeze the motion of the fastest beads. Panel (b) of the same figure shows the difference image between two subsequent tomograms of the same experiment. There exist three clearly separated regions where the beads are moving, while the rest of the bed is virtually at rest, thus demonstrating for the existence of $\mathrm{DH}$ in three dimensions. However, the detection of particles fails for the fast moving region. Therefore the quantitative analysis of the motion of particles is not successful. 


\section{Chapter 7}

\section{Image Processing}

The tomograms are taken for experiments with various projection numbers and exposure times, hence the image quality varies. This chapter mainly introduces the image processing of the static packings experiment. In practise, some modifications (e.g., the parameters referred to below and the binary threshold algorithm) are applied according to the resolution and image quality. For convenience $z$ denotes the anti-gravity direction and the cross-section in the $x y$ plane is referred to a slice. The region of interest, abbreviated as ROI henceforth, is within the tube [cf. Fig. 7.1(a)]. The detection of a sphere follows the same idea as that of discs: segmentation by binarization and erosion, finally convolution correction. However, the binarization and convolution implementation are heavily modified from the $2 \mathrm{D}$ version.

\subsection{Binarization}

In some tomograms the pixels outside the ROI are negatively valued [cf. Fig. 7.1(b)]. Those pixels skew the Otsu's algorithm giving a too low threshold [cf. Fig. 7.1(a)]. On the other hand, the intensity within the ROI is not as homogeneous as that of disc packing. For example, the right half of the slice displayed in Fig. 7.1(a) is brighter than the left half. To correct for this, binarization is done locally by the following steps.

The tomogram is first treated with median filter with a 3 by 3 pixel neighbourhood. This filter replaces the value of a pixel with the media value of its neighbours. This reduces the random noise. Then a global threshold is computed with Otsu's algorithm by using only the voxels contained within the ROI. This global threshold is not applied to binarize the tomogram directly but is used as a reference.

Binarization is processed slice by slice along the $z$ direction. The working slice is divided into evenly spaced $60 \times 60$ pixel square regions. For each such square region a local threshold is calculated and is compared with the global one. If the difference is less than 0.1, the local threshold is used to binarize that square, otherwise the global threshold is applied and a message about the position of that local region is given. The local threshold is supposed to optimize the threshold for small variances in intensity. According to my experience the large deviation of the local threshold to the global one merely happens at the top and bottom of the tomogram where the noise is strong. After the same process is applied to all slices a three dimensional binary image is obtained which is referred to as bw_map henceforth. 
(a)

raw tomogram

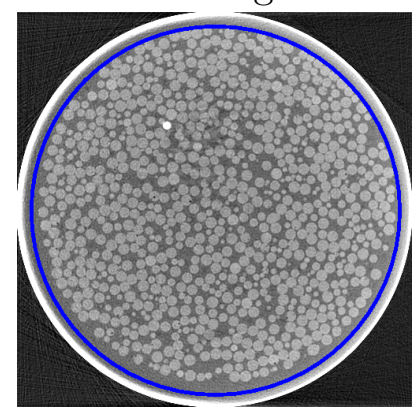

Otsu's algorithm globally

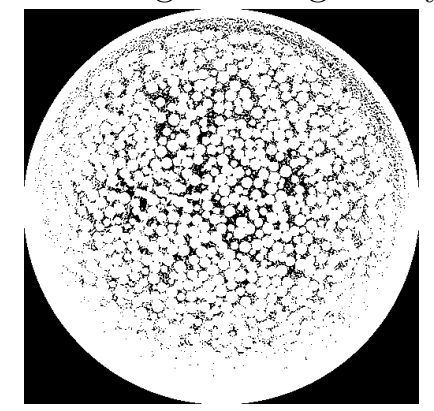

local binarization

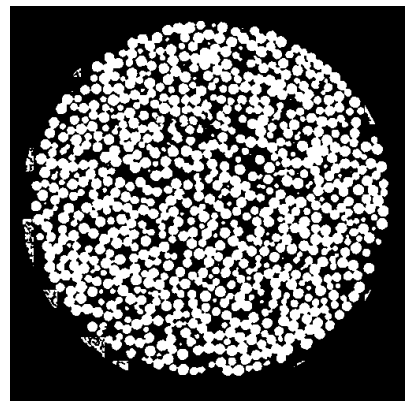

(b)

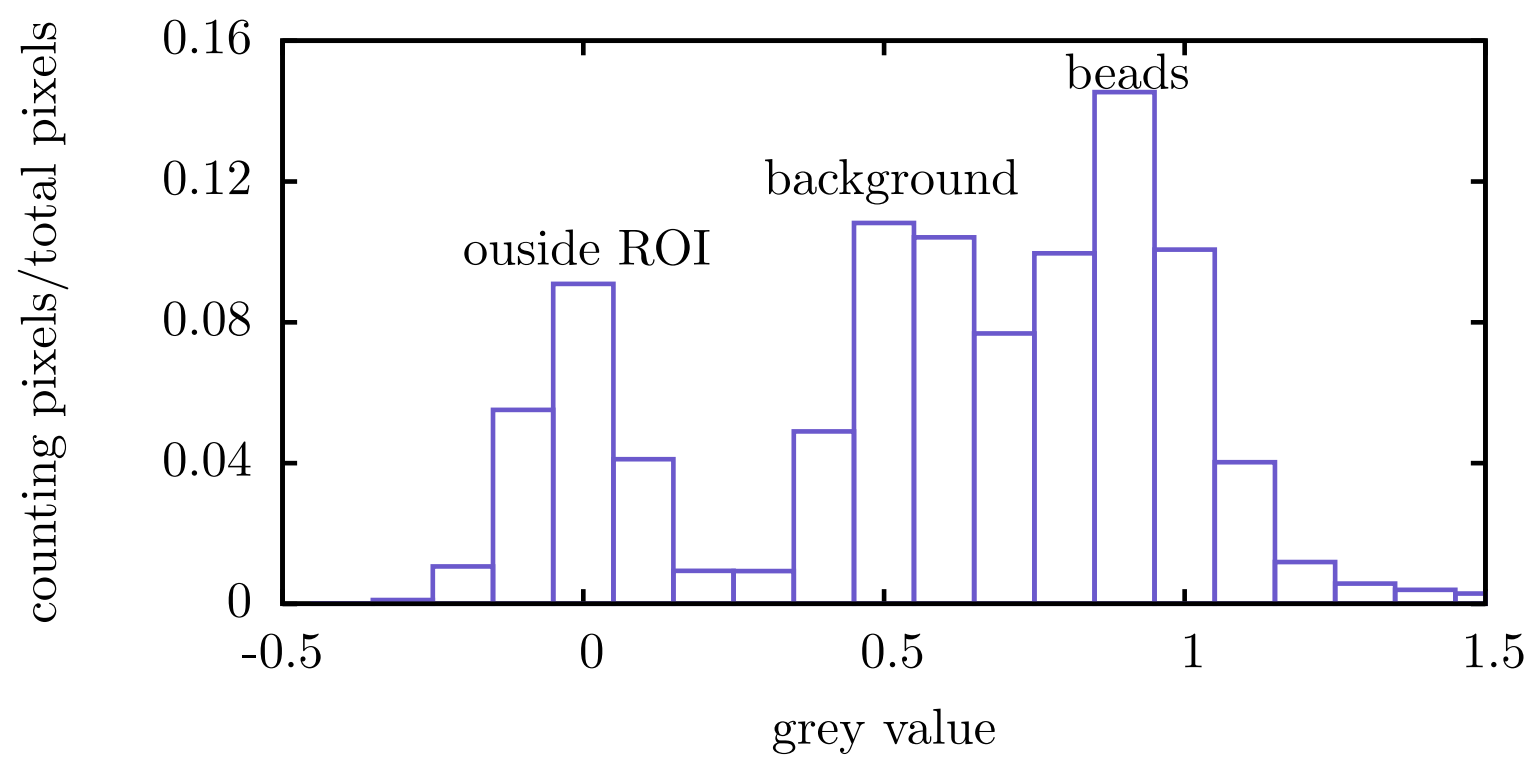

Fig. 7.1: (a): From left to right: one slice of a raw tomogram, the blue circle indicates the region of interest (ROI); the same slice binarized according to the threshold computed by Otsu's algorithm globally; the same slice binarized by applying Otsu's algorithm in $60 \times 60$ pixel $^{2}$ squares tiling the ROI. (b): The histogram of the grey value of the slice of the raw tomogram in (a). The pixel values have three domains: the pixels outside the ROI, the background pixels and the beads pixels. 

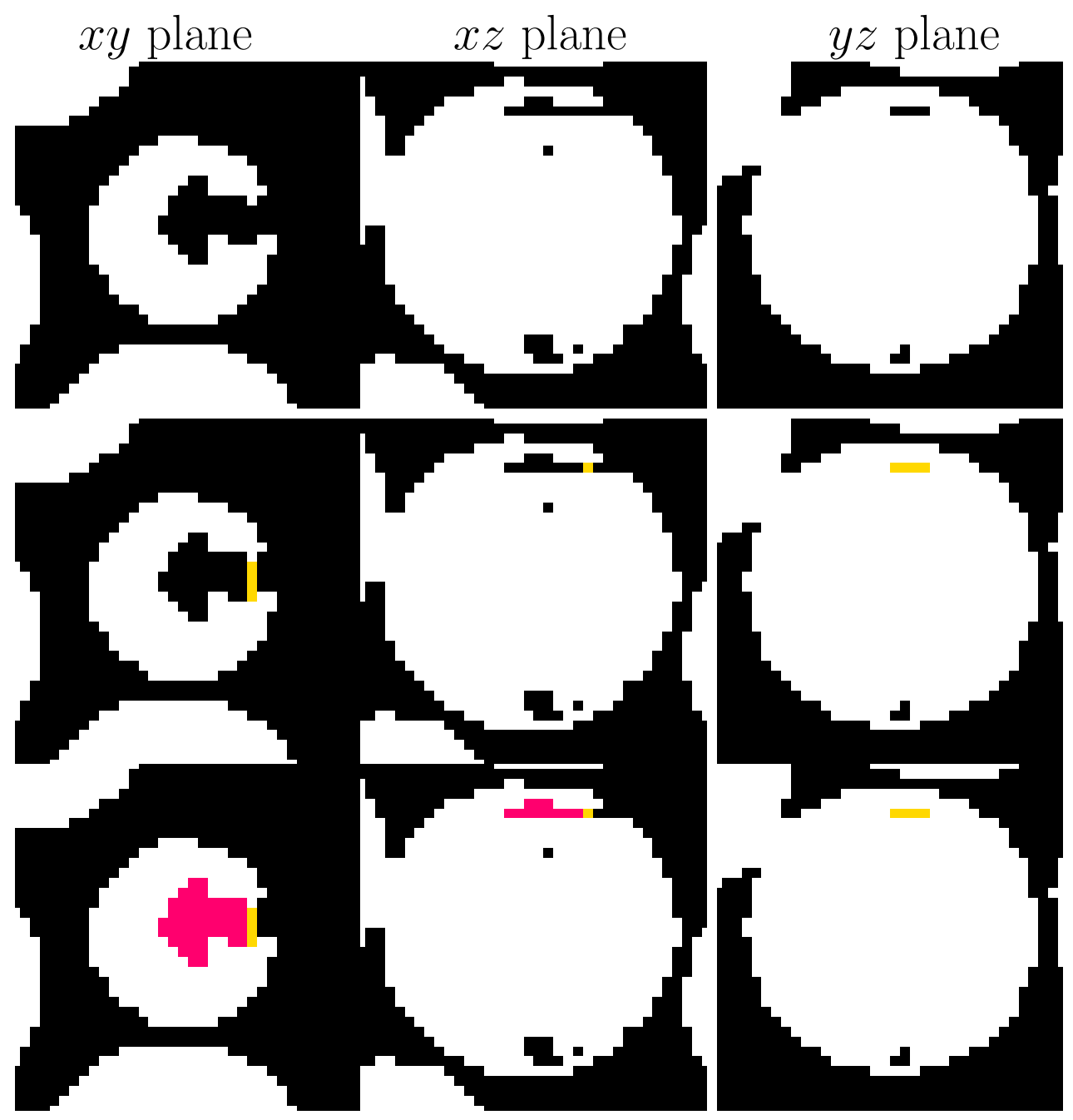

Fig. 7.2: This demonstration exhibits the sequential progress of removing only the defect near the top ( $z$ direction) of a bead. In practise other defects are removed by a similar process. ToP ROw: the projection of the defect on $x y, x z, y z$ plane. MIDDLE ROw: The defect is removed according its $y z$ projection and the corresponding changes on $x y, x z$ projections. In this step the changed pixels are coloured yellow, in practise they are changed to white. воттом Row: the remaining defect pixels are now closed and removed. The corresponding changes on $x y, x z$ projections are coloured pink. 


\subsection{Defects removal}

The glass beads have voids and defects which may be recognized as black objects (pixel clusters) during the binarization [cf. Fig. 7.2]. Such defects could easily 'break' a bead after the erosions, so the removal of defects should be done prior to the erosion step.

We distinguish between two categories of defects. Some defects are completely inside the beads, or in other words are completely separated from other black objects by white pixels. This kind of defects can be easily detected and removed ${ }^{1}$ in three dimensions, so called 3D-MODE DEFECTS. The other defects are connected to the background by 'touching' the beads' surface. In practise such a touching area is a small hole. By changing the view angle this small hole may be detected and removed on one of its 2D projections. Then the whole defect is transformed into the 3D-MODE DEFECTS. According to the detection methods this kind of defects is called 2D-MODE DEFECTS. Following this idea $b w \_m a p$ is firstly checked for 2D-MODE DEFECTS slice by slice along all three dimensions then all 3D-MODE DEFECTS are detected and removed.

Fig. 7.2 demonstrates this progress in detail. A defect is observed near the top (along the $z$ direction) of the bead. This defect connects the background pixels according to its $x y$ and $x z$ projections but is surrounded by white pixels in the $y z$ projection. Then the defect pixels in the $y z$ projection are removed first [cf. Fig. 7.2 Middle row]. The corresponding changes on $x y$ and $x z$ planes are displayed in Fig. 7.2 Middle row. The whole defect is 'closed' and can be removed [cf. Fig. 7.2 Bottom row].

\subsection{Erosion and centroid detection}

The erosion algorithm is applied to bw_map to separate white objects which would be identified as beads. Depending on the quality of the tomogram the previous step may not remove all defects, so the erosion should be done with small erosion matrix twice sequentially rather than once with a large erosion matrix. After the first erosion the objects whose volume are in the range $20 \% \sim 40 \%$ of the bead volume $v$ are identified as beads and are taken out of bw_map. The objects below this range are identified as noise and are taken out as well. The remaining objects are left for the second erosion. Finally after the second erosion the objects which are between $14 \% \sim 28 \%$ of $v$ are beads. Those larger than this threshold are abandoned. For all detected beads the centroid are located.

If the applied erosions do not separate the beads sufficiently, the connected bead objects would be too large to be detected. To quantitatively examine the completeness of the detection, a spherical mask centring each centroid is generated on the raw tomogram [cf. Fig. 7.3]. The masked tomogram goes through the binarization steps. If there are remaining objects, the positions of those are reported. It is found that all missing beads are on the top and bottom layers in which the beads are not fully captured by the tomogram and the pixels are noisy.

If the defects in the beads are not removed efficiently, the erosion may induce another artefact where the bead is cracked into two or even more pieces by the erosions. If the threshold of the detection after the erosion is not large enough, the pieces may result in

\footnotetext{
${ }^{1}$ Here being removed means that the black pixels are changed to white.
} 

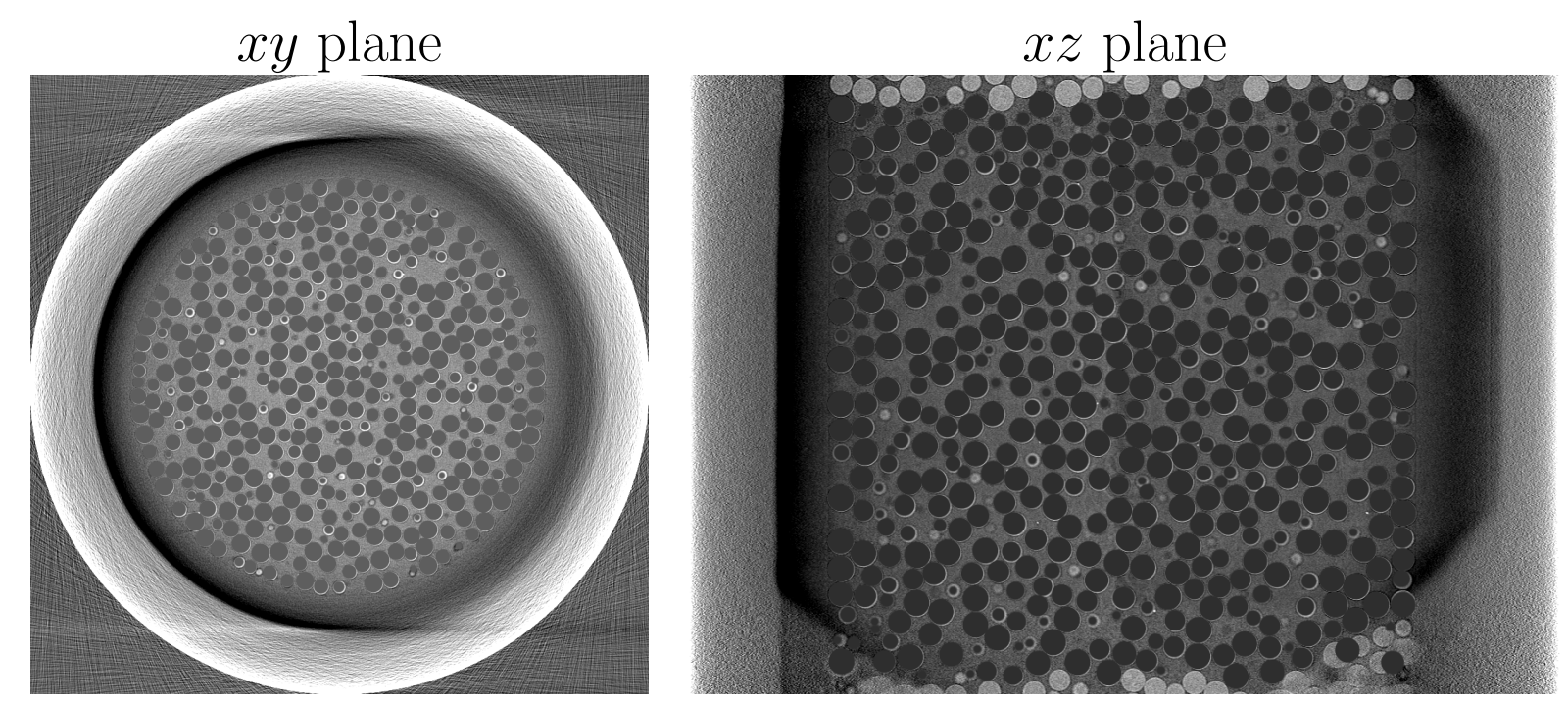

Fig. 7.3: Cross sections on $x y$ plane and $x z$ plane respectively. The detected beads are masked with a black pixels.

being recognized as individual beads. To quantify these possible artefacts we compute the pair correlation function $g(r)$ and accumulate the amount of pairs with $r<0.5 d_{s}$ and list the locations of those pairs. Those short $r$ pairs are very rare and are merely located in the first layer of the cylindrical boundary, because of the large intensity variance near the boundary.

\subsection{Convolution correction}

For a given packing, the width of the first peak of $g(r)$ indicates the polydispersity and the accuracy of the centroid detection. As stated in chapter 6, the sphere packings are composed of different kinds of beads for the different experiments. For the soda-lime beads the polydispersity dominates the width of the peak. A series of templates with different sizes are convoluted with every detected bead. The size of a bead is determined by the template that gives the largest normalized convolution value and the centroid is tuned accordingly. The resulting $g(r)$ has a narrower peak [cf. Fig. 7.4(a)].

The quartz beads are less polydisperse. The centroids detected by the segmentation already give a very sharp peak in $g(r)$ [cf. Fig. 7.4(b)]. We perform the convolution with a fixed size template to finely tune the center detection in the same way as for the disc packings [cf. Chapter 3. $g(r)$ is computed both with and without convolution correction and plotted in Fig. 7.4(b). Intuitively the erosion can't be isotropically performed on a given bead because of its neighbours within the vicinity. On the other hand, the convolution is performed on pixel level and fitted to get sub-pixel estimation, which should give a better estimation of the centroid. Surprisingly we found that the convolution gives a wider peak. Careful study shows that the two groups of centroids make no difference on pixel level but the segmentation alone gives a better sub-pixel estimation.

To surpass the estimation of the centroids by segmentation one has to perform the native sub-pixel convolution. The straightforward algorithm is as follows: first locate the 


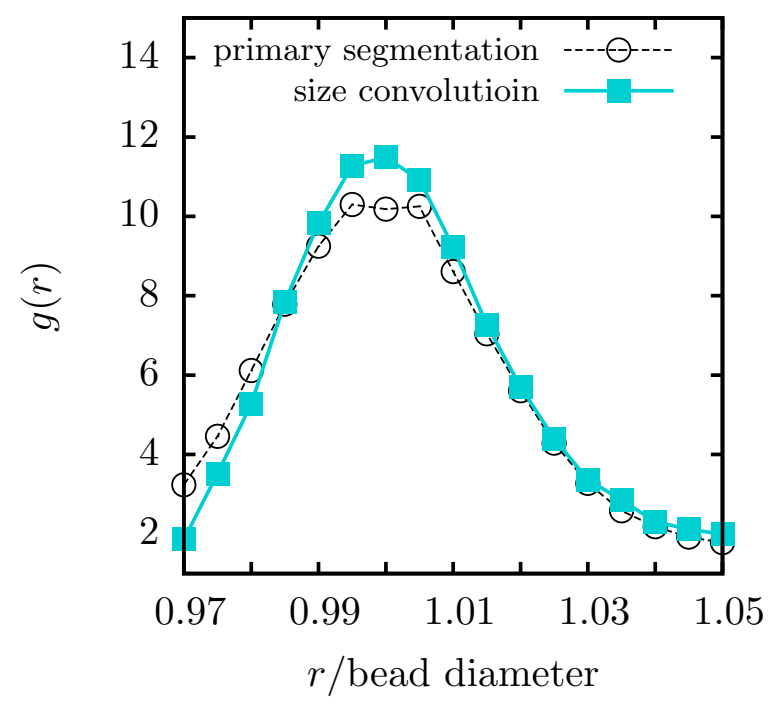

Fig. 7.4: $g(r)$ comparison between different sets of centroids. (a): For the DH experiment the solid squares are computed from the centroids with the size correlation correction. Open circles are computed from the segmentation results. (b): For the static packing the solid squares and triangles are computed from the sub-pixel and pixel convolution algorithm respectively. The open circles are computed from the segmentation results.

centroid of segmentation on pixel level, then create a series of templates shifted from that centroid with step 0.1 pixel along each dimension in a range from -1 to 1 pixel and do the convolution respectively, finally find the maximum convolution value and fit around it to get the correction to the centroid. The $g(r)$ computed from the centroids with this sub-pixel convolution is plotted in Fig. 7.4(b) with the other two methods. The sub-pixel convolution does give a narrower $g(r)$. The half-height width of the first peak of $g(r)$ with sub-pixel convolution is comparable to the small polydispersity of the quartz beads (0.0014 bead diameter). The small polydispersity is neglected in further analysis. 


\section{Chapter 8}

\section{Static sphere packings}

In this chapter we will apply the analysis tools introduced in Chapter 4 and Chapter 5 to static sphere packings to measure the correlation of Voronoi volumes and the configurational temperature.

\subsection{Voronoi volume distribution}

In Chapter 7 we see that the detection of beads fails near the top and bottom of the tomograms, and near the inner wall of the tube. The largest volume without those artefacts should be found first. We first compute the averaged packing fractions in a disk layer [cf. inset of Fig. 8.1(a)]. The packing fraction $\phi=V_{g} / V$, where $V_{g}$ is the total volume of beads, and $V$ is the total Voronoi volume. Voronoi volume is computed by voro++ package [Rycroft et al., 2006].

In Fig. 8.1 (a) we plot the packing fraction $\phi$ versus the height from the bottom $z$. The height for which $\phi$ is steady is identified from this plot. Then within this range of height, the averaged packing fraction of a cylindrical region is computed [cf. inset of Fig. 8.1(b)]. The axis of the cylindrical region is aligned with that of the experimental tube. In Fig. 8.1(b) we plot $\phi$ in the cylindrical region versus the radius $\rho$.
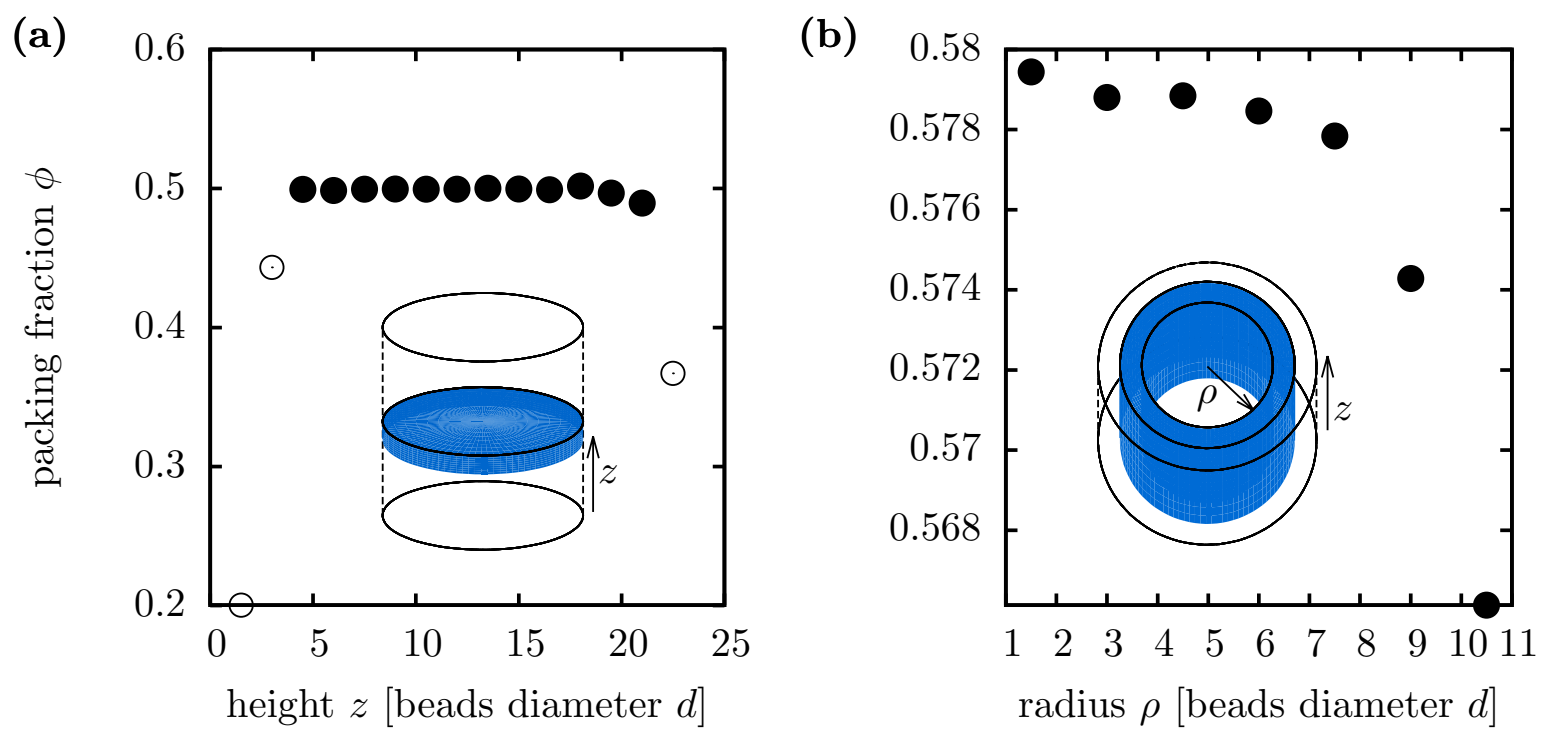

Fig. 8.1: (a): the packing fraction $\phi$ with height $z$. Packing fractions are computed by averaging Voronoi volumes within a disk layer (blue disk in the inset). The diameter of the layer is the diameter of the fluidized bed. The thickness of the layer is $1.5 d$, where $d$ is the bead diameter. Height $z$ denotes the distance to bottom of the tomogram. Open circles indicate where the image processing fails to detect all beads. (b): the packing fraction $\phi$ of a cylinder with radius $\rho$ (blue region in the inset). The width of the cylinder is $1.5 d$. The cylinder extends to the height range measured from (a). 


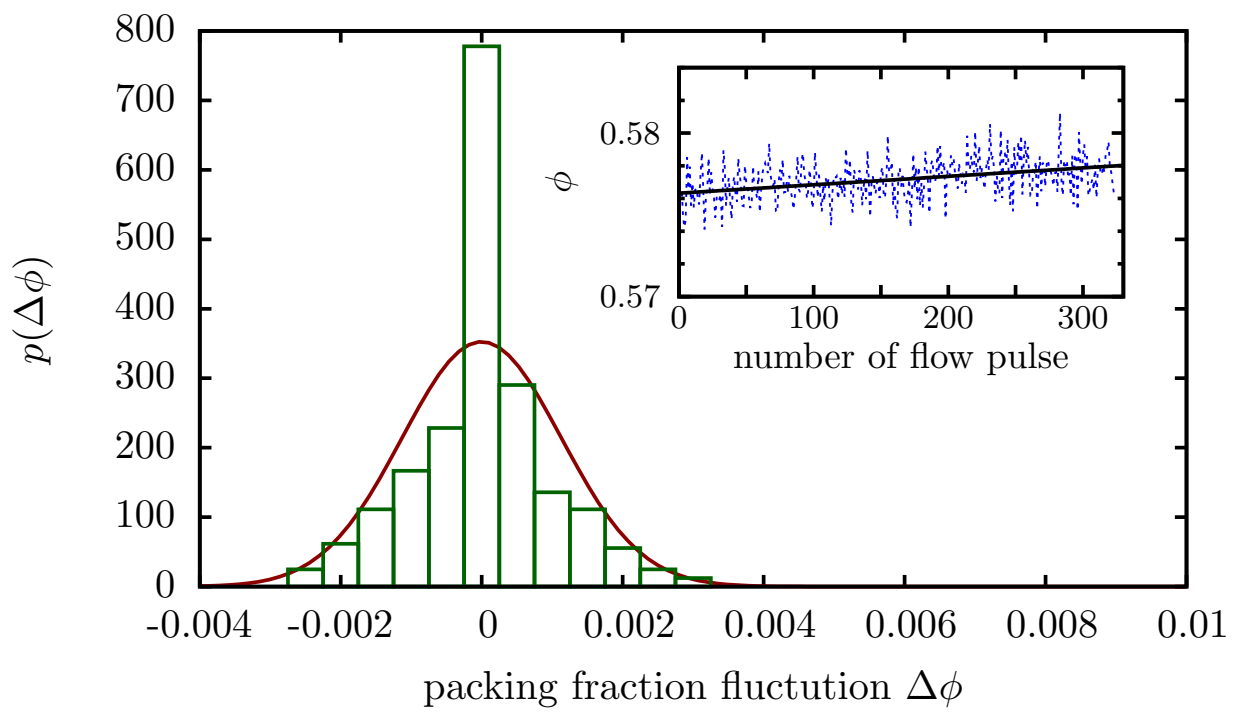

Fig. 8.2: INSET: packing fraction $\phi$ versus number of flow pulse (blue dash line). The black solid line is the linear fitting $\Phi$. MAIN PLOT: Histogram of the fluctuation $\Delta \phi$ (green boxes). The fluctuations are computed as $\Delta \phi=\phi-\Phi$. The red curve is a Gaussian distribution with the same mean and variance as those of the histogram.

In Fig. 8.1(a) we can see that at $z<5 d$ and $z>20 d$ the packing fraction is lower than 0.4 (open circles). In those regions the image processing starts to lose the detection of beads due to the image noises [cf. Chapter 7]. In Fig. 8.1(b) the packing fraction decreases while approaching the boundary. The radius of the experimental tube is approximately 12d. In 2D disc packings the local configuration are more ordered near the confinement walls Desmond and Weeks, 2009]. In our experiments the decreases of $\phi$ with $\rho$ may be due to the scratched wall [cf. Chapter 6]. We apply similar procedure on all three experiments to identify the $z$ and $\rho$ range as the analysis region.

Within the region of $z$ and $\rho$ identified, we compute the packing fraction after each flow pulses. We found that the packing fractions increases slightly with the number of flow pulses [cf. inset of Fig. 8.2]. A straight line $\Phi$ is fitted to get the slope of the increases of $\phi$. The probability of the fluctuation around $\Phi, \Delta \phi=\phi-\Phi$, is plotted in Fig. 8.2. The fluctuation is Gaussian like indicating that flow pulses are independent. The middle point of the fit $\Phi$ is taken as the averaged packing fraction $\phi_{\text {avg }}$. The volumes of the particles are corrected as $v-\frac{1}{\Phi}+\frac{1}{\phi_{\text {avg }}}$.

\subsection{Correlation between Voronoi volumes}

The correlation of Voronoi volumes is measured with the same technique in 2D packings [cf. Chapter 4]. A few hexagonal wheels are placed in the $x y$ plane along the central axis paralleling each other [cf. inset of Fig. 8.3. The distance between neighbouring wheels is 3 bead diameters. The arm length of the wheel is $L$, and each hexagonal wheel is translated to five different positions within the $x y$ plane and rotated to four different angles for each position. For a given $L$ the two-point correlation function, $C_{i j}(L)=\frac{\left\langle\left(v_{i}-\bar{v}_{i}\right)\left(v_{j}-\bar{v}_{j}\right)\right\rangle}{\sigma^{2}}$, is first 


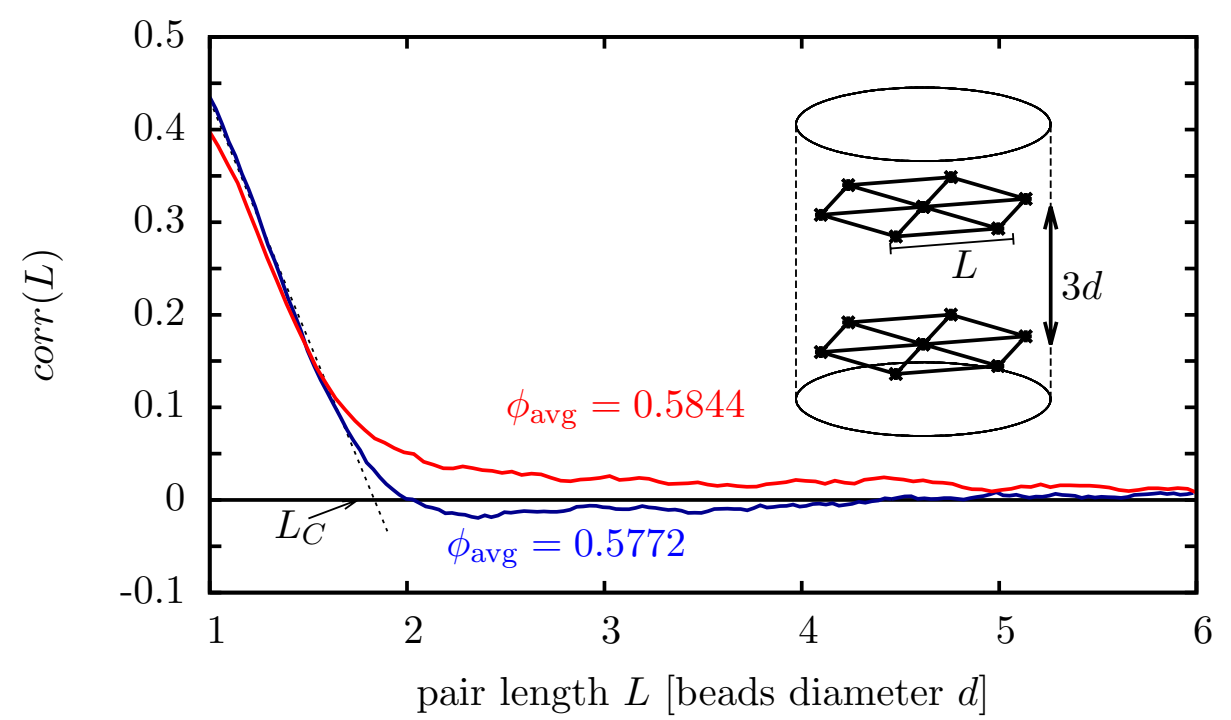

Fig. 8.3: Correlation between Voronoi volumes, $\operatorname{corr}(L)$, is measured in the hexagonal wheels in the $x y$ plane like that in the inset. The wheel side length is $L$. The space between those wheels is 3 bead diameters. $\operatorname{corr}(L)$ for packing $\phi_{\text {avg }}=0.5772$ (blue) and 0.5844 (red) are displayed. See text for details.

computed in each whee ${ }^{1}$, then are averaged over wheels:

$$
\operatorname{corr}(L)=\frac{1}{N_{\text {pair }}} \sum_{\text {wheels }} \sum_{i, j} C_{i j}(L) .
$$

See Fig. 8.3 for results.

As in $2 \mathrm{D}$ packings, $\operatorname{corr}(L)$ decays from a maximum. The maximum is two times larger than that in $2 \mathrm{D}$ packings. In $2 \mathrm{D}$ packings, we have shown that anti-correlation happens in dense packings and becomes stronger with packing fraction [cf. Fig. 4.16]. Nevertheless, weak anti-correlation is observed for $\phi_{\text {avg }}=0.5755$ and 0.5772 , but not for the densest bed $\phi_{\text {avg }}=0.5844$ [cf. Fig. 8.3].

We extract the length $L_{C}$ where the straight line fit crosses the zero [cf. Fig. 8.3], and $L_{\min }$ where the negative minimum is. We also integrate the weighted area $A$ under $\operatorname{corr}(L)$ from $d$ to $6 d$. This area is defined as:

$$
A=4 \pi \varrho \int_{d}^{6 d} \operatorname{corr}(L) L^{2} \mathrm{~d} L .
$$

$\varrho$ is the density. $4 \pi \varrho L^{2} \mathrm{~d} L$ gives the number of particles within the spherical shell from $L$ to $L+\mathrm{d} L$. The results are listed in Tab. 8.1.

$L_{C}$ increases with packing fraction. This length characterizes the positive correlation. The larger $L_{C}$ is, the stronger the positive correlation between fluctuation of local volumes. As discussed in Chapter 4 , the increases of $L_{\text {min }}$ implies the same tendency. The

\footnotetext{
${ }^{1}$ where $i, j$ correspond to two points in a distance $L$ belonging to two Voronoi volumes, and $v_{i}, v_{j}$ are the free volume of these Voronoi cells. $\langle\ldots\rangle$ indicates averages over all packings created by flow pulses. $\bar{v}_{i}$ and $\bar{v}_{j}$ are the mean free volumes at these points, and $\sigma^{2}=\left(\sigma_{i}^{2}+\sigma_{j}^{2}\right) / 2$ is the corresponding variance.
} 
Tab. 8.1: Quantities extracted from $\operatorname{corr}(L), L_{C}$ is where the linear fit equals zero [cf. Fig. 8.3]. $L_{\min }$ is the position of the negative minimum. $A$ denotes the area under $\operatorname{corr}(L)$. The range of $L$ for the computation of $A$ is from $d$ to $6 d$, where $d$ is the bead diameter.

\begin{tabular}{|cccc|}
\hline$\phi_{\text {avg }}$ & $L_{C} / d$ & $L_{\min } / d$ & $A / d^{3}$ \\
\hline 0.5755 & 1.760 & 2.2606 & 2.52 \\
0.5772 & 1.763 & 2.3604 & 2.04 \\
0.5844 & 2.053 & - & 21.78 \\
\hline
\end{tabular}

correlation curve $\operatorname{corr}(L)$ for $\phi_{\text {avg }}=0.5844$ does not decay to zero rapidly beyond $L_{C}$, but has a long tail. The tail of $\operatorname{corr}(L)$ beyond $L_{C}$ has a large contribution to the weighted area $A$, such that $A$ of the densest bed is more than 5 times larger than that of the other two packing fractions [cf. Tab. 8.1]. As discussed in Chapter 5, the weighted area $A$ quantify the contribution of correlation between local volumes to the fluctuation of the volume of the system. In $2 \mathrm{D}$ packings, this contribution decreases with packing fraction due to the appearance of anti-correlation. However, for 3D packings the denser bed has a stronger correlation. This unexpected feature of correlation can be used to explained the fluctuation of the volume with packing fraction in the next section.

\subsection{Compactivity measurement}

In this section we demonstrate the compactivity (configurational temperature) measurement by the overlapping histogram and the fluctuation dissipation theorem for different cluster sizes $N$. We start with the overlapping histogram.
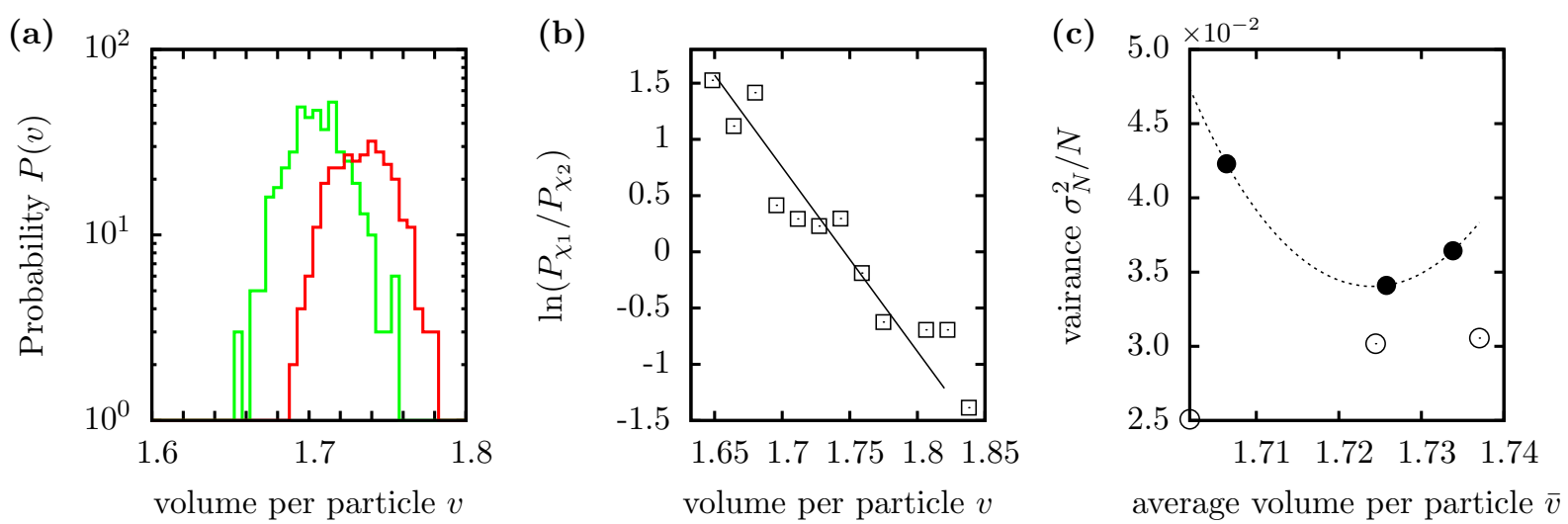

Fig. 8.4: Demonstrations of the methods of the overlapping histogram and fluctuation dissipation theorem. (a): The distribution of volume per particle $v$ of $\phi_{\text {avg }}=0.5844$ and 0.5755 for cluster size $N=100$. (b): The ratio of the distributions in (a). The solid line is the straight line fit. The corresponding slope is extracted as the difference of the inverse compactivity $\Delta \frac{1}{\chi_{O H}}$. (c): The variance per particle $\sigma_{N}^{2} / N$ versus the averaged volume per particle $\bar{v}$. The dash line is a parabolic fit which is used in the Eq. 8.4 to computed $\Delta \frac{1}{\chi}$.

Fig. 8.4(a) and (b) display two distribution and their overlapping ratio. As what we did in 2D packings [cf. Chapter 5] a straight line is fitted to the logarithm of the 


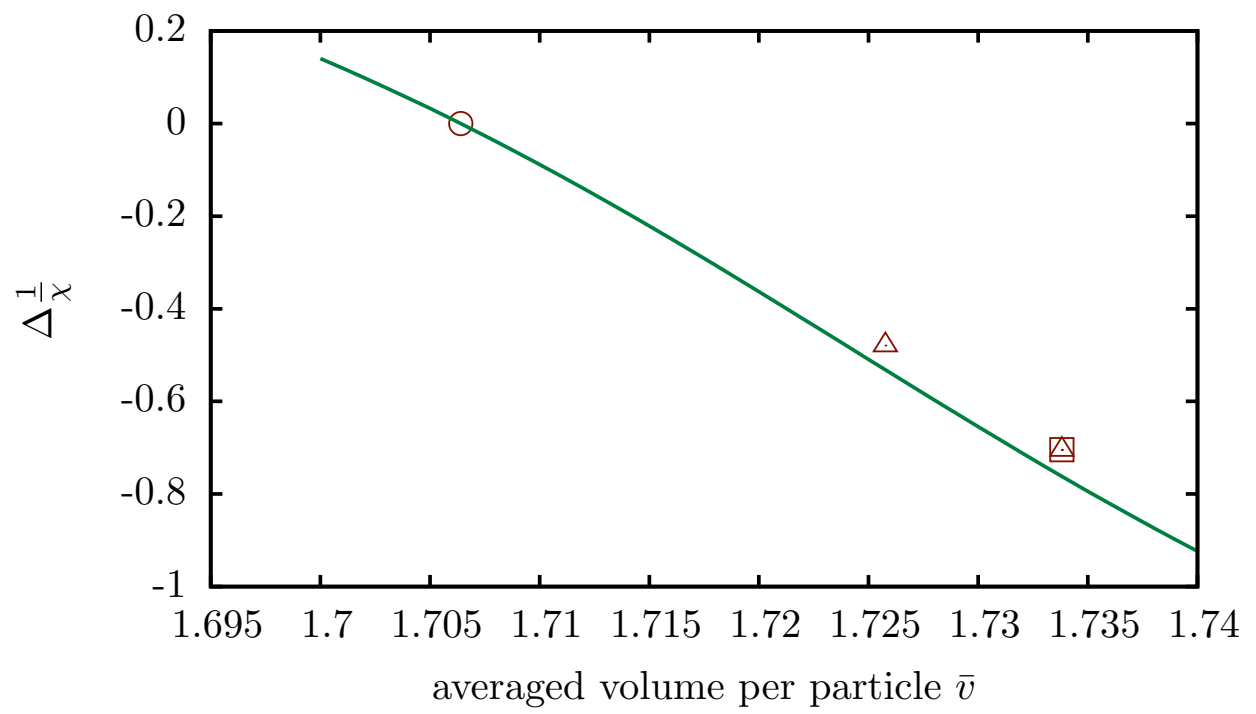

Fig. 8.5: The resulting difference of the inverse compactivity $\Delta \frac{1}{\chi}$ of the overlapping histogram method (points) and the fluctuation dissipation theorem (solid line) for the cluster size $N=$ 100. The open circle is the reference $\left.\frac{1}{\chi_{O H}}\right|_{1}=0$. The two triangles correspond $\left.\Delta \frac{1}{\chi}\right|_{2,1}$ and $\left.\Delta \frac{1}{\chi}\right|_{2,1}+\left.\Delta \frac{1}{\chi}\right|_{3,2}$ respectively. The square is $\left.\Delta \frac{1}{\chi}\right|_{3,1}$ which should equal the triangle point at the same $\bar{v}$ in ideal case. See text for details. The solid line is computed by the integral of fluctuation-dissipation theorem [cf. Eq. 8.4].

ratio and the slope is extracted as the difference of inverse compactivity between the two packing fractions. The three datasets are ordered in ascending order according to the averaged volume per particle $\bar{v}=1 / \phi_{\text {avg }}: \bar{v}_{1}=1.712, \bar{v}_{2}=1.7325$ and $\bar{v}_{3}=1.7376$. All possible differences, $\left.\Delta \frac{1}{\chi_{O H}}\right|_{2,1},\left.\Delta \frac{1}{\chi_{O H}}\right|_{3,2}$ and $\left.\Delta \frac{1}{\chi_{O H}}\right|_{3,1}$ are calculated, where $\left.\Delta \frac{1}{\chi_{O H}}\right|_{a, b}=$ $\left.\frac{1}{\chi_{O H}}\right|_{a}-\left.\frac{1}{\chi_{O H}}\right|_{b}$. The results are plotted in Fig. 8.5. In the plot $\left.\frac{1}{\chi_{O H}}\right|_{1}$ is set to be 0 (the open circle). The two triangle points are the accumulation of the difference measured between neighbouring $\bar{v}$, and the square point is the difference, $\left.\Delta \frac{1}{\chi_{O H}}\right|_{1,3}$, measured between $\bar{v}_{1}$ and $\bar{v}_{3}$. In ideal case $\left.\Delta \frac{1}{\chi}\right|_{2,1}+\left.\Delta \frac{1}{\chi}\right|_{3,2}=\left.\Delta \frac{1}{\chi}\right|_{3,1}$. We define the difference $\kappa$ as:

$$
\kappa^{2}=4\left(\frac{\left.\Delta \frac{1}{\chi_{O} H}\right|_{3,1}-\left.\Delta \frac{1}{\chi}\right|_{2,1}-\left.\Delta \frac{1}{\chi}\right|_{3,2}}{\left.\Delta \frac{1}{\chi_{O} H}\right|_{3,1}+\left.\Delta \frac{1}{\chi}\right|_{2,1}+\left.\Delta \frac{1}{\chi}\right|_{3,2}}\right)^{2}
$$

and compute it for different cluster sizes. The result is shown in Fig. 8.6. On average $\kappa$ is around 0.1 and decreases with cluster size $N . \kappa>0.2$ happens only below $N=200$. The histogram of larger clusters has better statistics, therefore the uncertainty is smaller, and the extraction of $\Delta \frac{1}{\chi_{O H}}$ from the ratio of two histograms is more reliable.

To apply fluctuation dissipation theorem the fluctuation of the volume should be computed first. For small clusters $(N<50)$ the fluctuation per particle $\sigma_{N}^{2} / N$ increases monotonically with averaged volume per particle $\bar{v}$, which indicates that in dense packing the fluctuation of local volume is smaller than that in the loose packings. However, we discovered that for large clusters $\sigma_{N}^{2} / N$ is larger in the dense packings $\left(\phi_{\text {avg }}=0.5844\right)$ than that in the loose packings [cf. Fig. 8.4(c)]. The same behaviour has been reported by Schröter et al. [2005]. The difference of the local and global fluctuation can be again 

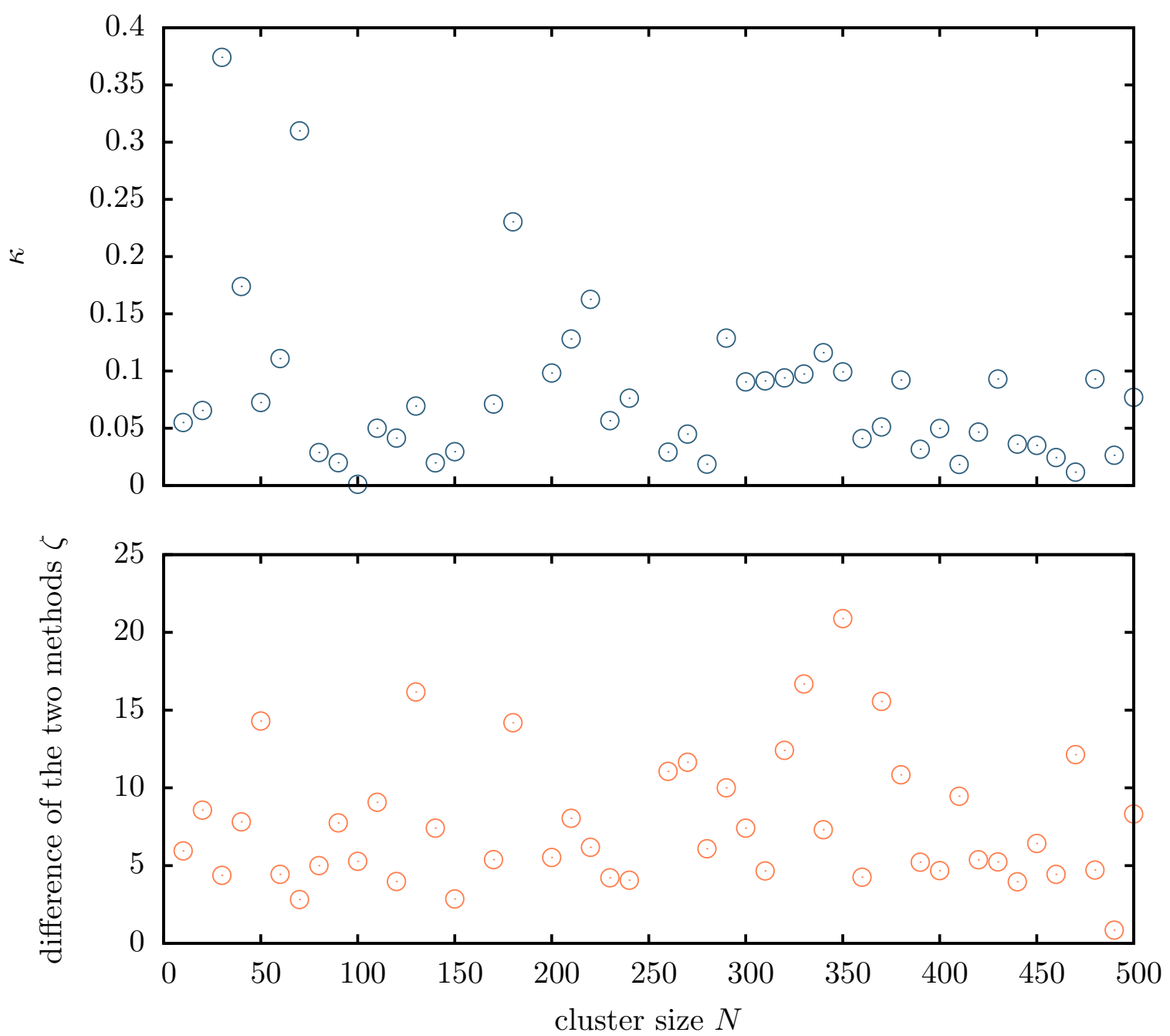

Fig. 8.6: TOP: The difference, $\kappa$, between $\left.\Delta \frac{1}{\chi_{O H}}\right|_{3,1}$ measured from the histograms of $\bar{v}_{3}$ and $\bar{v}_{1}$ and the accumulation of that measured from $\bar{v}_{3}$ and $\bar{v}_{2}$ and $\bar{v}_{2}$ and $\bar{v}_{1},\left.\Delta \frac{1}{\chi}\right|_{2,1}+\left.\Delta \frac{1}{\chi}\right|_{3,2}$. See Eq. 8.3 and the text for details. воттом: The difference of $\Delta \frac{1}{\chi_{O H}}$ and $\Delta \frac{1}{\chi_{F D T}}$ for different cluster sizes $N$. The detail of the computation is explained in the text.

related to the correlation between local volumes. Recall that the volume fluctuation of a cluster can be decomposed into the volume fluctuation of individual cells and the correlation between cells. Although the fluctuation of individual cells are smaller in dense packings, the correlation is stronger [cf. Tab. 8.1]. With increasing cluster size, the contribution of correlation compensates and overweights the decreasing fluctuation of individual cells, and leads to a larger fluctuation of the volume of the cluster in the dense packing.

We fit the variance $\sigma_{N}^{2}$ versus $\bar{V}$ with a parabola, $\sigma_{N}^{2}=m \bar{v}^{2}+k \bar{v}+n$. The difference of inverse compactivity relative to that of $\bar{v}_{1}$ can be computed by the integral of fluctuationdissipation theorem:

$$
\left.\Delta \frac{1}{\chi_{F D T}}\right|_{\bar{v}, \bar{v}_{1}}=\int_{\bar{v}}^{\bar{v}_{1}} \frac{N \mathrm{~d} \bar{v}}{m \bar{v}^{2}+k \bar{v}+n}
$$


The result is plotted as a solid line in Fig. 8.5. $\left.\frac{1}{\chi_{F D T}}\right|_{1}$ is set to be 0 as well.

To quantitatively compare $\Delta \frac{1}{\chi_{O H}}$ and $\frac{1}{\chi_{F D T}}$ we computed the difference defined as:

$$
\zeta^{2}=\left(\frac{\left.\Delta \frac{1}{\chi_{O H}}\right|_{1,2}-\left.\Delta \frac{1}{\chi_{F D T}}\right|_{1,2}}{\left.\Delta \frac{1}{\chi_{O H}}\right|_{1,2}+\left.\Delta \frac{1}{\chi_{F D T}}\right|_{1,2}}\right)^{2}+\left(\frac{\left.\Delta \frac{1}{\chi_{O H}}\right|_{2,3}-\left.\Delta \frac{1}{\chi_{F D T}}\right|_{2,3}}{\left.\Delta \frac{1}{\chi_{O H}}\right|_{2,3}+\left.\Delta \frac{1}{\chi_{F D T}}\right|_{2,3}}\right)^{2}
$$

We plot $\zeta$ with cluster size $N$ in Fig. 8.6. The difference on average is below $10 \%$. 


\section{Conclusion and Outlook of Part II}

The correlation evolution with $\phi_{\text {avg }}$ in $3 \mathrm{D}$ packings is qualitatively different from that in $2 \mathrm{D}$ packings. In $3 \mathrm{D}$ packings dense packing has stronger positive correlation. This unexpected evolution of correlation can be used to explain the fluctuation of the volume versus the averaged volume. For instance, the fluctuation of the volume is reported to be a minimum at $\phi=0.596$ [Schröter et al. 2005]. Nevertheless in 2D the fluctuation decreases monotonically with packing fraction [e.g., Lechenault and Daniels [2010] and Chapter 5]. The compactivity measurement is validated for both the overlapping histogram and the fluctuation-dissipation theorem. To perform a systematic analysis of compactivity more experiments on different packing fractions need to be done.

The ageing of the experimental bed can be seen in the inset of Fig. 8.2. It is possible that the friction of beads decreases with tapping number as reported by Schröter et al. 2005. As discussed in Chapter 5 compactivity measurement depends on the friction coefficient in general. The friction also plays a role in the possible local arrangements, in other words, the fluctuation of the local volumes. Therefore the abnormal correlation feature and the compactivity measurement presented in this chapter should be checked with a reproducible fluidized bed.

The detected centroid of beads are very accurate [cf. Fig. 7.4(b) in Chapter 7]. Those data is useful to probe the phase space of local configurations, e.g., as that has been done in a frictionless 7 disc packing Gao et al., 2009]. A novel method to measure a static correlation length has been proposed [Kurchan and Levine, 2011, Sausset and Levine, 2011], based on the frequency of occurrence of "patches". The main idea is to compute the entropy of patches of a given size $R$ appearing in a system, and then to look at how this entropy scales with $R$. To compute the frequency of occurrence of patches one has to first construct the equivalence classes, in which the patches are congruent with each other. The precise centroid is helpful to finely define such patch classes.

In the dynamic experiments [cf. Chapter 6] we observed the evidence of the existence of dynamic heterogeneities in three dimensions granular system. However the exposure time of the tomograms is too long to track the fast moving particles. 


\section{Bibliography}

Aste, T. and Di Matteo, T. (2007). Correlations and aggregate statistics in granular packs. Eur. Phys. J. E 22, 235-240.

Aste, T. and Di Matteo, T. (2008). Emergence of gamma distributions in granular materials and packing models. Phys. Rev. E 77, 021309.

Aste, T., Matteo, T. D., Saadatfar, M., Senden, T. J., Schröter, M. and Swinney, H. L. (2007). An invariant distribution in static granular media. EPL 79, 24003.

Ball, R. and Blumenfeld, R. (2002). Stress Field in Granular Systems: Loop Forces and Potential Formulation. Phys. Rev. Lett. 88, 115505.

Bernal, J. D. and Mason, J. (1960). Co-ordination of Randomly Packed Spheres. Nature 188, 910.

Bertin, E., Martens, K., Dauchot, O. and Droz, M. (2007). Intensive thermodynamic parameters in nonequilibrium systems. Phys. Rev. E 75, 031120.

Blumenfeld, R. and Edwards, S. F. (2003). Granular entropy: Explicit calculations for planar assemblies. Phys. Rev. Lett. 90, 114303.

Blumenfeld, R. and Edwards, S. F. (2009). On granular stress statistics: Compactivity, angoricity, and some open issues. J. Phys. Chem. B 113, 3981-3987.

Blumenfeld, R., Jordan, J. F. and Edwards, S. F. (2012). Interdependence of the volume and stress ensembles and equipartition in statistical mechanics of granular systems. Phys. Rev. Lett. 109, 238001.

Briscoe, C., Song, C., Wang, P. and Makse, H. A. (2008). Entropy of Jammed Matter. Phys. Rev. Lett. 101, 188001.

Brown, E., Rodenberg, N., Amend, J., Mozeika, A., Steltz, E., Zakin, M. R., Lipson, H. and Jaeger, H. M. (2010). Universal robotic gripper based on the jamming of granular material. Proceedings of the National Academy of Sciences 107, 18809-18814.

Brujić, J., Wang, P., Song, C., Johnson, D., Sindt, O. and Makse, H. (2005). Granular Dynamics in Compaction and Stress Relaxation. Phys. Rev. Lett. 95, 128001.

Cates, M. E., Wittmer, J. P., Bouchaud, J.-P. and Claudin, P. (1998). Jamming, Force Chains, and Fragile matter. Phys. Rev. Lett. 81, 1841-1844.

Ciamarra, M. and Coniglio, A. (2008). Random Very Loose Packings. Phys. Rev. Lett. 101, 128001.

Ciamarra, M. P. (2007). Comment on "Granular Entropy: Explicit Calculations for Planar Assemblies". Phys. Rev. Lett. 99, 89401. 
D'Anna, G. and Gremaud, G. (2001). The jamming route to the glass state in weakly perturbed granular media. Nature 413, 407.

Dauchot, O., Marty, G. and Biroli, G. (2005). Dynamical Heterogeneity Close to the Jamming Transition in a Sheared Granular Material. Phys. Rev. Lett. 95, 265701.

Dean, D. S. and Lefèvre, A. (2003). Possible test of the thermodynamic approach to granular media. Phys. Rev. Lett. 90, 198301.

Debenedetti, P. G. and Stillinger, F. H. (2001). Supercooled liquids and the glass transition. Nature 410, 259-267.

Desmond, K. W. and Weeks, E. R. (2009). Random close packing of disks and spheres in confined geometries. Phys. Rev. E 80, 051305.

Donev, A., Stillinger, F. and Torquato, S. (2005). Unexpected Density Fluctuations in Jammed Disordered Sphere Packings. Phys. Rev. Lett. 95, 090604.

Ediger, M. (2000). Spatially heterogeneous dynamics in supercooled liquids. Ann. Rev. Phys. Chem. 51, 99-128.

Edwards, S. and Oakeshott, R. (1989). Theory of powders. Physica A 157, 10801090.

Frenkel, G., Blumenfeld, R., Grof, Z. and King, P. R. (2008). Structural characterization and statistical properties of two-dimensional granular systems. Phys. Rev. E 77,041304 .

Gabrielli, A., Joyce, M. and Sylos Labini, F. (2002). Glass-like universe: Real-space correlation properties of standard cosmological models. Phys. Rev. D 65, 083523.

Gao, G.-J., Blawzdziewicz, J., O'Hern, C. S. and Shattuck, M. (2009). Experimental demonstration of nonuniform frequency distributions of granular packings. Phys. Rev. E 80, 061304.

Goldhirsch, I. and Zanetti, G. (1993). Clustering instability in dissipative gases. Phys. Rev. Lett. 70, 1619-1622.

Gravish, N., Umbanhowar, P. B. and Goldman, D. I. (2010). Force and flow transition in plowed granular media. Phys. Rev. Lett. 105, 128301.

Henkes, S. and Chakraborty, B. (2009). Statistical mechanics framework for static granular matter. Phys. Rev. E 79, 061301.

Henkes, S., O'Hern, C. and Chakraborty, B. (2007). Entropy and Temperature of a Static Granular Assembly: An Ab Initio Approach. Phys. Rev. Lett. 99, 038002.

Hilhorst, H. J. (2008). Statistical properties of planar Voronoi tessellations. Eur. Phys. J. B 64, 437 . 
Jaynes, E. T. (1957). Information theory and statistical mechanics. Physical Review 106, 620-630.

Jin, Y. and Makse, H. a. (2010). A first-order phase transition defines the random close packing of hard spheres. Physica A 389, 5362-5379.

Kabla, A. J. and Senden, T. J. (2009). Dilatancy in slow granular flows. Phys. Rev. Lett. 102, 228301.

Kanatani, K.-I. (1981). The use of entropy in the description of granular materials. Powder Technology 30, 217.

Keys, A. S., Abate, A. R., Glotzer, S. C. and Durian, D. J. (2007). Measurement of growing dynamical length scales and prediction of the jamming transition in a granular material. Nature Physics 3, 260-264.

Komatsu, T. S., Inagaki, S., Nakagawa, N. and Nasuno, S. (2001). Creep Motion in a Granular Pile Exhibiting Steady Surface Flow. Phys. Rev. Lett. 86, 1757-1760.

Kumar, S., Kurtz, S. K. and Weaire, D. (1994). Average number of sides for the neighbours in a Poisson-Voronoi tesselation. Philos. Mag. B 69, 431-435.

Kumar, V. S. and Kumaran, V. (2005). Voronoi cell volume distribution and configurational entropy of hard-spheres. The Journal of Chemical Physics 123, 114501.

Kurchan, J. and Levine, D. (2011). Order in glassy systems. J. Phys. A 44, 035001.

Latz, A. (2000). Non-equilibrium mode-coupling theory for supercooled liquids and glasses. Journal of Physics: Condensed Matter 12, 6353-6363.

Lechenault, F., Cruz, F. d., Dauchot, O. and Bertin, E. (2006). Free volume distributions and compactivity measurement in a bidimensional granular packing. $J$. Stat. Mech. 2006, P07009-P07009.

Lechenault, F. and Daniels, K. E. (2010). Equilibration of granular subsystems. Soft Matter 6, 3074.

Lechenault, F., Dauchot, O., Biroli, G. and Bouchaud, J. P. (2008). Critical scaling and heterogeneous superdiffusion across the jamming/rigidity transition of a granular glass. EPL (Europhysics Letters) 83, 46003.

Liu, A. J. and Nagel, S. R. (1998). Jamming is not just cool any more. Nature 396, 21.

Liu, C.-h. and Nagel, S. R. (1992). Sound in Sand. Phys. Rev. Lett. 68, 2301-2305.

Liu, C.-h., Nagel, S. R., Schecter, D. A., Coppersmith, S. N., Majumdar, S., Narayan, O. and Witten, T. A. (1995). Force Fluctuations in Bead Packs. Science 169, 513 . 
Lois, G., Zhang, J., Majmudar, T. S., Henkes, S., Chakraborty, B., O'Hern, C. S. and Behringer, R. P. (2009). Stress correlations in granular materials: An entropic formulation. Phys. Rev. E 80, 060303(R).

Majmudar, T. S. and Behringer, R. P. (2005). Contact force measurements and stress-induced anisotropy in granular materials. Nature 435, 1079-1082.

Marty, G. and Dauchot, O. (2005). Subdiffusion and Cage Effect in a Sheared Granular Material. Phys. Rev. Lett. 94, 015701.

McNamara, S., Richard, P., de Richter, S. K., Le Caër, G. and Delannay, R. (2009). Measurement of granular entropy. Phys. Rev. E 80, 031301.

Mikkelsen, R., van der Meer, D., van der Weele, K. and Lohse, D. (2002). Competitive Clustering in a Bidisperse Granular Gas. Phys. Rev. Lett. 89, 214301.

Nowak, E. R., Knight, J. B., Ben-Naim, E., Jaeger, H. M. and Nagel, S. R. (1998). Density fluctuations in vibrated granular materials. Phys. Rev. E 57, 19711982.

OD́onovan, C. B. and Möbius, M. E. (2011). Spatial correlations in polydisperse, frictionless, two-dimensional packings. Phys. Rev. E 84, 020302.

Oger, L., Gervois, A., Troadec, J. P. and Rivier, N. (1996). Voronoi tessellation of packings of spheres: Topological correlation and statistics. Philos. Mag. B 74, 177-197.

O'Hern, C. S., Langer, S. A., Liu, A. J. and Nagel, S. R. (2002). Random Packings of Frictionless Particles. Phys. Rev. Lett. 88, 075507.

O'Hern, C. S., Silbert, L. E. and Nagel, S. R. (2003). Jamming at zero temperature and zero applied stress: The epitome of disorder. Phys. Rev. E 68, 011306.

Onoda, G. Y. and Liniger, E. G. (1990). Random Loose Packings of Uniform Spheres and the Dilatancy Onset. Phys. Rev. Lett. 64, 2727-2730.

Pouliquen, O., Belzons, M. and Nicolas, M. (2003). Fluctuating Particle Motion during Shear Induced Granular Compaction. Phys. Rev. Lett. 91, 014301.

Puckett, J. G. and Daniels, K. E. (2012). Equilibrating temperature-like variables in jammed granular subsystems. arXiv:1207.7349v1.

Puckett, J. G., Lechenault, F. and Daniels, K. E. (2011). Local origins of volume fraction fluctuations in dense granular materials. Phys. Rev. E 83, 041301.

Radin, C. (2008). Random Close Packing of Granular Matter. Journal of Statistical Physics 131, 567-573.

Reatto, L. and Chester, G. (1967). Phonons and the Properties of a Bose System. Physical Review 155, 88-100. 
Rycroft, C., Grest, G., Landry, J. and Bazant, M. (2006). Analysis of granular flow in a pebble-bed nuclear reactor. Phys. Rev. E 74, 021306.

Sausset, F. and Levine, D. (2011). Characterizing Order in Amorphous Systems. Phys. Rev. Lett. 107, 045501.

Schröter, M., Goldman, D. I. and Swinney, H. L. (2005). Stationary state volume fluctuations in a granular medium. Phys. Rev. E 71, 030301.

Scott, G. D. (1962). Radial distribution of the random close packing of equal spheres. Nature 194, 956.

Shahinpoor, M. (1980). Statistical Mechanical Considerations on the Random Packing of Granular Materials. Powder Technology 25, 163-176.

Silbert, L. E., Liu, A. and Nagel, S. (2005). Vibrations and Diverging Length Scales Near the Unjamming Transition. Phys. Rev. Lett. 95, 098301.

Slotterback, S., Toiya, M., Goff, L., Douglas, J. F. and Losert, W. (2008). Correlation between particle motion and Voronoi-Cell-Shape fluctuations during the compaction of granular matter. Phys. Rev. Lett. 101, 258001.

Song, C., Wang, P. and Makse, H. A. (2008). A phase diagram for jammed matter. Nature 453, 629-632.

Stoyan, D., Kendal, W. S. and Mecke, J. (1995). Stochastic Geometry and its Applications. Chichster: Wiley.

Tewari, S., Tithi, B., Ferguson, A. and Chakraborty, B. (2009). Growing length scale in gravity-driven dense granular flow. Phys. Rev. E 79, 011303.

Torquato, S., Truskett, T. and Debenedetti, P. (2000). Is random close packing of spheres well defined? Phys. Rev. Lett. 84, 2064.

Toyabe, S., Sagawa, T., Ueda, M., Muneyuki, E. and Sano, M. (2010). Experimental demonstration of information-to-energy conversion and validation of the generalized Jarzynski equality. Nature Physics 6, 988-992.

Vanel, L., Howell, D., Clark, D., Behringer, R. P. and Clément, E. (1999). Memories in sand: experimental tests of construction history on stress distributions under sandpiles. Phys. Rev. E 60, R5040.

Weele, K. V. D., Meer, D. V. D., Versluis, M. and Lohse, D. (2007). Hysteretic clustering in granular gas. EPL 53, 328-334. 


\section{Acknowledgements}

I would like to show sincere thanks to the following people:

My dear parents for raising me up and encouraging me to face difficulties in my life;

My supervisor Dr. Matthias Schröter for leading me to a new field in physics and his help with my living in Göttingen;

My examining committee: Dr. Christian Bahr, Prof. Dr. Stephan Herminghaus, Prof. Dr. Sara Köster, Prof. Brian Utter, Prof. Dr. Katharina Vollmayr-Lee and Prof. Dr. Annette Zippelius;

My kind proofreaders: Annika Döring, Julie Murison, Dr. Nirmal Thyagu Natarajan;

My collaborators: Annika Döring, Sara Gadeberg, Dr. Jean-Francois Mètayer, Julie Murison, Dr. Mario Scheel, Chih-Wei Peng for their day and night shifts in ESRF (-;

The SMGM group members for their helpful discussions and knowledge: Annika Döring, Dr. Christoph Gögelein, Dr. Jean-Francois Mètayer, Julie Murison, Dr. Nirmal Thyagu Natarajan, Max Neudecker, Welm Pätzold, Chih-Wei Peng, Dr. Sonia Utermann;

Our IT technician Thomas Eggers for his constant supporting on my computer issues;

Our machinery technician: Wolf Keiderling and Udo Krafft, and our machine shop for their brilliant work of building the experimental setup;

Prof. Dr. Karen Daniels for inviting me to visit her lab;

Finally this small beautiful city - Göttingen - I really enjoyed my life here. 


\section{Publications}

S. C. Zhao, S. Sidle, H. L. Swinney and M. Schröter, Correlation between Voronoi volumes in disc packings, EPL (Europhysics), 97, 34004

S. C. Zhao and M. Schröter, Measuring the configurational temperature of a binary disc packing, arXiv:1302.6987 